\title{
Exclusive Dealing and Vertical Integration in Interlocking Relationships*
}

\author{
Volker Nocke ${ }^{\dagger} \quad$ Patrick Rey ${ }^{\ddagger}$
}

July 18, 2017

\begin{abstract}
We develop a model of interlocking bilateral relationships between upstream firms (manufacturers) that produce differentiated goods and downstream firms (retailers) that compete imperfectly for consumers. Contract offers and acceptance decisions are private information to the contracting parties. We show that both exclusive dealing and vertical integration between a manufacturer and a retailer lead to vertical foreclosure, to the detriment of consumers and society. Finally, we show that firms have indeed an incentive to sign such contracts or to integrate vertically.
\end{abstract}

JEL Classification: L13, L42, D43.

Keywords: vertical relations, exclusive dealing, vertical merger, foreclosure, bilateral contracting.

${ }^{*}$ We thank Johannes Hörner, Robin Lee, Tore Nilssen, Markus Reisinger, Mike Riordan, Jean Tirole, Mike Whinston, Thibaud Vergé, and Ali Yurukoglu for their comments; we also thank participants at the SEARLE 2012 Antitrust Symposium, the Cowles 2012 Economic Theory conference, the BECCLE 2012 conference, the EARIE 2013 conference, the Toulouse 2012 Workshop on Market Power in Vertically Related Industries, the Toulouse 2013 TRANSFOP conference, and the 2016 MaCCI Summer Institute in Competition Policy, as well as at seminars at Harvard, Bocconi (Milan), X-CREST (Paris), Virginia, CERGE-EI (Prague), IESE (Barcelona), LMU Munich and Western Ontario. Financial support from the German Science Foundation (DFG) and the European Research Council (ERC) under the European Community's Seventh Framework Programme (FP7/2007-2013) Grant Agreement $\mathrm{N}^{\circ} \mathrm{s} 313623$ and 340903 is gratefully acknowledged.

${ }^{\dagger}$ University of California, Los Angeles. Email: volker.nocke@gmail.com.

‡Toulouse School of Economics, University of Toulouse Capitole. Email: patrick.rey@tse-fr.eu. 


\section{Introduction}

In this paper, we study "interlocking relationships" in vertically related oligopolies, where the same competing upstream firms deal with the same competing downstream firms. We develop a framework that allows for general contracts between upstream and downstream firms, the terms of which are private information to the contracting parties. In this framework, firms have an incentive to sign exclusive dealing provisions or, alternatively, to integrate vertically, at the expense of consumers and society. The contribution of this paper is thus two-fold: It provides a general and yet tractable framework for the analysis of interlocking relationships; and it sheds light on the long-standing policy debate on vertical foreclosure.

Interlocking relationships. In many if not most consumer goods markets, competing retailers carry the products of the same competing national brand manufacturers. For example, most supermarkets carry both Coca Cola and Pepsi Cola, and pay-TV operators offer access to the channels of the same content providers. ${ }^{1}$ Such interlocking relationships are also endemic in intermediate goods markets. For example, Airbus and Boeing procure components (e.g., avionics, wheels and brakes) from the same competing suppliers (such as Honeywell and Thales); and insurance companies often deal with the same health care service providers. ${ }^{2}$ Yet, despite their prevalence in practice, there is little work on interlocking relationships in the literature. ${ }^{3}$ One reason for this is that these relations are fraught with contracting externalities and therefore difficult to analyze. Indeed, the gains from trade that two firms can achieve together depend not only on the agreement that they can reach, but also on the agreements that each can sign with other partners, as well as on the agreements that these other partners can sign with each other. In this paper, we develop a framework that fully accounts for these externalities and yet provides clear predictions about likely outcomes, in terms of prices and outputs, distribution of profits, and firms' incentives to opt for exclusivity or vertical integration.

Vertical foreclosure. It is widely recognized that exclusive dealing and vertical integration can be motivated by efficiency considerations. Whether firms can also engage in exclusive dealing or merge vertically for anti-competitive purposes has been the object of a long-standing debate

\footnotetext{
${ }^{1}$ For an analysis of vertical relations in the U.S. cable TV industry, see, e.g., Chipty and Snyder (1999), Crawford and Yurukoglu (2012), and Crawford, Lee, Whinston, and Yurukoglu (2015).

${ }^{2}$ For recent empirical studies of U.S. private health care markets, see, e.g., Gowrisankaran, Nevo, and Town (2015) and Ho and Lee (2015).

${ }^{3}$ We discuss this literature, and how our paper relates to it, in Section 7.
} 
in policy circles as well as in the academic literature. ${ }^{4}$ The so-called Chicago critique pointed out that the "monopoly leverage" concept originally used was based on a confusion, as there is only one source of profit in a vertically related industry. In response to this critique, Ordover, Saloner and Salop $(1990)^{5}$ showed that an integrated firm may stop supplying downstream rivals, in order to confer market power to the other suppliers and raise in this way downstream rivals' costs. As noted by Hart and Tirole (1990) and Reiffen (1992), however, this analysis relied on the assumptions that (i) an integrated supplier could somehow pre-commit itself not to supply downstream rivals (as ex post it would have an incentive to supply the rival), and (ii) contracting with the other suppliers is inefficient (linear tariffs, giving rise to double marginalization). Hart and Tirole (1990), O'Brien and Shaffer (1992), and McAfee and Schwartz (1994) emphasize instead that, under secret contracting, exclusive dealing or vertical integration can help a dominant supplier exert its market power. While these papers are not subject to the same limitations as Ordover, Salop and Saloner (1990), they restrict attention to upstream monopoly or quasi-monopoly settings, which has severely limited their impact on actual policy decisions. We contribute to this debate by showing the robustness of these previous insights to more complex environments, which is important for competition authorities: not only in quasimonopoly settings but also in interlocking relationships with differentiated products, firms can have incentives to engage in vertical foreclosure in order to exert market power at the expense of consumers and society. This is not to say that exclusive dealing or vertical integration is necessarily bad for consumers or society. For the sake of exposition, we focus here on strategic effects, and abstract from well-known potential efficiencies; in practice, competition agencies may have to balance the pro- and anticompetitive effects of these agreements.

Framework. We develop a rather general and yet tractable framework for the analysis of multilateral vertical relationships. In particular, this framework allows for general (menus of) non-linear tariffs, fairly general demands for suppliers' goods, and arbitrary bargaining power between upstream and downstream firms. A key ingredient is secret contracting: contracting terms and acceptance decisions are assumed to be private information to the contracting parties. We believe that this is a plausible assumption for many industries, in which firms are actually keen to protect the confidentiality of their agreements. ${ }^{6}$ This assumption also allows

\footnotetext{
${ }^{4}$ For reviews of this debate, see, e.g., Rey and Tirole (2007) and Whinston (2006).

${ }^{5}$ See also Salinger (1988).

${ }^{6}$ See Smith and Thanassoulis (2015) for evidence that supermarkets keep the contracts with their suppliers secret.
} 
us to abstract from strategic pre-commitment effects, in which firms distort the terms of their agreements in order to influence the behavior of their rivals. ${ }^{7}$

Contractual arrangements between upstream and downstream firms necessarily involve externalities. Analyzing multilateral bargaining with externalities is complex, and the theory literature has arguably not yet provided a widely accepted general solution. In light of this, and for tractability, we consider a bargaining process in which, with some probability, either the upstream firms get to make take-it-or-leave-it offers to their downstream partners, or the downstream firms are the ones to make the offers.

Modelling secret contracting raises issues about firms' anticipations about other firms' contracting arrangements. In particular, when receiving an unexpected offer from a trading partner, a firm must form beliefs about the contracts that the partner is negotiating with the firm's rivals. The literature has mostly focused on simple or reasonable beliefs such as passive beliefs, where recipients of unexpected offers do not revise their beliefs about other firms' contracts, and wary beliefs, where the receiver of a deviant offer assumes that the proposer offers other partners the best contract (from the proposer's standpoint), given the contract offered to the receiver. The complexity of the issues raised by these out-of-equilibrium beliefs depends on the nature of competition. For instance, even in the simple case where a single firm is present in the upstream market, it is known that passive beliefs give rise to existence problems when the upstream firm makes the offers and downstream firms compete in prices, and wary beliefs are moreover not very tractable in that case. ${ }^{8}$ By contrast, when downstream firms compete in quantities, wary beliefs coincide with passive beliefs, and the analysis is also much more tractable. ${ }^{9}$ In the light of these observations, in order to provide a general and yet tractable framework, it seems natural to start with the case of Cournot competition among downstream firms. This is the route taken in this paper.

Insights. We consider a successive duopoly setting in which upstream firms supply differentiated goods to downstream Cournot competitors. We provide mild regularity conditions ensuring that, in the absence of any exclusive dealing or vertical integration, there exists a unique equilibrium outcome in terms of downstream prices and quantities, which is independent of firms' bargaining power. By contrast, the division of profit depends not only on bargaining power

\footnotetext{
${ }^{7}$ These strategic effects have for instance been analyzed by Bonanno and Vickers (1988), Rey and Stiglitz (1988, 1995), Gal-Or (1991). For a review of this literature, see, e.g., Caillaud and Rey (1995).

${ }^{8}$ See Rey and Vergé (2004).

${ }^{9}$ See Hart and Tirole (1990).
} 
but also on the types of contracts signed in equilibrium. Upstream firms prefer the equilibrium outcome induced by two-part tariffs - which is also the unique equilibrium outcome when belowcost pricing is ruled out - but downstream firms can obtain a larger share of the profit - and can even appropriate the entire profit - with other non-linear contracts.

We also characterize the equilibria that arise under exclusive dealing and vertical integration. Furthermore, we show that, regardless of firms' relative bargaining power, and regardless of which equilibrium is selected in the various situations, firms have an incentive to adopt exclusive distribution provisions or to integrate vertically. By contrast, other exclusive deal arrangements, such as single branding, are less attractive to the firms. Finally, we show that exclusive dealing and vertical integration harm consumers and society.

Roadmap. Section 2 describes our framework. Section 3 establishes that contracts signed by independent suppliers are bilaterally efficient. Section 4 provides a complete characterization of equilibria in the absence of exclusive dealing and vertical integration. Sections 5 and 6 , respectively, study exclusive dealing and vertical integration. Section 7 relates our paper to the literature. Section 8 concludes.

\section{The Framework}

We consider a successive duopoly framework. For the sake of exposition, we will refer to the upstream firms as manufacturers and to the downstream firms as retailers; it should however be clear that the analysis can be transposed to other types of vertically related industries. Specifically, two differentiated manufacturers, $M_{A}$ and $M_{B}$, distribute their goods through two retailers, $R_{1}$ and $R_{2}$, who compete à la Cournot in the downstream market. To focus on vertical agreements, we rule out any kind of "horizontal" mechanisms such as, e.g., loyalty rebates or Most Favored Nation provisions, ${ }^{10}$ and consider instead contracts purely based on the quantity traded: Formally, a contract between $M_{i}$ and $R_{h}$ is a tariff $\tau_{i h}: \Re_{+} \rightarrow \Re$, where $\tau_{i h}(q)$ is the payment from $R_{h}$ to $M_{i}$ in return for a quantity $q$ of good $i{ }^{11}$ We do not impose any further

\footnotetext{
10 "Loyalty rebates" are discounts based on market shares (e.g., conditional on the customer making at least x\% of its purchase from a given supplier). European antitrust authorities treat them as anti-competitive when they are used by dominant suppliers. "Most Favored Nation" clauses require the supplier to grant the customer the best conditions made to any other customer. They, too, have been subject to scrutiny by antitrust authorities.

${ }^{11}$ For the sake of exposition, we will assume that parties contract on the quantity $q$ sold to consumers, rather than the quantity bought from the manfacturer. The distinction becomes moot when the production cost is large enough, as then a retailer will not want to buy more than it needs in any relevant scenario.
} 
restriction, however, and thus allow for any nonlinear tariff; special cases of interest are:

- Two-part tariff: $\tau_{i h}(q)=F+w q$, where $F$ is the fixed (or "franchise") fee, and $w \geq 0$ the marginal wholesale price; we will denote such a two-part tariff by $(w, F)$.

- Forcing contract:

$$
\tau_{i h}(q)=\left\{\begin{array}{cc}
\hat{T} & \text { if } q=\hat{q} \\
\infty & \text { otherwise }
\end{array}\right.
$$

where $\hat{q}$ is the "forced" quantity; we will denote such a forcing contract by $(\hat{q}, \hat{T})$.

As discussed in the introduction, we assume that firms engage in secret bilateral negotiations. Furthermore, to allow for balanced bargaining power, we assume further that both sides (manufacturers or retailers) get to make take-it-or-leave-it offers with some probability. To maintain symmetry in the resulting principal-agent relationship, we suppose that the receiving side then picks the quantity to be traded.

The timing is as follows:

Stage 1 With probability $\alpha$ (resp., $1-\alpha$ ), manufacturers (resp., retailers) simultaneously offer (secret) contracts to the retailers (resp., manufacturers).

Stage 2 The recipients of the offers simultaneously (and secretly): (i) accept or reject the offers; and (ii) for each accepted contract, choose how much to put on the final market. ${ }^{12}$ The resulting prices are such that markets clear.

As is well-known, this type of game has many Perfect Bayesian Equilibria. This is because, when a firm receives an unexpected (i.e., off-equilibrium) offer, there is considerable leeway in how it might revise its beliefs about the offer made to its rival, and thus in how it will react to the deviant offer. As argued by McAfee and Schwartz (1994), it is however natural to assume that firms interpret any offer made as a deliberate choice by the proposer. This leads to focus on "wary beliefs", where the receiver of an unexpected offer thinks that the offer made to its rival is the proposer's optimal choice, given the offer just received.

Consider first the case when manufacturers are the proposers. From the point of view of a manufacturer, the two retailers form two completely separate markets - in particular, because

\footnotetext{
${ }^{12}$ As acceptance and output decisions are simultaneous, there is no role here for menus of contracts: Offering a menu of tariffs is de facto equivalent to offering the envelope of these tariffs (the lower envelope if manufacturers are the proposers, or the upper one, if retailers are the proposers). It will however be sometimes convenient to refer to menus of forcing contracts.
} 
contract offers are secret, each retailer's willingness to pay does not depend on the contract actually offered to its rival. Thus a manufacturer has no incentive to change its offer to one retailer when it alters the other retailer's contract. As a result, wary beliefs coincide with so-called "passive" or "market-by-market-bargaining" conjectures: retailers do not revise their expectations about the other contracts when they receive a deviant offer.

Consider now the case when retailers are the proposers. As the manufacturers' goods are (possibly imperfect) substitutes, the quantity that a retailer wishes to trade with one manufacturer typically depends on the quantity traded with the other manufacturer. Hence, a manufacturer should anticipate that, if a retailer offers it an out-of-equilibrium contract, the latter has an incentive to change the terms offered to the other manufacturer. However, for an independent manufacturer, the profit obtained by dealing with one retailer does not depend on the terms of trade with the other retailer, and thus the issue of out-of-equilibrium beliefs becomes moot. ${ }^{13}$ By contrast, an integrated manufacturer worries about the impact on its downstream subsidiary, and as a result wary beliefs no longer coincide with passive beliefs, as the quantity that a retailer wishes to trade with one manufacturer typically depends on the quantity traded with the other manufacturer.

We will consider variants of the above baseline model in which the firms can either engage in exclusive dealing or vertical integration. An exclusive dealing provision restricts the set of partners to whom offers can be made or from whom they can be accepted. In case $M_{i}$ and $R_{h}$ are vertically integrated, we assume that they maximize their joint profits, regardless of internal transfer prices; in addition, $M_{i}$ and $R_{h}$ "share information" in the sense that, when making an acceptance or output decision, the integrated firm knows the terms of the offer made by its own subsidiary to the rival. ${ }^{14}$

For expositional simplicity, we assume that manufacturers are symmetric and that retailers are perfect substitutes. Specifically, each manufacturer $M_{i}$, for $i \in\{A, B\}$, produces good $i$

\footnotetext{
${ }^{13}$ When the manufacturer is indifferent between supplying different quantities to a given retailer, this choice could in theory depend on the contract offered by the other retailer. This, in turn, could be used to "punish" deviations by the other retailer. We will discard such possibility and assume that the quantity supplied by the manufacturer to a given retailer depends only on the agreement reached with that retailer. [Introducing arbitrarily small shocks to manufacturers' costs, not observed by retailers, would suffice to solve this issue, as manufacturers could no longer be made indifferent between alternative options.]

${ }^{14}$ As we assume that acceptance and output decisions are made simultaneously, we do not need to take a stance on how an integrated firm would interpret an unexpected acceptance or rejection of the offer made by its own subsidiary.
} 
at the same constant unit $\operatorname{cost} c>0$, and the two retailers face the same constant unit cost, which for convenience we normalize to zero. The inverse demand for good $i=A, B$ is given by $P\left(Q_{i}, Q_{j}\right), j \neq i \in\{A, B\}$, where $Q_{i} \equiv q_{i 1}+q_{i 2}$ denotes total consumption of good $i$, and $q_{i h} \geq 0$ the quantity of good $i$ purchased from retailer $R_{h}, h \in\{1,2\}$. Total industry profit is therefore given by:

$$
\Pi\left(Q_{A}, Q_{B}\right) \equiv\left[P\left(Q_{A}, Q_{B}\right)-c\right] Q_{A}+\left[P\left(Q_{B}, Q_{A}\right)-c\right] Q_{B},
$$

and the profit generated by $R_{h}$ can be expressed as, for $h \neq k \in\{1,2\}$ :

$$
\Pi_{h}\left(q_{A h}, q_{B h} ; q_{A k}, q_{B k}\right) \equiv\left[P\left(Q_{A}, Q_{B}\right)-c\right] q_{A h}+\left[P\left(Q_{B}, Q_{A}\right)-c\right] q_{B h} .
$$

Throughout the paper, we assume that goods $A$ and $B$ are (imperfect) substitutes, and that there is a viable demand for each unless the other one floods the market:

(A.1) For any $\left(Q_{A}, Q_{B}\right) \geq 0,{ }^{15}$

$$
\partial_{1} P\left(Q_{A}, Q_{B}\right) \leq \partial_{2} P\left(Q_{A}, Q_{B}\right) \leq 0
$$

with strict inequalities when $P\left(Q_{A}, Q_{B}\right)>0$.

(A.2) $P(0,0)>c$ and, for $Q$ sufficiently large, $P(0, Q)<c$.

\section{Cost-based Contracts}

In this section, we define the notion of a "cost-based" contract, in which the marginal input price coincides with the marginal cost of production, and show that unintegrated manufacturers sign cost-based contracts with every available retailer.

Throughout the paper, we will use indices $i \neq j$ when referring to $M_{A}$ and $M_{B}$, and $h \neq$ $k$ when referring to $R_{1}$ and $R_{2}$. Let

$$
\chi\left(q_{i k}, q_{j h}, q_{j k}\right) \equiv \arg \max _{q_{i h}}\left[P\left(q_{i 1}+q_{i 2}, q_{j 1}+q_{j 2}\right)-c\right] q_{i h}+P\left(q_{j 1}+q_{j 2}, q_{i 1}+q_{i 2}\right) q_{j h}
$$

denote the set of bilaterally efficient values for the output $q_{i h}$, from the standpoint of the pair $M_{i}-R_{h}$, holding fixed all other outputs. ${ }^{16}$ We will say that the equilibrium contract signed by $M_{i}$ and $R_{h}$ is "cost-based" if it induces a bilaterally efficient output, given the other outputs:

\footnotetext{
${ }^{15}$ Throughout the paper, $\partial_{n} f$ denotes the partial derivative of the function $f$ with respect to its $n^{\text {th }}$ argument; likewise, $\partial_{n m}^{2} f$ will denote the second-order partial derivative with respect to the $n^{\text {th }}$ and $m^{\text {th }}$ arguments.

${ }^{16}$ In the above equation, the right-hand side constitutes the joint profit of $M_{i}$ and $R_{h}$, gross of $M_{i}$ 's profit from dealing with $R_{k}, \tau_{i k}\left(q_{i k}\right)-c q_{i k}$, and of $R_{h}$ 's payment for $q_{j h}, \tau_{j h}\left(q_{j h}\right)$.
} 
Definition 1 Given the outputs of the other channels $\left(q_{i k}, q_{j h}, q_{j h}\right)$, a contract $\tau_{i h}(\cdot)$ between $M_{i}$ and $R_{h}$ is said to be cost-based if, when accepted, it induces a quantity $q_{i h} \in \chi\left(q_{i k}, q_{j h}, q_{j h}\right)$.

The following lemma shows that in equilibrium, unintegrated manufacturers sign cost-based contracts:

Lemma 1 Suppose $M_{i}$ is not vertically integrated (whereas $M_{j}$ may or may not be vertically integrated). Then, in any equilibrium $M_{i}$ signs a "cost-based" contract (as defined above) with every retailer $R_{h}$ that is available, ${ }^{17}$ given the exclusive dealing provisions (with the convention that they sign a "null" contract if it is bilaterally efficient not to trade). In addition, when the retailers are the proposers, then $M_{i}$ supplies the equilibrium quantities at cost (that is, $M_{i}$ obtains zero profit).

\section{Proof. See Appendix A.}

The intuition is the following. When the retailers are the proposers, an independent $M_{i}$ is actually willing to supply any retailer with any quantity in return for any payment covering its cost; as a result, retailers ask for the bilaterally efficient quantity, and just cover the cost. When instead the manufacturers are the proposers, the contract that an independent $M_{i}$ signs with one retailer does not affect the profit that $M_{i}$ can make with the other retailer. As a result, wary beliefs boil down to passive beliefs: Each $R_{h}$ expects its rival $R_{k}$ to stick to the equilibrium quantities even when receiving a deviant offer from $M_{i}$. As the deviant offer does not affect the profit that $M_{i}$ makes on its contract with $R_{k}$, the equilibrium contract between $M_{i}$ and $R_{h}$ must therefore maximize the joint bilateral profit of the contracting parties, given $R_{k}$ 's equilibrium quantities, which is achieved by signing a cost-based contract. Note that this logic holds regardless of whether the other contracts are cost-based or not.

As we show below, an important implication of Lemma 1 is that the equilibria replicate the outcome of differentiated-goods Cournot duopolies. Specifically, consider the following settings, in which two firms can produce goods $A$ and/or $B$ at the same constant unit cost $c$ :

- Multiproduct-firm Cournot duopoly: Both firms can produce goods $A$ and $B$.

- Asymmetric Cournot duopoly: One firm can produce both goods, whereas the other firm can produce only one good.

\footnotetext{
${ }^{17}$ That is, the contract between $M_{i}$ and $R_{h}$ induces the bilaterally efficient output $q_{i h}$, given the equilibrium outputs of the other channels.
} 
- Differentiated monoproduct-firm Cournot duopoly: One firm can produce good $A$ and the other can produce $B$.

For the sake of exposition, we will assume that these duopoly settings yield a well-behaved outcome, namely:

(P.1) In each of these settings, there is a unique equilibrium, in which all quantities are positive; in addition, whenever a firm sells both goods, the goods are "internal" strategic substitutes. ${ }^{18}$

Property (P.1) ensures that the equilibrium outcomes vary across the different duopoly settings. In particular, a firm sells every good that it can produce but, holding fixed the rival's outputs, wants to sell less of a good the more it produces of the other one. Property (P.1) holds, for instance, when demand is linear; in the Online Appendix A, we provide more general conditions on demand ensuring that (P.1) holds. In what follows, we assume that (P.1) holds.

In the multiproduct-firm Cournot duopoly, firms are symmetric; the uniqueness of the equilibrium thus implies that it is symmetric: each firm sells a quantity $q^{\circ}>0$ of each good, where $q^{\circ}$ is such that

$$
q^{\circ} \in \arg \max _{q}\left[P\left(q+q^{\circ}, 2 q^{\circ}\right)-c\right] q+\left[P\left(2 q^{\circ}, q+q^{\circ}\right)-c\right] q^{\circ}
$$

Total industry profit is positive ${ }^{19}$ and equal to

$$
\Pi^{\circ} \equiv \Pi\left(2 q^{\circ}, 2 q^{\circ}\right)=\left[P\left(2 q^{\circ}, 2 q^{\circ}\right)-c\right] 4 q^{\circ}>0 .
$$

Consider now an asymmetric Cournot duopoly where firm 1, say, offers both goods $A$ and $B$, whereas firm 2 offers good $B$ only. The equilibrium quantities are then such that

$$
\begin{aligned}
\left(q_{A 1}^{*}, q_{B 1}^{*}\right) & \in \arg \max _{q_{A 1}, q_{B 1}}\left[P\left(q_{A 1}, q_{B 1}+q_{B 2}^{*}\right)-c\right] q_{A 1}+\left[P\left(q_{B 1}+q_{B 2}^{*}, q_{A 1}\right)-c\right] q_{B 1}, \\
q_{B 2}^{*} & \in \arg \max _{q_{B 2}}\left[P\left(q_{B 1}^{*}+q_{B 2}, q_{A 1}^{*}\right)-c\right] q_{B 2} .
\end{aligned}
$$

\footnotetext{
${ }^{18}$ That is, the profit $\pi$ of a firm, as a function of its quantities $q_{A}$ and $q_{B}$, is such that $\partial^{2} \pi / \partial q_{A} \partial q_{B} \leq 0$, with a strict inequality whenever the price of at least one good is positive.

${ }^{19} \mathrm{By}$ construction, equilibrium profits cannot be negative. Furthermore, starting from a candidate equilibrium in which profits would be zero, each firm would benefit from slightly reducing any of its outputs. A similar argument applies to the other duopoly settings - in the asymmetric case, it can further be shown that both prices must be above costs.
} 
Total industry profit is thus equal to $\Pi^{*}=\Pi_{1}^{*}+\Pi_{2}^{*}$, where $\left(\right.$ with $Q_{A}^{*}=q_{A 1}^{*}$ and $Q_{B}^{*}=q_{B 1}^{*}+q_{B 2}^{*}$ )

$$
\Pi_{1}^{*}=\left[P\left(Q_{A}^{*}, Q_{B}^{*}\right)-c\right] q_{A 1}^{*}+\left[P\left(Q_{B}^{*}, Q_{A}^{*}\right)-c\right] q_{B 1}^{*}>0 \text { and } \Pi_{2}^{*}=\left[P\left(Q_{B}^{*}, Q_{A}^{*}\right)-c\right] q_{B 2}^{*}>0 .
$$

Finally, in the differentiated monoproduct-firm Cournot duopoly, firms are again symmetric and thus the uniqueness of the equilibrium implies that it is symmetric: each firm sells a quantity $Q^{* *}$ of its good, where $Q^{* *}>0$ is the unique solution to

$$
Q^{* *} \in \arg \max _{Q}\left[P\left(Q, Q^{* *}\right)-c\right] Q
$$

Total industry profit is equal to

$$
\Pi^{* *}=\Pi\left(Q^{* *}, Q^{* *}\right)=\left[P\left(Q^{* *}, Q^{* *}\right)-c\right] 2 Q^{* *}>0 .
$$

\section{Non-Exclusive Relationships and Vertical Separation}

We now characterize the equilibria in the absence of exclusive dealing and vertical integration. The following proposition shows that all equilibria entail the same downstream prices and quantities - and thus generate the same industry profit; however, they can differ in the way firms share this profit - manufacturers cannot get more, but may get less than their contribution to this industry profit:

Proposition 1 In the absence of exclusive dealing and vertical integration, the set of equilibria is non-empty and characterized as follows:

(i) The quantities $\left(q_{i h}^{\circ}\right)_{i=A, B, h=1,2}$ are the same as in the multiproduct-firm Cournot duopoly: $q_{i h}^{\circ}=q^{\circ}$.

(ii) The range of expected profits for the two manufacturers is $\left[0,2 \alpha \Delta^{\circ}\right] \times\left[0,2 \alpha \Delta^{\circ}\right]$, where

$$
\Delta^{\circ} \equiv \frac{\Pi^{\circ}}{2}-\max _{q}\left\{\left[P\left(q^{\circ}+q, q^{\circ}\right)-c\right] q\right\}
$$

denotes the manufacturer's contribution to the profit $\Pi^{\circ} / 2$ generated by a retailer.

Proof. See Appendix B.

The intuition for the first part is simple. From Lemma 1, each $M_{i}$ must sign a cost-based contract with each $R_{h}$. This, in turn, implies that the equilibrium replicates the outcome of a multiproduct-firm Cournot duopoly. 
All equilibria thus generate the same industry-wide aggregate profit, $\Pi^{\circ}$. Furthermore, when the retailers are the proposers, then from Lemma 1 manufacturers supply at cost, and thus the equilibrium outcome is unique: manufacturers obtain zero profit, and each retailer appropriates the profit that it generates. In Appendix B, it is moreover shown that this outcome can be supported in various ways. For instance, retailers could offer to buy any quantity at cost; alternatively, each retailer could offer each manufacturer a single forcing contract $\left(q^{\circ}, c q^{\circ}\right)$.

When instead the manufacturers are the proposers, every equilibrium tariff must offer more flexibility than a single forcing contract. The intuition is as follows. In equilibrium, each retailer must be indifferent between accepting both manufacturers' offers, or only one (either one): If a retailer strictly preferred dealing with both manufacturers than with only one of them, then the rival manufacturer could profitably deviate by asking for a larger share of the profits. But if, say, $M_{i}$ offers $R_{h}$ a single forcing contract $\left(q^{\circ}, T_{i h}^{\circ}\right)$, then $M_{i}$ is also indifferent between whether or not $R_{h}$ also accepts upstream rival $M_{j}$ 's offer; hence, the joint profit of $M_{i}$ and $R_{h}$ must be the same as what they would obtain if $R_{h}$ were to deal exclusively with $M_{i}$, namely, $P\left(2 q^{\circ}, q^{\circ}\right)-T_{i h}^{\circ}$. Hence, in equilibrium the sum of $R_{h}$ 's profit and of $M_{i}$ 's profit from its contract with $R_{h}$ is given by

$$
\left(T_{i h}^{\circ}-c q^{\circ}\right)+\left(P\left(2 q^{\circ}, q^{\circ}\right)-T_{i h}^{\circ}\right)=\left[P\left(2 q^{\circ}, q^{\circ}\right)-c\right] q^{\circ} .
$$

It follows that, under exclusivity, $M_{i}$ and $R_{h}$ could generate more profit by replacing $q^{\circ}$ with

$$
\hat{q} \equiv \arg \max _{q}\left\{\left[P\left(q^{\circ}+q, q^{\circ}\right)-c\right] q\right\}
$$

and share the profit increase through an appropriate transfer $\hat{T}$; that is, $M_{i}$ could profitably deviate by offering the forcing contract $(\hat{q}, \hat{T})$, thereby inducing $R_{h}$ to "bump" the rival manufacturer (note that this deviation does not affect the profit that $M_{i}$ obtains from dealing with the other retailer, $\left.R_{k}\right){ }^{20}$

Two-part tariffs, for instance, offer the desired flexibility, and they can be used to support an equilibrium in which manufacturers obtain their full contribution to industry profits, $\Delta^{\circ}$. To see why, note first that cost-based two-part tariffs allow retailers to buy any marginal quantity at cost. It follows that: (i) as a manufacturer does not care about the level of trade chosen by

\footnotetext{
${ }^{20}$ A similar bumping problem arises in a setting considered by Marx and Shaffer (2007), where two retailers are each offering a single (public) contract to a common manufacturer. As a result, the weaker retailer is excluded in equilibrium. Allowing retailers to offer menus of contracts (one explicitly or even implicitly designed for exclusivity, and one designed for non-exclusivity), restores the existence of an equilibrium where both channels are active; see Miklós-Thal et al. (2011) and Rey and Whinston (2013).
} 
retailers, it is indifferent as to whether retailers will deal with the rival manufacturer or not; and (ii) in both instances, the tariff is bilaterally efficient, that is, it maximizes the joint profit of the manufacturer and the retailer. Second, setting the equilibrium fixed fees to $\Delta^{\circ}$ makes each retailer indifferent between dealing with both manufacturers, or with either one on an exclusivity basis. It follows that manufacturers cannot profitably deviate, as they cannot increase the joint profit achieved with a retailer (regardless of whether the deviant offer induces exclusivity or not) and cannot decrease the profit of the retailer (who can always obtain its equilibrium profit by dealing exclusively with the rival).

The first part of Proposition 1 implies that the equilibrium industry profit is also the same as in the multiproduct-firm Cournot duopoly: each retailer generates a profit equal to $\Pi^{\circ}$. Furthermore, even when it can make take-it-or-leave-it offers, a manufacturer cannot ask for more than its contribution $\Delta^{\circ}$ to this profit in equilibrium, otherwise its rival could profitably deviate by inducing the retailer to opt for exclusivity. However, as is usual in these common agency situations, the more generous a manufacturer is, the more generous the other must be; as a result, there are infinitely many equilibria, in which manufacturers obtain less than their contribution to the industry profit; in particular, there are equilibria in which retailers appropriate all profits.

Offering a pair of forcing contracts suffices to provide the flexibility needed to sustain any of these equilibrium outcomes: it is shown in Appendix B that, without loss of generality, we can indeed restrict attention to equilibria in which each $M_{i}$ offers each $R_{h}$ two forcing contracts: a cost-based contract $\left(q^{\circ}, T_{i h}^{\circ}\right)$ "designed" for common agency, which $R_{h}$ accepts along the equilibrium path, and a contract $\left(\tilde{q}_{i h}^{\circ}, \tilde{T}_{i h}^{\circ}\right)$ "designed" for exclusivity, where $\tilde{q}_{i h}^{\circ}>q^{\circ}$ and $c\left(\tilde{q}_{i h}^{\circ}-q^{\circ}\right) \geq \tilde{T}_{i h}^{\circ}-T_{i h}^{\circ}>0$. Even though they are not accepted in equilibrium, the "exclusive deal" offers determine retailers' outside options, and thus how much profit is left for the manufacturers: in equilibrium, each retailer is indifferent between picking both $\left(q^{\circ}, T_{A h}^{\circ}\right)$ and $\left(q^{\circ}, T_{B h}^{\circ}\right)$, or picking only $\left(\tilde{q}_{i h}^{\circ}, \tilde{T}_{i h}^{\circ}\right)$, from either manufacturer $i=A, B$. Moreover, each manufacturer must (weakly) prefer that the retailer does not choose the exclusive deal offer but rather the one designed for common agency: if a manufacturer were to prefer the retailer to accept the exclusive deal option over the common agency option, then the manufacturer could profitably deviate by making the exclusive deal option slightly more attractive, thereby inducing the retailer to pick that option. By contrast, it may be the case that a manufacturer would be strictly worse-off if a retailer were to select it on an exclusive basis; indeed, to sustain an equilibrium that gives some manufacturer $M_{i}$ less than its contribution $\Delta^{\circ}$ to the profit generated by some retailer $R_{h}$, the 
other manufacturer, $M_{j}$, must price the incremental quantity $\tilde{q}_{j h}^{\circ}-q^{\circ}$ below cost. $^{21}$

\section{Exclusive Dealing}

In this section, we analyze the effects of exclusive dealing provisions on the equilibrium outcome and on welfare. These provisions include exclusive distribution contracts, which preclude the manufacturer from selling to the rival retailer, and single branding contracts, which preclude the retailer from buying from the other manufacturer. We first consider the case where an exclusive dealing provision precludes trade between, say, $M_{A}$ and $R_{2}$, and then the case where two exclusive dealing provisions preclude trade, e.g., between $M_{A}$ and $R_{2}$ and between $M_{B}$ and $R_{1}$. We then study firms' incentives to engage in exclusive dealing and finally consider the welfare implications.

\subsection{Equilibrium Outcomes}

- Single exclusivity. We begin by analyzing the equilibrium effects of a pre-existing exclusion dealing provision that precludes trade between, say, $M_{A}$ and $R_{2}$. Such a provision could, for instance, be an exclusive distribution contract between $M_{A}$ and $R_{1}$, or a single branding contract between $M_{B}$ and $R_{2}$. We have:

Proposition 2 Suppose that a single exclusive dealing provision precludes trade between, say, $M_{A}$ and $R_{2}$ (i.e., $\left.q_{A 2}^{*}=0\right)$. The set of equilibria is then non-empty and characterized as follows:

(i) The quantities $\left(q_{A 1}^{*}, q_{B 1}^{*}, q_{B 2}^{*}\right)$ are the same as in the corresponding asymmetric Cournot duopoly.

(ii) The range of expected profits for $M_{A}$ and $M_{B}$ is $\left[0, \alpha \Delta_{A, 1}^{*}\right] \times\left[\alpha \Pi_{2}^{*}, \alpha\left(\Pi_{2}^{*}+\Delta_{B, 1}^{*}\right)\right]$, where:

$$
\Delta_{A, 1}^{*}=\Pi_{1}^{*}-\max _{q_{B 1}}\left\{\left[P\left(q_{B 1}+q_{B 2}^{*}, 0\right)-c\right] q_{B 1}\right\},
$$

and

$$
\Delta_{B, 1}^{*}=\Pi_{1}^{*}-\max _{q_{A 1}}\left\{\left[P\left(q_{A 1}, q_{B 2}^{*}\right)-c\right] q_{A 1}\right\}
$$

respectively denote $M_{A}$ 's and $M_{B}$ 's contributions to the profit generated by $R_{1}$.

${ }^{21} \mathrm{By}$ contrast, the equilibrium based on two-part tariffs, which gives both manufacturers their contribution $\Delta^{\circ}$, is by construction such that each $M_{i}$ is indifferent between $R_{h}$ buying $q^{\circ}$ from both manufacturers, or $\tilde{q}_{i h}^{\circ}$ exclusively from $M_{i}$. This equilibrium is therefore "truthful" in the spirit of Bernheim and Whinston (1986a). 
Proof. See Appendix C.

The first part of Proposition 2 follows from Lemma 1: As contracts must be cost-based, the market outcome is the same as in an asymmetric duopoly in which one firm offers both goods $A$ and $B$, whereas the other offers only one of these goods. The second part of Proposition 2 follows the same logic as that of Proposition 1. In particular, when retailers are the proposers, they buy the bilaterally efficient quantity at cost. When instead the manufacturers are the proposers, $R_{1}$ is again indifferent between accepting both manufacturers' offers, or only one (either one), and manufacturers cannot obtain more than their contribution to the profit $\Pi_{1}^{*}$ generated by $R_{1}$; they may however obtain less than their contribution, as manufacturers must match each other's generosity. By contrast, as $M_{B}$ is now the sole supplier of $R_{2}$, it can appropriate all the profit $\Pi_{2}^{*}$ generated by $R_{2}$. Note however that, while $M_{B}$ obtains all of $\Pi_{2}^{*}, M_{A}$ can obtain a larger share of $\Pi_{1}^{*}$ than $M_{B}$, as $\Delta_{A, 1}^{*}>\Delta_{B, 1}^{*}>0 .{ }^{22}$

- Pairwise exclusivity. Suppose now that (pre-existing) pairwise exclusive dealing provisions preclude trade between $M_{A}$ and $R_{2}$ as well as between $M_{B}$ and $R_{1}$. For example, $M_{A}$ and $R_{1}$ as well as $M_{B}$ and $R_{2}$ may have signed exclusive distribution contracts with each other, or $M_{A}$ and $R_{2}$ as well as $M_{B}$ and $R_{1}$ may have signed single branding contracts. We have:

Proposition 3 Suppose that pairwise exclusive dealing provisions preclude trade between, say, $M_{A}$ and $R_{2}$ as well as between $M_{B}$ and $R_{1}$ (i.e., $q_{A 2}^{* *}=q_{B 1}^{* *}=0$ ). Then, there exists a unique equilibrium, characterized as follows:

(i) The quantities $\left(q_{A 1}^{* *}, q_{B 2}^{* *}\right)$ are the same as in the differentiated monoproduct-firm Cournot duopoly: $q_{A 1}^{* *}=q_{B 2}^{* *}=Q^{* *}$.

(ii) Each manufacturer's expected profit is equal to $\alpha \Pi^{* *} / 2$.

Proof. See Appendix D.

The market outcome is thus that of a differentiated duopoly, with one firm offering good $A$ and the other offering good $B$. This equilibrium outcome can be supported by cost-based

${ }^{22}$ That $M_{B}$ 's contribution is positive stems from $q_{B 1}^{*}>0$, which implies

$$
\begin{aligned}
\Pi_{1}^{*} & =\max _{q_{A 1}, q_{B 1}}\left\{\left[P\left(q_{A 1}, q_{B 1}+q_{B 2}^{*}\right)-c\right] q_{A 1}+\left[P\left(q_{B 1}+q_{B 2}^{*}, q_{A 1}\right)-c\right] q_{B 1}\right\} \\
& >\max _{q_{A 1}}\left\{\left[P\left(q_{A 1}, q_{B 2}^{*}\right)-c\right] q_{A 1}\right\} .
\end{aligned}
$$

That $M_{A}$ 's contribution is larger than $M_{B}$ 's follows from the fact that, from (A.1), $P\left(q, q_{B 2}^{*}\right)>P\left(q+q_{B 2}^{*}, 0\right)$ for any $q$ such that $P\left(q, q_{B 2}^{*}\right)>0$. 
two-part tariffs. However, in contrast to the previous cases, it could be supported as well by manufacturers each offering a single forcing contract. Furthermore, equilibrium profits are here unique as well, as proposers always appropriate all the profits generated by their channels.

\subsection{Adoption of Exclusive Dealing Provisions}

To analyze firms' incentives to engage in exclusive dealing, we first discuss the impact of exclusive dealing on firms' profits, and then consider a simple game where firms decide whether to sign exclusivity provisions. We will henceforth assume the following:

(P.2) Comparing the three Cournot duopoly settings introduced in Section 3, total industry profit is higher when a good is produced by a single firm than when it is produced by both firms: $\Pi^{* *}>\Pi^{*}>\Pi^{\circ}$. In addition: (i) output levels are higher when both firms carry both goods than when they both carry a single good: $Q^{* *}<Q^{\circ}$; and (ii) a monoproduct firm sells less when its rival produces both goods (asymmetric duopoly) than when it produces a single good (differentiated monoproduct-firm duopoly).

Property (P.2) asserts that intrabrand competition tends to dissipate profits; as a result, compared with the differentiated monoproduct-firm duopoly setting where each good is produced by a single firm, total industry profit is lower when one good is sold by both firms (asymmetric duopoly), and even more so when both firms produce both goods (multiproduct-firm duopoly). In the Online Appendix B we provide general conditions ensuring that Property (P.2) holds.

This property allows us to provide the following comparative statics:

\section{Proposition 4 We have:}

(i) In any equilibrium that arises in the absence of exclusivity, at least one manufacturerretailer pair $M_{i}-R_{h}$ obtains a lower joint profit than in any equilibrium that arises when $M_{i}$ deals exclusively with $R_{h}$, regardless of whether the rival manufacturer $M_{j}$ deals with both retailers (i.e., under single exclusivity), or exclusively with the rival retailer $R_{k}$ (i.e., under pairwise exclusivity).

(ii) In any equilibrium that arises under single exclusivity, the joint profit of the manufacturerretailer pair that engages in exclusive distribution is higher, and the joint profit of the other pair is lower, than in the unique equilibrium that arises under pairwise exclusivity. 
Proof. See Appendix E.

These profit comparisons build on two intuitive insights. First, industry profits are larger under pairwise exclusivity than under any other configuration; this derives from the fact that, under pairwise exclusivity, each good is sold only by a single firm; by contrast, in all other situations at least one good is sold by more than one firm, which further intensifies competition by creating intrabrand as well as interbrand competition.

Second, a manufacturer-retailer pair obtains a larger joint profit when it is the only pair that engages in exclusive distribution, than under pairwise exclusive dealing. This follows from the fact that, under single exclusivity, the manufacturer-retailer pair that has signed an exclusive distribution agreement must at least obtain what it could jointly achieve if the retailer were to deviate and drop the rival brand, leading to a situation where each good is sold by a single firm, but in which the deviating retailer would moreover face a less aggressive rival than in the pairwise exclusive dealing equilibrium. ${ }^{23}$

Proposition 4 implies that, starting from a situation without any exclusivity, at least one manufacturer-retailer pair would benefit from signing an exclusive distribution contract (whether or not the other pair does the same). Furthermore, if one pair signs an exclusive distribution agreement, then the other pair benefits from doing the same, at the expense of the first pair.

To analyze further firms' incentives to adopt exclusivity provisions, consider the following adoption game: ${ }^{24}$

- Stage 1: One manufacturer, say $M_{i}$, and one retailer, say $R_{h}$, get the opportunity to negotiate an exclusive deal:

- With probability $\beta, M_{i}$ can propose exclusive dealing provisions, namely exclusive distribution and/or single branding agreements, together with a lump-sum transfer; $R_{h}$ can then accept or reject the offer.

- With probability $1-\beta, R_{h}$ can make such an offer, which $M_{i}$ can then accept or reject.

- Stage 2: The two rivals, $M_{j}$ and $R_{k}$ (with $j \neq i \in\{A, B\}$ and $k \neq h \in\{1,2\}$ ), get a similar opportunity.

\footnotetext{
${ }^{23}$ If, for instance, $M_{A}$ and $R_{1}$ sign an exclusive distribution agreement, then $R_{2}$ sells more of good $B$ under pairwise exclusivity, where it expects to be the sole distributor of that good, than under single exclusivity, where $R_{1}$, too, sells good $B$; hence, $q_{B 2}^{*}<q_{B 2}^{* *}$.

${ }^{24}$ The payoffs of the game are those in the induced equilibrium of the model in Section 2.
} 
The following proposition confirms the previous insights:

Proposition 5 The above adoption game has a subgame perfect equilibrium, and all subgame perfect equilibria yield pairwise exclusivity.

\section{Proof. See Appendix F.}

What is remarkable about Proposition 5 is that its conclusion depends neither on firms' bargaining power (as captured by the parameters $\alpha$ and $\beta$ ), nor on equilibrium selection (that is, on how profits are shared in the absence of exclusivity, or under single exclusivity).

The intuition is as follows. As noted above, industry profit is maximal under pairwise exclusivity, which removes any intrabrand competition. Furthermore, from Proposition 4, under single exclusive dealing, the manufacturer-retailer pair that has signed an exclusive distribution agreement obtains a larger joint profit than the other pair. Therefore, in any equilibrium, either the first pair adopts both exclusive distribution and single branding provisions, or each pair successively signs an exclusive distribution agreement.

To conclude this discussion, we first note that the above insights carry over as long as exclusive distribution agreements are available, regardless of whether single branding provisions are also available. In equilibrium, the first pair then signs an exclusive distribution agreement, which induces the other pair to do the same. ${ }^{25}$

By contrast, the incentives to opt for exclusivity are less clear when only single branding provisions are available. Indeed, if one manufacturer-retailer pair, say $M_{j}-R_{k}$, has already opted for single branding, then the other pair, $M_{i}-R_{h}$, will not follow suit: As we have seen, $M_{i}$ and $R_{h}$ 's joint profit is larger in the single exclusive dealing situation where $M_{i}$ does not deal with $R_{k}$, than in case of pairwise exclusivity. Consider now a situation without exclusivity, and suppose that $M_{j}$ and $R_{k}$ sign a single branding contract that prevents $R_{k}$ from dealing with the rival manufacturer $M_{i}$. Intuitively, this eliminates intrabrand competition for good $A$, and may thereby increase total industry profit. However, the above analysis reveals that the other manufacturer-retailer pair, say $M_{i}-R_{h}$, gets the bigger share of that profit; hence, even if total industry profit is increased, $M_{j}$ and $R_{h}$ may obtain too small a share of that bigger pie, making single branding unprofitable. Indeed, in the linear demand example considered at the end of Section 5.3, starting from a situation without exclusivity where the manufacturer-retailer pairs $M_{i}-R_{h}$ and $M_{j}-R_{k}$ share the industry profit equally, then none of them can increase its joint

\footnotetext{
${ }^{25}$ An alternative equilibrium, inducing the same outcome, has the first pair entering into both exclusive distribution and single-branding, thereby unilaterally imposing pairwise exclusivity.
} 
profit by opting for single branding; in the same vein, $M_{j}$ and $R_{k}$ cannot benefit from signing a single branding contract if this does not allow $M_{j}$ to extract more profit from the other retailer, $R_{h}$ (i.e., if $\pi_{j, h}^{*} \leq \pi_{j, h}^{\circ}$ ).

Finally, we note that exclusivity would likely to be less attractive if retailers were differentiated. In that case, the adoption of exclusive dealing provisions would involve a trade-off: on the one hand, they would eliminate intrabrand competition, enabling firms to maintain higher prices; on the other hand, they would limit the volume of sales, for any given prices. The more differentiated the retailers would be, the more important the latter effect would be, making exclusive dealing less appealing. ${ }^{26}$

\subsection{Welfare Effects of Exclusive Dealing}

We now turn to the welfare effects of exclusive dealing. For given quantities $Q_{A}$ and $Q_{B}$, consumer surplus can be expressed as

$$
S\left(Q_{A}, Q_{B}\right) \equiv U\left(Q_{A}, Q_{B}\right)-P\left(Q_{A}, Q_{B}\right) Q_{A}-P\left(Q_{B}, Q_{A}\right) Q_{B}
$$

where

$$
U\left(Q_{A}, Q_{B}\right) \equiv \int_{0}^{Q_{A}} P\left(q_{A}, Q_{B}\right) d q_{A}+\int_{0}^{Q_{B}} P\left(q_{B}, 0\right) d q_{B}
$$

and social welfare can be expressed as

$$
W\left(Q_{A}, Q_{B}\right) \equiv \int_{0}^{Q_{A}}\left[P\left(q_{A}, Q_{B}\right)-c\right] d q_{A}+\int_{0}^{Q_{B}}\left[P\left(q_{B}, 0\right)-c\right] d q_{B} .
$$

We first show that pairwise exclusive dealing increases profits at the expense of consumer surplus and social welfare:

Proposition 6 Consumer surplus and social welfare are lower under pairwise exclusivity than in the absence of any exclusive dealing: $S\left(Q^{* *}, Q^{* *}\right)<S\left(Q^{\circ}, Q^{\circ}\right)$ and $W\left(Q^{* *}, Q^{* *}\right)<W\left(Q^{\circ}, Q^{\circ}\right)$.

\section{Proof. See Appendix G.}

The intuition is straightforward. Exclusive dealing reduces the number of retailers selling any given good, leading to less intense competition, lower outputs and higher prices. This increases

\footnotetext{
${ }^{26}$ We would expect exclusive dealing to remain attractive when retailers are close substitutes. By contrast, in the polar case where retailers are fully differentiated (i.e., local monopolies), industry profit is maximal when all channels are active (and retailers act as "common agents" for the manufacturers), and there is clearly no scope for exclusivity.
} 
firms' profits (as output levels remain above monopoly levels), but obviously harms consumers and reduces total welfare (as prices move further away from marginal cost).

We now show that, under reasonable assumptions, similar insights apply when a single exclusive dealing provision prevents one retailer from distributing one of the goods. To introduce these assumptions, consider a hypothetical duopoly setting, in which firm 1, say, produces both goods at $\operatorname{cost} c$, whereas the other firm produces one good - good $A$, say - at cost $\hat{c} \geq c$, and the other good at $\operatorname{cost} c$. For firm 1, maximizing its profit, i.e.,

$$
\max _{q_{A 1}, q_{B 1}}\left[P\left(Q_{A}, Q_{B}\right)-c\right] q_{A 1}+\left[P\left(Q_{B}, Q_{A}\right)-c\right] q_{B 1}
$$

leads to the following first-order conditions:

$$
\begin{aligned}
& P\left(Q_{A}, Q_{B}\right)-c+\partial_{1} P\left(Q_{A}, Q_{B}\right) q_{A 1}+\partial_{2} P\left(Q_{B}, Q_{A}\right) q_{B 1}=0 \\
& P\left(Q_{B}, Q_{A}\right)-c+\partial_{1} P\left(Q_{B}, Q_{A}\right) q_{B 1}+\partial_{2} P\left(Q_{A}, Q_{B}\right) q_{A 1}=0 .
\end{aligned}
$$

Likewise, for firm 2,

$$
\max _{q_{A 2}, q_{B 2}}\left[P\left(Q_{A}, Q_{B}\right)-\hat{c}\right] q_{A 2}+\left[P\left(Q_{B}, Q_{A}\right)-c\right] q_{B 2}
$$

leads to:

$$
\begin{aligned}
& P\left(Q_{A}, Q_{B}\right)-\hat{c}+\partial_{1} P\left(Q_{A}, Q_{B}\right) q_{A 2}+\partial_{2} P\left(Q_{B}, Q_{A}\right) q_{B 2}=0 \\
& P\left(Q_{B}, Q_{A}\right)-c+\partial_{1} P\left(Q_{B}, Q_{A}\right) q_{B 2}+\partial_{2} P\left(Q_{A}, Q_{B}\right) q_{A 2}=0 .
\end{aligned}
$$

Adding-up (1) and (3), and doing the same with (2) and (4), yields:

$$
\begin{array}{r}
2 P\left(Q_{A}, Q_{B}\right)-c-\hat{c}+\partial_{1} P\left(Q_{A}, Q_{B}\right) Q_{A}+\partial_{2} P\left(Q_{B}, Q_{A}\right) Q_{B}=0 \\
2 P\left(Q_{B}, Q_{A}\right)-2 c+\partial_{1} P\left(Q_{B}, Q_{A}\right) Q_{B}+\partial_{2} P\left(Q_{A}, Q_{B}\right) Q_{A}=0 .
\end{array}
$$

The first condition can be interpreted as characterizing the "aggregate best-response" $Q_{A}=$ $\hat{R}\left(Q_{B}, \hat{c}\right)$, which determines how much of good $A$ is produced, for a given aggregate quantity of good $B$; likewise, the second condition characterizes the aggregate best-response $Q_{B}=R\left(Q_{A}\right)$. We will assume that this hypothetical duopoly game has a well-behaved equilibrium, namely:

(B) For any $Q>0,-1<R^{\prime}(Q) \leq 0$, with a strict inequality when $R(Q)>0,-1<$ $\partial_{1} \hat{R}(Q, \hat{c}) \leq 0$, and $\partial_{2} \hat{R}(Q, \hat{c}) \leq 0$, with strict inequalities when $\hat{R}(Q, \hat{c})>0$.

The first part of this assumption amounts to asserting that aggregate quantities are strategic substitutes, and that the resulting equilibrium is unique and "stable;" ${ }^{27}$ the second part simply

\footnotetext{
${ }^{27}$ That is, a standard tâtonnement process would converge towards the equilibrium.
} 
asserts that an increase in the cost of a good leads to a reduction in the aggregate production of that good. These properties are satisfied for instance when demand is linear; in the Online Appendix $\mathrm{C}$, we provide more general conditions on demand ensuring that Assumption $(B)$ holds. We have:

Proposition 7 Consumer surplus and social welfare are lower under single exclusivity than in the absence of any exclusive dealing.

\section{Proof. See Appendix H.}

Intuitively, we would moreover expect the impact of exclusivity to be more important when goods $A$ and $B$ are more differentiated. Indeed, exclusive dealing has no effect in the limit case of perfect substitutes (as, in that case, the retailers do not care about whether they sell one good or both of them), and enables instead firms to achieve the industry-wide monopoly outcome when goods $A$ and $B$ face independent demands. To illustrate this, consider the case of linear demand:

$$
P\left(Q_{A}, Q_{B}\right)=1-\frac{Q_{A}+s Q_{B}}{1+s},
$$

where $s$ reflects the degree of substitution between $A$ and $B$, and ranges from $s=0$ (independent demands) to $s=1$ (perfect substitutes). Normalizing the production cost to $c \equiv 0$, we have:

$$
\begin{aligned}
Q^{* *} & =q^{* *}=\frac{1+s}{2+s} \leq Q^{\circ}=2 q^{\circ}=\frac{2}{3} \\
p^{* *} & =P\left(Q^{* *}, Q^{* *}\right)=\frac{1}{2+s} \leq p^{\circ}=P\left(Q^{\circ}, Q^{\circ}\right)=\frac{1}{3},
\end{aligned}
$$

with strict inequalities whenever $s<1$. Hence, not only does pairwise exclusivity reduce output and raise prices, but the impact is greater when the goods $A$ and $B$ are more differentiated: The demand is here normalized so as to be independent of the differentiation parameter $s$ in symmetric configurations, ${ }^{28}$ and neither $Q^{\circ}$ nor $p^{\circ}$ depend on $s$ either; yet, $Q^{* *}$ decreases, and $p^{* *}$ increases, as $s$ decreases.

Intuitively, we would also expect each exclusive dealing provision to contribute to increasing profit, at the expense of consumers and allocative efficiency. This is indeed the case in the above linear model. When a single exclusive dealing provision precludes trade between $M_{A}$ and $R_{2}$,

\footnotetext{
${ }^{28}$ That is, $P(Q, Q)=1-Q$ and thus remains constant (for symmetric configurations) when the degree of product differentiation varies. In particular, the benchmark monopoly quantity, $Q^{M}=1 / 2$, is independent of $s$.
} 
the equilibrium prices and quantities are uniquely defined and given by:

$$
\begin{aligned}
q_{A 1}^{*} & =\frac{1}{2}<Q^{\circ}=\frac{2}{3}, q_{B 1}^{*}=\frac{2-s}{6}<q^{\circ}=\frac{1}{3}<q_{B 2}^{*}=\frac{1+s}{3}, \\
Q_{A}^{*} & =\frac{1}{2}<Q^{\circ}=\frac{2}{3}<Q_{B}^{*}=\frac{4+s}{6}, \\
p^{* *} & =\frac{1}{2+s}>p_{A}^{*}=\frac{1}{2}-\frac{s}{6}>p_{B}^{*}=p^{\circ}=\frac{1}{3} .
\end{aligned}
$$

That is, starting from the baseline scenario with no exclusivity, shutting down the channel $M_{A}-R_{2}$ induces $R_{1}$ to sell more of good $A$, but not so much as to compensate for $R_{2}$ 's lost sales of good $A$; this also induces $R_{2}$ to sell more of good $B$, a move partially offset by $R_{1}$ reducing its own sales of that good (both because it faces a more aggressive rival $R_{2}$ for good $B$, and because $R_{1}$ itself sells more of the substitute good $A$ ). As one price increases, the other one remaining constant, consumer surplus and social welfare decrease, whereas industry profit increases.

It can be checked that each exclusivity provision increases industry profit, and reduces both consumer surplus and social welfare:

$$
\begin{aligned}
\Pi^{* *} & =2 p^{* *} Q^{* *}=2 \frac{1+s}{(2+s)^{2}}>\Pi^{*}=p_{A}^{*} Q_{A}^{*}+p_{B}^{*} Q_{B}^{*}=\frac{17-s}{36}>\Pi^{\circ}=2 p^{\circ} Q^{\circ}=\frac{4}{9}, \\
S^{* *} & =S\left(Q^{* *}, Q^{* *}\right)=\frac{(1+s)^{2}}{(2+s)^{2}}<S^{*}=S\left(Q_{A}^{*}, Q_{B}^{*}\right)=\frac{25+7 s}{72}<S^{\circ}=S\left(Q^{\circ}, Q^{\circ}\right)=\frac{4}{9}, \\
W^{* *} & =S^{* *}+\Pi^{* *}=\frac{3+4 s+s^{2}}{(s+2)^{2}}<W^{*}=\Pi^{*}+S^{*}=\frac{59+5 s}{72}<W^{\circ}=S^{\circ}+\Pi^{\circ}=\frac{8}{9} .
\end{aligned}
$$

This is illustrated by Figure 1, which shows furthermore that the impact of each exclusivity provision is also larger when the goods are more differentiated: The top curves depict the industry profit and the bottom ones depict consumer surplus, as a function of the substitution parameter $s$; the bold line represents the non-exclusivity benchmark, whereas the dashed curves 
correspond to single exclusivity, and the solid curves to pairwise exclusivity.

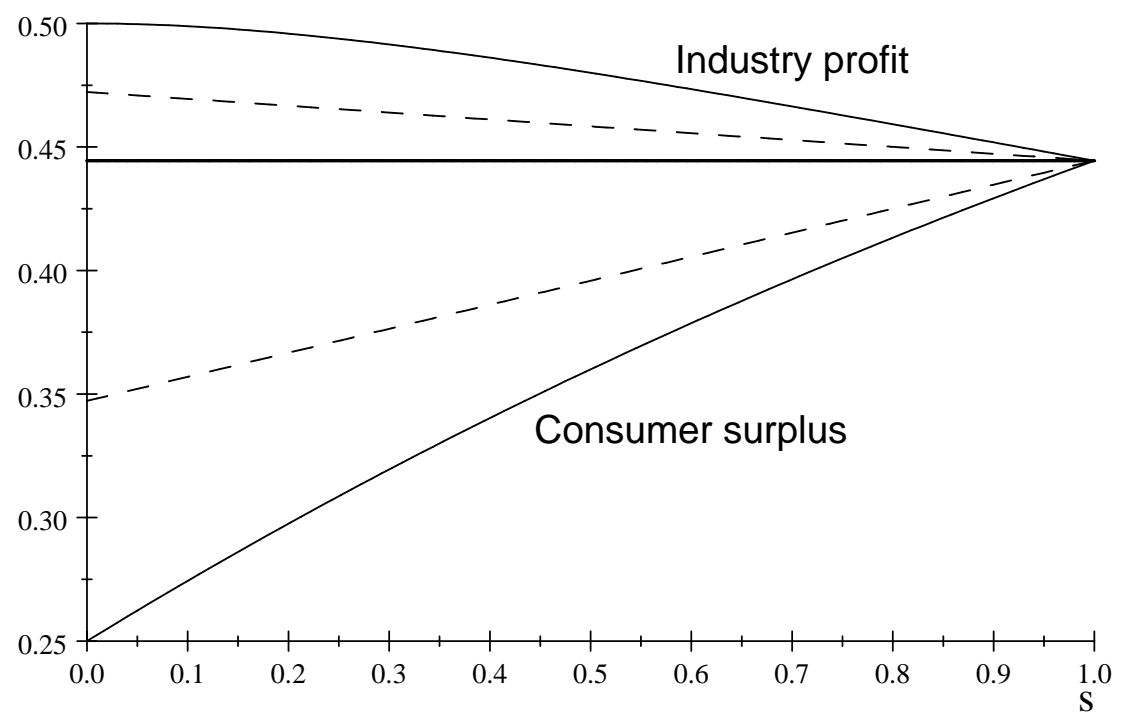

Figure 1: Profit and consumer surplus for a linear demand.

\section{Vertical Integration}

In this section, we analyze the positive and normative effects of vertical integration. We begin by considering the case of two vertically integrated firms (pairwise vertical integration), and then turn to the case where one vertically integrated firm faces independent rivals (single vertical integration). We show that, under mild regularity conditions, pairwise vertical integration yields a unique equilibrium, in which each vertically integrated firm forecloses its rival; the equilibrium outcome thus replicates (in terms of retail prices and quantities) that of pairwise exclusivity. Under single vertical integration, there exists an equilibrium in which the integrated firm forecloses its downstream rival, thus replicating the outcome of single exclusive dealing. That is, a vertical merger leads to the foreclosure of the rival retailer. The welfare analysis of vertical integration therefore mirrors that of exclusive dealing: vertical integration reduces both consumer surplus and social welfare.

To state our regularity conditions, we first make an excursion and introduce a related duopoly game. 


\subsection{Excursion: Related Duopoly Game}

Consider the following hypothetical duopoly game, denoted $\Gamma$. Two firms, 1 and 2, offer two goods, $A$ and $B$. Firm 1 chooses its quantity $q_{A 1}$ of good $A$ while firm 2 chooses its quantity $q_{B 2}$ of good $B$. In addition, firm 1 sells an exogenous quantity $\hat{q}_{B 1}$ of good $B$ and firm 2 an exogenous quantity $\hat{q}_{A 2}$ of good $A$, so that the profit functions of the two firms are given by

$$
\hat{\Pi}_{1}\left(q_{A 1}, q_{B 2} ; \hat{q}_{B 1}, \hat{q}_{A 2}\right) \equiv \Pi_{1}\left(q_{A 1}, \hat{q}_{B 1} ; \hat{q}_{A 2}, q_{B 2}\right)
$$

and

$$
\hat{\Pi}_{2}\left(q_{A 1}, q_{B 2} ; \hat{q}_{B 1}, \hat{q}_{A 2}\right) \equiv \Pi_{2}\left(\hat{q}_{A 2}, q_{B 2} ; q_{A 1}, \hat{q}_{B 1}\right) .
$$

In the special case where $\hat{q}_{A 2}=\hat{q}_{B 1}=0$, this game simplifies to the differentiated monoproductfirm Cournot duopoly introduced in Section 3, where each good is sold by only one firm.

We will assume that this game $\Gamma$ has the following properties:

(Г.1) Game $\Gamma$ has a unique Nash equilibrium, $\left(\tilde{q}_{A 1}\left(\hat{q}_{A 2}, \hat{q}_{B 1}\right), \tilde{q}_{B 2}\left(\hat{q}_{B 1}, \hat{q}_{A 2}\right)\right)$.

We will denote firm $i$ 's equilibrium profit by $\tilde{\Pi}_{i}\left(\hat{q}_{B 1}, \hat{q}_{A 2}\right)$.

(Г.2) The aggregate equilibrium profit,

$$
\tilde{\Pi}\left(\hat{q}_{B 1}, \hat{q}_{A 2}\right)=\tilde{\Pi}_{1}\left(\hat{q}_{B 1}, \hat{q}_{A 2}\right)+\tilde{\Pi}_{2}\left(\hat{q}_{B 1}, \hat{q}_{A 2}\right)
$$

is uniquely maximized for $\hat{q}_{B 1}=\hat{q}_{A 2}=0$; that is, $\tilde{\Pi}(0,0)>\tilde{\Pi}\left(\hat{q}_{B 1}, \hat{q}_{A 2}\right)$ whenever $\hat{q}_{B 1}+$ $\hat{q}_{A 2}>0$.

(Г.3) The equilibrium quantity $\tilde{q}_{A 1}\left(\hat{q}_{A 2}, 0\right)$ (resp., $\left.\tilde{q}_{B 2}\left(\hat{q}_{B 1}, 0\right)\right)$ is (weakly) decreasing in $\hat{q}_{A 2}$ (resp., $\left.\hat{q}_{B 1}\right)$.

These properties are satisfied in the case of linear demand. In the Online Appendix D, we provide more general sufficient conditions on demand that ensure that $(\boldsymbol{\Gamma} . \mathbf{1})-(\boldsymbol{\Gamma} . \mathbf{3})$ do indeed hold.

\subsection{Pairwise Vertical Integration}

Consider first the case where two firms, say $M_{A}-R_{1}$ and $M_{B}-R_{2}$, are vertically integrated. Intuitively, each manufacturer then has an incentive to protect its own retailer from intrabrand competition. The following proposition shows that pairwise vertical integration leads indeed to complete foreclosure of downstream rivals, mirroring the outcome under pairwise exclusive dealing: 
Proposition 8 Suppose $M_{A}-R_{1}$ and $M_{B}-R_{2}$ are vertically integrated. Then, there exists a unique equilibrium, in which each integrated manufacturer forecloses the rival retailer. The equilibrium thus replicates the outcome of pairwise exclusivity.

Proof. See Appendix I.

The proposition shows that pairwise vertical integration leads to a strong form of foreclosure, as each integrated firm refuses to deal with the other integrated firm. Moreover, combined with Lemma 2, it shows that pairwise vertical integration is "less competitive" than single vertical integration. It follows from our previous welfare analysis that vertical integration harms consumers and society. In particular, under pairwise vertical integration both prices are higher (and consumer surplus as well as social welfare are thus lower) than under vertical separation.

\subsection{Single Vertical Integration}

We now turn to the case where a single upstream-downstream pair, $M_{A}-R_{1}$ say, is vertically integrated. Our previous analysis allows us to provide a very partial characterization of equilibrium:

Lemma 2 Suppose that $M_{A}$ and $R_{1}$ are vertically integrated whereas $M_{B}$ and $R_{2}$ are not. Then, in equilibrium, the unintegrated manufacturer $M_{B}$ signs a cost-based contract with each retailer. The vector of equilibrium quantities, $\left(q_{A 1}^{*}, q_{A 2}^{*}, q_{B 1}^{*}, q_{B 2}^{*}\right)$, is thus such that

$$
q_{B h}^{*}=\arg \max _{q_{B h}}\left[P\left(q_{B h}+q_{B k}^{*}, q_{A h}^{*}+q_{A k}^{*}\right)-c\right] q_{B h}+P\left(q_{A h}^{*}+q_{A k}^{*}, q_{B h}+q_{B k}^{*}\right) q_{A h}^{*}
$$

for all $h \neq k \in\{1,2\}$.

Proof. This is an immediate implication of Lemma 1.

Intuitively, the integrated $M_{A}$ does not need access to $R_{2}$ to sell its good (any unit that $M_{A}$ sells through $R_{2}$ could instead be sold directly through the downstream affiliate $R_{1}$ ), and it has moreover an incentive to protect its own retailer $R_{1}$ from intrabrand competition. To capture this intuition, we will assume that game $\Gamma$ has the following property:

(Г.4) The expressions

$$
\tilde{\Pi}\left(\hat{q}_{A 2}, q_{B 1}^{*}\right)-\max _{q_{B 2}}\left[P\left(q_{B 1}^{*}+q_{B 2}, \tilde{q}_{A 1}\left(\hat{q}_{A 2}, q_{B 1}^{*}\right)\right)-c\right] q_{B 2}
$$

and

$\tilde{\Pi}\left(\hat{q}_{A 2}, q_{B 1}^{*}\right)-\max _{q_{A 1}}\left\{\left[P\left(q_{A 1}, q_{B 1}^{*}+\tilde{q}_{B 2}\left(q_{B 1}^{*}, \hat{q}_{A 2}\right)\right)-c\right] q_{A 1}+\left[P\left(q_{B 1}^{*}+\tilde{q}_{B 2}\left(q_{B 1}^{*}, \hat{q}_{A 2}\right), q_{A 1}\right)-c\right] q_{B 1}^{*}\right\}$ are maximized at $\hat{q}_{A 2}=0$. 
This property holds, for instance, when demand is linear. The following proposition shows that there then exists an equilibrium in which the integrated firm will not supply its downstream rival:

Proposition 9 Suppose that $M_{A}$ and $R_{1}$ are vertically integrated whereas $M_{B}$ and $R_{2}$ are not. Then, there exists an equilibrium in which the integrated $M_{A}$ forecloses the independent $R_{2}$. This equilibrium thus yields the same retail prices and quantities as under single exclusivity, but gives $R_{2}$ a higher expected profit.

\section{Proof. See Appendix J.}

In the equilibrium characterized by the proposition, the vertical merger between $M_{A}$ and $R_{1}$ has the same impact on consumers as an exclusive distribution contract between $M_{A}$ and $R_{1}$, or a single branding contract between $M_{B}$ and $R_{2}$. However, in contrast to the case of exclusive dealing, thanks to the competition between the two manufacturers for its business, the independent $R_{2}$ extracts some rents even when manufacturers are the proposers. In equilibrium, $M_{A}$ makes an attractive offer to $R_{2}$, the anticipation of which prompts $M_{B}$ to make a generous offer to $R_{2}$, which in turn prevents $M_{A}$ from winning the competition for $R_{2}$ 's business.

Remark: On equilibrium uniqueness. When retailers are the proposers, it is shown in Appendix $\mathrm{J}$ that foreclosure of the downstream rival is the unique equilibrium outcome under a mild regularity condition.

Our analysis of exclusive dealing implies that firms have an incentive to integrate vertically: If no firm is vertically integrated, there exists a manufacturer-retailer pair, say $M_{A}$ and $R_{1}$, that can increase its joint profit by merging. Moreover, if $M_{A}$ and $R_{1}$ are vertically integrated, then $M_{B}$ and $R_{2}$ can also increase their joint profit by merging.

We conclude this section by noting that "complete foreclosure" arises here from the fact that a single retailer suffices to serve the entire market. If it were not the case, e.g., due to downstream capacity constraints or to differentiation among the retailers, then integrated manufacturers would still wish to deal with downstream rivals in order to expand market coverage or serve customer niches; in such situations, we would thus expect vertical integration to result into partial rather then complete foreclosure. By the same token, in such situations vertical integration (and partial foreclosure) is likely to be more profitable than exclusive dealing (and thus complete foreclosure). ${ }^{29}$

\footnotetext{
${ }^{29}$ See Rey and Tirole (2007) for an analysis of the impact of downstream differentiation on the extent of foreclosure in the case of an upstream monopoly.
} 


\section{$7 \quad$ Related Literature}

\subsection{Common Agency versus Interlocking Relationships}

A substantial body of literature has studied "common agency" situations in which competing firms deal with the same unique partner. When multiple suppliers deal with a common downstream firm, there is a unique equilibrium in terms of downstream prices and quantities, although the resulting profit can be shared in multiple ways. Furthermore, equilibrium contracts are not only bilaterally efficient, but they also maximize total industry profit. ${ }^{30}$ As a result, there is no incentive to engage in exclusive dealing (that is, single branding) or to integrate vertically. By contrast, when multiple downstream competitors (secretly) negotiate with a common supplier, there is a unique equilibrium outcome (also in terms of profit sharing) and contracts are still bilaterally efficient, but they no longer maximize total industry profit; as a result, firms have an incentive to opt for exclusivity (that is, to sign an exclusive distribution agreement) or vertical integration. ${ }^{31}$

Allowing for "interlocking relationships" in a successive duopoly setting, we show the existence of a unique equilibrium outcome in terms of downstream prices and quantities, and that the resulting profit can be shared in different ways, depending on the type of contracts that are signed. We also find that contracts are not only bilaterally efficient, but also "trilaterally efficient" in that they maximize the joint profit of the retailer with both manufacturers (given the quantities sold by the other retailer); however, they fail to maximize total industry profit. As a result, and regardless of firms' relative bargaining power as well as which equilibrium is selected, firms have an incentive to adopt exclusivity provisions or to integrate vertically. The analysis moreover explores further the distinction between exclusive distribution and single branding provisions. While firms always have an incentive to adopt exclusive distribution agreements, single branding provisions appear to be less attractive.

\subsection{Multilateral Bargaining in Vertically Related Industries}

Modelling multiple interlocking relationships is challenging. Two approaches have been adopted in the literature. The "Nash-in-Nash" approach combines the cooperative "Nash bargaining" solution with the spirit of the non-cooperative "Nash equilibrium": for each vertical pair, the

\footnotetext{
${ }^{30}$ See Bernheim and Whinston $(1985,1986 \mathrm{~b}, 1998)$ and O'Brien and Shaffer (1997). Note that these insights obtain regardless of whether contracts are public or private.

${ }^{31}$ See Hart and Tirole (1990), O’Brien and Shaffer (1992) and McAfee and Schwartz (1994).
} 
equilibrium contract maximizes the joint profit of the two partners (and divides it according to a given sharing rule), ${ }^{32}$ taking as given all other equilibrium contracts. ${ }^{33}$ This, however, implicitly assumes that one firm cannot adjust its behavior in one negotiation, when contemplating a move in another negotiation. The other approach, which we have adopted here, considers instead the (subgame-perfect) Nash equilibrium of a non-cooperative game, in which one side makes take-it-or-leave-it offers to the other side. ${ }^{34}$ However, to allow for arbitrary bargaining power between upstream and downstream partners, as in Chemla (2003) we assume that either side (manufacturers or retailers) gets to make the offers with some probability.

The Nash-in-Nash approach typically assumes that each party assigns different agents to negotiate with their different partners, which moreover do not communicate with each other; a firm thus appears "schizophrenic" in the sense that, when making or receiving an unexpected offer in the negotiation with one partner, it cannot adjust its behavior in its bargaining with other partners. Recently, Collard-Wexler et al. (2015) provide a non-cooperative foundation for Nash-in-Nash bargaining over lump-sum payments, based on simultaneous bilateral negotiations with alternating moves à la Stahl-Rubinstein. However, their approach applies only to a limited set of environments with given, positive gains from trade (that is, the focus is on profit sharing, taking as a given the profit generated by each market structure) and declining returns from additional agreements. It therefore does not apply in the more general setting considered here, where the gains from trade depend also on the contracts signed by the negotiating parties (with each other, and with their other partners), as well as on the contracts signed by the other firms.

\subsection{Economic Environments}

The IO literature on vertically related markets has mostly focused on upstream (or downstream) monopolies, on "competing vertical structures" where each upstream firm deals with a distinct

\footnotetext{
${ }^{32}$ The bilateral Nash bargaining solution indeed entails joint profit maximization when the partners can share this profit anyway they want. This is for instance the case when non-linear tariffs are feasible, as in our setting, or when wholesale prices are negotiated at the same time as, or after, retail pricing decisions, as often assumed in the empirical literature on healthcare or cable-TV networks.

${ }^{33}$ This approach was pioneered by Crémer and Riordan (1987) and Horn and Wolinsky (1988). Recent empirical and theoretical work includes Crawford and Yurukoglu (2012), Crawford et al. (2015), Ho and Lee (2015), de Fontenay and Gans (2014) and Collard-Wexler et al. (2015).

${ }^{34}$ Seminal contributions applying this approach to interlocking vertical relations include Ordover, Saloner and Salop (1990), Hart and Tirole (1990) and McAfee and Schwartz (1994).
} 
set of downstream firms (e.g., franchise networks), ${ }^{35}$ or on homogeneous suppliers. ${ }^{36}$ The other papers differ from ours in terms of the types of contracts that are considered or their observability.

\subsubsection{Secret versus Public Contracting}

Much of the existing literature on vertically related markets assumes that wholesale tariffs are publicly observed by all firms, which gives rise to pre-commitment effects, with firms departing from bilateral efficiency for purely strategic reasons. ${ }^{37}$ However, this assumption is not very plausible in many industries, where the terms of supply contracts are kept highly confidential. Furthermore, as noted by Hart and Tirole (1990), while firms may make their contracts public so as to influence their rivals' behavior, they have an incentive to renegotiate the terms privately so as to achieve bilateral efficiency.

Throughout the paper, we thus focus on secret contracting, and assume that the terms of an agreement as well as acceptance decisions are private information to the two contracting parties, and not observed by rival firms. ${ }^{38}$

\subsubsection{On the Class of Contracts}

The papers studying interlocking vertical relationships often restrict attention to particular types of (public) contracts such as linear tariffs or two-part tariffs. For instance, Lee and Fong (2013) and Collard-Wexler et al. (2015) focus on the division of gains from trade and restrict attention to lump-sum payments, treating the reduced-form profits from a given network structure as primitives. Other papers are relying on linear tariffs, ${ }^{39}$ which are bilaterally inefficient as they create double-marginalization problems. As a result, vertical integration involves efficiency gains

\footnotetext{
${ }^{35}$ Papers featuring competing vertical structures include Bonanno and Vickers (1988), Rey and Stiglitz (1988, 1995), Gal-Or (1991), Jullien and Rey (2007), and Piccolo and Miklós-Thal (2012).

${ }^{36}$ See, e.g., Nocke and White $(2007,2010)$.

${ }^{37}$ See footnote 1 for references.

${ }^{38}$ Seminal papers on secret contracting in vertically related markets include Hart and Tirole (1990), O'Brien and Shaffer (1992) and McAfee and Schwartz (1994).

${ }^{39}$ See, e.g., Dobson and Waterson (2007) and Crawford and Yurukoglu (2012). In much of the recent empirical literature on the U.S. health care industry, including Gowrisankaran, Nevo, and Town (2015), Ho and Lee (2015), and Crawford et al. (2015), the contracts between upstream and downstream firms (hospitals and insurers, respectively) are assumed to be linear. In these papers, hospital prices are assumed to be determined either after, or simultaneously with, downstream prices, implying that the negotiation of the hospital prices focuses on the division of fixed gains from trade.
} 
(by eliminating double marginalization problems) as well as strategic motives. De Fontenay and Gans $(2005,2014)$ focus instead on quantity forcing contracts. Finally, a few papers have considered two-part tariffs,${ }^{40}$ which are bilaterally efficient, but impose a given division of profits; we show that other contracts can be used to sustain different divisions of the industry profit.

We allow here for general contracts, thereby enabling firms to achieve bilateral efficiency. So doing also allows for a sharper welfare analysis that focuses on the strategic effects of exclusive dealing and vertical integration.

Finally, following most of the vertical contracting literature, we restrict attention to purely bilateral agreements that cannot condition on trade taking place with other partners. ${ }^{41}$ In contrast, de Fontenay and Gans (2014) consider contracts that are explicitly contingent on the set of active channels. They establish the existence of an equilibrium in which profit is distributed according to the Myerson-Shapley value, which thus gives each supplier a sizeable share of the profit, even in the case of perfectly substitutable suppliers. By contrast, in our setting, manufacturers never obtain more than their contribution to the industry profit; their profits thus go to zero as they become closer substitutes, regardless of their bargaining power in the bilateral negotiations.

\section{Conclusion}

In this paper, we develop a tractable framework for the analysis of interlocking bilateral relationships in vertically related markets. Key features of the framework include the absence of any restriction on the contracts that can be signed (e.g., lump-sum payments, linear or two-part tariffs, forcing contracts, and so forth), secret contracting (i.e., contract terms are private information to the contracting parties), balanced bargaining power in each bilateral negotiation, product differentiation in the upstream market and quantity competition in the downstream market.

We first characterize the equilibrium outcomes in the absence of any exclusive dealing provision and vertical integration. We show that all channels are then active, and that they trade at bilaterally efficient levels; as a result, the equilibrium downstream prices and quantities are unique, and they replicate the outcome of a multiproduct-firm Cournot oligopoly. This equilib-

\footnotetext{
${ }^{40}$ See, e.g., Rey and Vergé (2010) and Allain and Chambolle (2011).

${ }^{41}$ Firms can, however, use menus of options (implicitly) designed for different network structures, and indeed must do so in equilibrium (see the discussion of bumping problems in Section 4).
} 
rium outcome cannot be sustained by simple forcing contracts, but can be sustained by two-part tariffs, for instance, or by menus of forcing contracts (with an option designed for exclusivity, and another one designed for non-exclusivity). How the resulting profit is shared among firms depends not only on their bargaining power, however, but also on the type of tariffs that are used. When two-part tariffs or similar contracts are used, manufacturers obtain the full share of their contribution to the profits generated by retailers. By contrast, with other tariffs retailers can obtain a larger share of the profits that they generate - and can even appropriate all profits, regardless of their market power. These tariffs must however offer below-cost prices for units beyond the equilibrium levels.

We use this framework to shed some light on a long-standing debate on vertical foreclosure. More specifically, we analyze the positive and normative effects of exclusive dealing and vertical integration, and show that, from a purely strategic standpoint, firms have an incentive to engage in exclusive dealing or vertical integration to exert more market power, at the expense of consumers and society. Surprisingly, this is the case regardless of firms' relative bargaining power, as well as of which equilibrium arises (and thus, of the division of profits between upstream and downstream firms) before and after the adoption of an exclusive dealing agreement or a vertical merger.

For simplicity, we focus here on the strategic effects of vertical integration and exclusive dealing, and show that they give rise to antitrust concerns. However, as is well-known, these vertical agreements can also generate efficiencies. Vertical integration can, for instance, eliminate double marginalization problems, and more generally these arrangements can be used to align the interests and incentives of the trading partners. Yet, our analysis suggests that there may be a trade-off from an antitrust policy standpoint.

For the sake of exposition, we make a number of simplifying assumptions. The arguments and the underlying intuition however make clear that many of these could easily be relaxed at the cost of heavier notation and a more cumbersome exposition. In particular, the framework could be extended to any number of firms, with arbitrary costs, in the upstream and downstream markets, to asymmetric upstream differentiation, or to different bargaining power across firms or across channels. A more substantial extension would account for product differentiation in the downstream market. As discussed in Sections 5.2 and 6.3, in this case, exclusive dealing provisions are likely to be less attractive, and vertical integration may no longer lead to complete foreclosure of rival retailers.

A more challenging avenue for future research involves analyzing downstream price compe- 
tition, which is known to raise complex issues for the treatment of out-of-equilibrium beliefs. Another exciting avenue for future research consists in studying the positive and normative effects of other contractual arrangements, such as "fidelity rebates" based on market shares, Most-Favored-Nation clauses, or agency contracts. 


\section{Appendix}

\section{A Proof of Lemma 1}

Consider first the case where the manufacturers are the proposers, and fix a candidate equilibrium, with associated equilibrium quantities $\left(q_{i h}^{e}\right)_{i=A, B, h=1,2}$ and acceptance decisions $\left(\delta_{i h}^{e}\right)_{i=A, B, h=1,2}$, with the convention that $\delta_{i h}^{e}=1$ if $M_{i}$ and $R_{h}$ are vertically integrated and, when they are independent, $\delta_{i h}^{e}=1$ if the offer is accepted and $\delta_{i h}^{e}=0$ if it is not (in which case $q_{i h}^{e}=0$ ). Suppose further that, in this equilibrium, the unintegrated $M_{i}$ signs with $R_{h}$ a contract that is not cost-based, i.e., $q_{i h}^{e} \notin \chi\left(q_{i k}^{e}, q_{j h}^{e}, q_{j h}^{e}\right)$. Consider now a deviation by $M_{i}$ to a cost-based two-part tariff $\left(c, \tilde{F}_{i h}\right)$, where the fixed fee $\tilde{F}_{i h}$ is as follows:

- if $R_{h}$ is vertically integrated with $M_{j}$, then:

$$
\begin{aligned}
\tilde{F}_{i h}= & \max _{q_{i h}}\left\{\left[P\left(q_{i h}+q_{i k}^{e}, q_{j h}^{e}+q_{j k}^{e}\right)-c\right] q_{i h}\right. \\
& \left.+\left[P\left(q_{j h}^{e}+q_{j k}^{e}, q_{i h}+q_{i k}^{e}\right)-c\right] q_{j h}^{e}+\delta_{j k}^{e}\left[\tau_{j k}^{e}\left(q_{j k}^{e}\right)-c q_{j k}^{e}\right]\right\}-\pi_{j-h}^{e},
\end{aligned}
$$

where $\pi_{j-h}^{e}$ denotes the profit of the integrated firm $M_{j}-R_{h}$ in the candidate equilibrium. The terms in curly brackets represent the profit (gross of the fixed fee $\tilde{F}_{i h}$ ) that the vertically integrated firm $M_{j}-R_{h}$ would make if $R_{h}$ accepted $M_{i}$ 's deviant offer and maintained the equilibrium quantity $q_{j h}^{e}$, and $R_{k}$ maintained the equilibrium quantities $q_{i k}^{e}$ and $q_{j k}^{e}$ :

- the first two terms are the profits generated by, respectively, the channels $M_{i}-R_{h}$ and $M_{j}-R_{h}$,

- whereas the third term is the profit that $M_{j}$ generates in equilibrium through the sales to the other, unintegrated retailer $R_{k}$.

- if instead $R_{h}$ is not vertically integrated, then:

$$
\begin{aligned}
\tilde{F}_{i h}=\max _{q_{i h}}\left\{\left[P\left(q_{i h}+q_{i k}^{e}, \delta_{j h}^{e} q_{j h}^{e}+q_{j k}^{e}\right)-c\right] q_{i h}\right. \\
\left.+\delta_{j h}^{e}\left[P\left(q_{j h}^{e}+q_{j k}^{e}, q_{i h}+q_{i k}^{e}\right) q_{j h}^{e}-\tau_{j h}^{e}\left(q_{j h}^{e}\right)\right]\right\}-\pi_{h}^{e},
\end{aligned}
$$

where $\pi_{h}^{e}$ denotes the profit that the unintegrated $R_{h}$ makes in equilibrium. The terms in curly brackets represent the profit (gross of the fixed fee $\tilde{F}_{i h}$ ) that the unintegrated $R_{h}$ would make if it accepted $M_{i}$ 's deviant offer and maintained its acceptance decision $\delta_{j h}^{e}$ 
vis-à-vis $M_{j}$ 's contract offer as well as the equilibrium quantity $q_{j h}^{e}$, and $R_{k}$ maintained the equilibrium quantities $q_{i k}^{e}$ and $q_{j k}^{e}$ :

- the first term is the profit generated by the channel $M_{i}-R_{h}$,

- whereas the second term is the profit that $R_{h}$ makes on its contract with $M_{j}$.

We first claim that $R_{h}$ is willing to accept the deviant offer $\left(c, \tilde{F}_{i h}\right)$ :

1. As wary beliefs boil down to passive beliefs in this situation, at the acceptance stage $R_{h}$ continues to believe that its downstream rival $R_{k}$ has been offered the equilibrium contracts and will sell the equilibrium quantities $q_{i k}^{e}$ and $q_{j k}^{e}$ in the continuation game.

2. By accepting $M_{i}$ 's deviant offer, $R_{h}$ can make the same profit as in the candidate equilibrium by sticking to its acceptance decision vis-à-vis $M_{j}$ 's equilibrium offer and maintaining the quantity $q_{j h}$ at its equilibrium level $q_{j h}^{e}$, and can do only better by optimizing over these decisions.

3. If instead $R_{h}$ rejects $M_{i}$ 's deviant offer, it obtains the same profit as in the continuation game following the rejection of $M_{i}$ 's equilibrium offer. By construction, this cannot exceed $M_{i}$ 's equilibrium profit: it constitutes the equilibrium profit if in equilibrium $R_{h}$ rejects $\tau_{i h}^{e}$, and must be (weakly) lower otherwise.

As $R_{h}$ is willing to accept this deviant offer (and can be induced to do so, if needed, by slightly reducing the fixed fee $\tilde{F}_{i h}$ ), which gives $M_{i}$ a profit equal to $\tilde{F}_{i h}$, this deviation is unprofitable only if $\tilde{F}_{i h} \leq \pi_{i, h}^{e}$, where

$$
\pi_{i, h}^{e}=\delta_{i h}^{e}\left[\tau_{i h}^{e}\left(q_{i h}^{e}\right)-c q_{i h}^{e}\right]
$$

denotes the equilibrium profit that $M_{i}$ makes from selling through retailer $R_{h}$. But then:

- If $R_{h}$ is vertically integrated with $M_{j}$ (implying $\delta_{i h}^{e}=1$ ), we can rewrite $\pi_{i, h}^{e}$ as follows:

$$
\begin{aligned}
\pi_{i, h}^{e}= & \left\{\left[P\left(q_{i h}^{e}+q_{i k}^{e}, q_{j h}^{e}+q_{j k}^{e}\right)-c\right] q_{i h}^{e}\right. \\
& \left.+\left[P\left(q_{j h}^{e}+q_{j k}^{e}, q_{i h}^{e}+q_{i k}^{e}\right)-c\right] q_{j h}^{e}+\delta_{j k}^{e}\left[\tau_{j k}^{e}\left(q_{j k}^{e}\right)-c q_{j k}^{e}\right]\right\}-\pi_{j-h}^{e} .
\end{aligned}
$$

Using (5), $\pi_{i, h}^{e} \geq \tilde{F}_{i h}$ then implies $q_{i h}^{e} \in \chi\left(q_{i k}^{e}, q_{j h}^{e}, q_{j h}^{e}\right)$.

- If instead $R_{h}$ is unintegrated, we can rewrite $\pi_{i, h}^{e}$ as follows:

$$
\begin{aligned}
\pi_{i, h}= & \left\{\left[P\left(q_{i h}^{e}+q_{i k}^{e}, \delta_{j h}^{e} q_{j h}^{e}+q_{j k}^{e}\right)-c\right] q_{i h}^{e}\right. \\
& \left.+\delta_{j h}^{e}\left[P\left(q_{j h}^{e}+q_{j k}^{e}, q_{i h}^{e}+q_{i k}^{e}\right) q_{j h}^{e}-\tau_{j h}^{e}\left(q_{j h}^{e}\right)\right]\right\}-\pi_{h}^{e} .
\end{aligned}
$$


Using (6), $\pi_{i, h}^{e} \geq \tilde{F}_{i h}$ then implies $q_{i h}^{e} \in \chi\left(q_{i k}^{e}, q_{j h}^{e}, q_{j h}^{e}\right)$.

Let us now turn to the case where the retailers are the proposers, and consider the negotiation between $R_{h}$ and an unintegrated $M_{i}$. Because returns to scale are constant, the contract that it signs with the rival retailer $R_{k}$ does not affect the profit that $M_{i}$ derives from the contract signed with $R_{h}$. Therefore, in the negotiation with $R_{h}, M_{i}$ is willing to supply any quantity $q_{i h}$ in return for any payment that covers the cost $c q_{i h}$.

Furthermore, as discussed in footnote 2 , if $M_{i}$ is indifferent between supplying different quantities to $R_{k}$, we assume that $M_{i}$ 's choice about $q_{i k}$ does not depend on the terms offered by $R_{h}$. It follows that, in equilibrium, $R_{h}$ will ask $M_{i}$ to supply a bilateral efficient quantity $q_{i h}^{\circ} \in \chi\left(q_{i k}^{\circ}, q_{j h}^{\circ}, q_{j k}^{\circ}\right)$, in return for a payment that just covers the cost.

\section{B Proof of Proposition 1}

Part $(i)$ of Proposition 1 derives from Lemma 1: As every channel $M_{i}-R_{h}$ must sign a cost-based contract, the quantities must be the same as if retailers were producing themselves the goods at unit cost $c$. From (P.1), in this symmetric duopoly with multiproduct firms, the equilibrium is unique, and thus symmetric: Both retailers sell the same quantity $q^{\circ}>0$ of both goods.

We now turn to part (ii) of Proposition 1. Consider first the case when the retailers are the proposers. From Lemma 1, all equilibria give zero profit to manufacturers. Conversely, this equilibrium outcome can be supported by both retailers offering to buy any quantity at cost, i.e., offering each manufacturer the two-part tariff $(c, 0)$. As a manufacturer is then indifferent between any quantity, selling $q^{\circ}$ to each retailer is a continuation equilibrium. It remains to check that no retailer has an incentive to deviate and offer a different contract. As manufacturers would not accept to supply a quantity below cost, by deviating to given quantities $q_{A}$ and $q_{B}$, a retailer cannot obtain more than the profit $\Pi_{R}^{\circ}\left(q_{A}, q_{B}\right)$. From (P.1), no such deviation is profitable. Alternatively, the same equilibrium outcome can be supported by both retailers offering each manufacturer the forcing contract $\left(q^{\circ}, c q^{\circ}\right)$ : Manufacturers are willing to accept this contract, and the argument just mentioned ensures that retailers have no incentives to deviation.

We now turn to the case when manufacturers are the proposers, and first establish the existence of an equilibrium that relies on two-part tariffs, in which each manufacturer signs the cost-based tariff $\left(w^{\circ}, F^{\circ}\right)=\left(c, \Delta^{\circ}\right)$ with each retailer. 
We first note that it is a continuation equilibrium for each retailer to accept both manufacturers' offers and then sell $\left(q^{\circ}, q^{\circ}\right)$ :

- If $R_{h}$ accepts both manufacturers' offers and expects its rival $R_{k}$ to sell $\left(q_{A k}, q_{B k}\right)=$ $\left(q^{\circ}, q^{\circ}\right)$, for $h \neq k \in\{1,2\}$, then $R_{h}$ 's profit, gross of the fixed fees, is equal to

$$
\Pi_{R}^{\circ}\left(q_{A}, q_{B}\right) \equiv\left[P\left(q_{A}+q^{\circ}, q_{B}+q^{\circ}\right)-c\right] q_{A}+\left[P\left(q_{B}+q^{\circ}, q_{A}+q^{\circ}\right)-c\right] q_{B} .
$$

This profit coincides with that of a firm in the multiproduct-firm Cournot duopoly; hence, from $(\mathbf{P . 1})$, it is a best response for $R_{h}$ to sell $\left(q_{A h}, q_{B h}\right)=\left(q^{\circ}, q^{\circ}\right)$. Therefore, if both retailers accept both manufacturers' offers, it is a continuation equilibrium for both retailers to sell $\left(q^{\circ}, q^{\circ}\right)$.

- To see that each retailer is willing to carry both goods, it suffices to note that the fee is such that:

$$
\begin{aligned}
F^{\circ} & =\Delta^{\circ}=\frac{\Pi^{\circ}}{2}-\max _{q}\left\{\left[P\left(q^{\circ}+q, q^{\circ}\right)-c\right] q\right\} \\
& =\Pi_{R}^{\circ}\left(q^{\circ}, q^{\circ}\right)-\max _{q} \Pi_{R}^{\circ}(q, 0) .
\end{aligned}
$$

Therefore, if its rival were to accept both offers and sell $\left(q^{\circ}, q^{\circ}\right)$, then a retailer:

- Obtains the same profit, $\pi_{R}^{\circ}$, by accepting both manufacturers' offers or only one of them: Accepting both offer yields a profit equal to

$$
\pi_{R}^{\circ}=\Pi_{R}^{\circ}\left(q^{\circ}, q^{\circ}\right)-2 F^{\circ}=\max _{q} \Pi_{R}^{\circ}(q, 0)-F^{\circ}
$$

where the right-hand side corresponds precisely to the maximal profit that the retailer could achieve by accepting only one manufacturer's offer.

- Strictly prefers securing this profit to rejecting both offers:

$$
\begin{aligned}
\frac{\pi_{R}^{\circ}}{2} & =\left[P\left(2 q^{\circ}, 2 q^{\circ}\right)-c\right] q^{\circ}-F^{\circ} \\
& =\max _{q}\left[P\left(q^{\circ}+q, q^{\circ}\right)-c\right] q-\left[P\left(2 q^{\circ}, 2 q^{\circ}\right)-c\right] q^{\circ} \\
& >\max _{q}\left[P\left(q^{\circ}+q, q^{\circ}\right)-c\right] q-\left[P\left(2 q^{\circ}, q^{\circ}\right)-c\right] q^{\circ} \\
& \geq 0 .
\end{aligned}
$$

We now show that manufacturers cannot profitably deviate from this candidate equilibrium. As the profit that a manufacturer achieves with a retailer is not affected by its relation with the 
other retailer, without loss of generality we can restrict attention to "one-sided" deviations, in which a manufacturer offers a deviating contract to one of the retailers. Furthermore, the above tariffs are profitable for the manufacturers:

$$
\begin{aligned}
F^{\circ} & =\Pi_{R}^{\circ}\left(q^{\circ}, q^{\circ}\right)-\max _{q} \Pi_{R}^{\circ}(q, 0) \\
& =\max _{q_{A}, q_{B}} \Pi_{R}^{\circ}\left(q_{A}, q_{B}\right)-\max _{q} \Pi_{R}^{\circ}(q, 0) \\
& >0,
\end{aligned}
$$

where the second equality comes from the definition of $q^{\circ}$ and the inequality comes from the fact that the second optimization problem is more constrained than the first one (and the optimal $q_{A}$ and $q_{B}$ are indeed both positive, as from (P.1) they are equal to $q^{\circ}>0$ ). It follows that a deviation cannot be profitable if it is not accepted by the retailer; and since the retailer can secure its equilibrium profit $\pi_{R}^{\circ}$ by accepting only the rival's offer, it must be the case that the deviation increases the joint profit of the manufacturer and of the retailer.

If the deviation induces the retailer to keep dealing with the other manufacturer, then the joint profit of the manufacturer and of the retailer (gross of the manufacturer's cost of supplying $q^{\circ}$ to the rival retailer, which is not affected by the deviation) cannot exceed

$$
\max _{q_{A}, q_{B}} \Pi_{R}^{\circ}\left(q_{A}, q_{B}\right)=\Pi_{R}^{\circ}\left(q^{\circ}, q^{\circ}\right)
$$

which the two parties already obtain in the candidate equilibrium. Therefore, such a deviation cannot be profitable.

If instead the deviation induces the retailer to reject the other manufacturer's offer, then the joint profit of the manufacturer and of the retailer (again gross of the manufacturer's cost of supplying $q^{\circ}$ to the rival retailer) cannot exceed

$$
\begin{aligned}
\max _{q}\left\{\left[P\left(q+q^{\circ}, q^{\circ}\right)-c\right] q\right\}+F^{\circ} & =\max _{q}\left\{\left[P\left(q+q^{\circ}, q^{\circ}\right)-c\right] q\right\}+\left[\Pi_{R}^{\circ}\left(q^{\circ}, q^{\circ}\right)-\max _{q} \Pi_{R}^{\circ}(q, 0)\right] \\
& =\Pi_{R}^{\circ}\left(q^{\circ}, q^{\circ}\right),
\end{aligned}
$$

which is again what they obtain in the candidate equilibrium. Therefore, such a deviation cannot be profitable either, which concludes the argument.

Finally, we characterize the set of equilibrium profits for the case when manufacturers are the proposers. As wary beliefs then boil down to passive beliefs, we can focus on one particular retailer $R$, taking as given that the other retailer will sell $\left(q^{\circ}, q^{\circ}\right)$. Let

$$
\rho_{R}^{\circ}\left(q_{A}, q_{B}\right) \equiv P\left(q_{A}+q^{\circ}, q_{B}+q^{\circ}\right) q_{A}+P\left(q_{B}+q^{\circ}, q_{A}+q^{\circ}\right) q_{B}
$$


denote the total revenue generated by $R$ selling $\left(q_{A}, q_{B}\right)$. From $(\mathbf{P . 1})$, the associated profit

$$
\Pi_{R}^{\circ}\left(q_{A}, q_{B}\right) \equiv \rho_{R}^{\circ}\left(q_{A}, q_{B}\right)-c\left(q_{A}+q_{B}\right)
$$

reaches its maximum at $\left(q_{A}, q_{B}\right)=\left(q^{\circ}, q^{\circ}\right)$, where it is equal to half the aggregate industry profit, $\Pi_{R}^{\circ}\left(q^{\circ}, q^{\circ}\right)=\Pi^{\circ} / 2$.

Let $\tau_{i R}^{\circ}\left(q_{i}\right)$ denote the equilibrium tariff that $M_{i}$ offers $R, \pi_{i, R}^{\circ} \equiv \tau_{i R}^{\circ}\left(q^{\circ}\right)-c q^{\circ}$ denote $M_{i}$ 's equilibrium profit from supplying $R$, and $\pi_{R}^{\circ} \equiv \Pi^{\circ} / 2-\pi_{A, R}^{\circ}-\pi_{B, R}^{\circ}$ denote $R$ 's equilibrium profit. Also, let

$$
\tilde{q}_{i R}^{\circ} \in \arg \max _{q_{i}} \rho_{R}^{\circ}\left(q_{i}, 0\right)-\tau_{i R}^{\circ}\left(q_{i}\right)
$$

denote the output level that $R$ would choose under exclusivity with $M_{i}$, and $\tilde{\pi}_{i, R}^{\circ} \equiv \tau_{i R}^{\circ}\left(\tilde{q}_{i R}^{\circ}\right)-$ $c \tilde{q}_{i R}^{\circ}$ denote $M_{i}$ 's associated profit (the corresponding profit for $R$ is thus $\left.\Pi_{R}^{\circ}\left(\tilde{q}_{i R}^{\circ}, 0\right)-\tilde{\pi}_{i, R}^{\circ}\right)$. Let

$$
\hat{\Pi}_{R}^{\circ} \equiv \max _{q} \Pi_{R}^{\circ}(q, 0)
$$

denote the maximal profit that can be generated under exclusivity, and

$$
\Delta^{\circ} \equiv \frac{\Pi^{\circ}}{2}-\hat{\Pi}_{R}^{\circ}
$$

denote the contribution of a manufacturer to the equilibrium profit. We have:

Lemma 3 Output and profit levels satisfy $\tilde{q}_{i R}^{\circ}>q^{\circ}$ and

$$
0 \leq \pi_{i, R}^{\circ} \leq \Delta^{\circ}
$$

for $i=A, B$, and

$$
\pi_{R}^{\circ}=\frac{\Pi^{\circ}}{2}-\pi_{A, R}^{\circ}-\pi_{B, R}^{\circ}=\Pi_{R}^{\circ}\left(\tilde{q}_{A R}^{\circ}, 0\right)-\tilde{\pi}_{A, R}^{\circ}=\Pi_{R}^{\circ}\left(\tilde{q}_{B R}^{\circ}, 0\right)-\tilde{\pi}_{B, R}^{\circ}>0 .
$$

Proof. We first provide bounds on equilibrium payoffs, before turning to the comparison between $\tilde{q}_{i R}^{\circ}$ and $q^{\circ}$.

By construction, we have $\pi_{i, R}^{\circ} \geq 0$ for $i=A, B$. Furthermore, if $\pi_{i, R}^{\circ}>\Delta^{\circ}=\Pi^{\circ} / 2-\hat{\Pi}_{R}^{\circ}$ for some $i \in\{A, B\}$, then the aggregate profit of $R$ and the other manufacturer $M_{j}$ (gross of the profit that $M_{j}$ makes with the other retailer), is such that:

$$
\pi_{R}^{\circ}+\pi_{j, R}^{\circ}=\frac{\Pi^{\circ}}{2}-\pi_{i, R}^{\circ}=\hat{\Pi}_{R}^{\circ}+\Delta^{\circ}-\pi_{i, R}^{\circ}<\hat{\Pi}_{R}^{\circ}
$$

But then, $M_{j}$ could profitably deviate to exclusivity by offering a forcing contract of the form $(\hat{q}, \hat{T})$, where $\hat{q} \equiv \arg \max _{q} \Pi_{R}^{\circ}(q, 0)$ denotes the bilaterally efficient output under exclusivity: 
By accepting this offer (and only that one), $R$ would generate a bilateral profit of $\hat{\Pi}_{R}^{\circ}$, and $\hat{T}$ can be adjusted so as to ensure that both $M_{j}$ and $R$ benefit from the deviation. It follows that $\pi_{A, R}^{\circ}, \pi_{B, R}^{\circ} \leq \Delta^{\circ}$, which in turn implies that the retailer obtains a positive profit:

$$
\pi_{R}^{\circ}=\frac{\Pi^{\circ}}{2}-\pi_{A, R}^{\circ}-\pi_{B, R}^{\circ} \geq \frac{\Pi^{\circ}}{2}-2\left(\frac{\Pi^{\circ}}{2}-\hat{\Pi}_{R}^{\circ}\right)=2 \hat{\Pi}_{R}^{\circ}-\frac{\Pi^{\circ}}{2}>0,
$$

where the inequality stems from the fact that, from Assumption (A.1), goods $A$ and $B$ are (imperfect) substitutes. Finally, (9) follows from the fact that, in equilibrium, $R$ must be indifferent between accepting both manufacturers' offers, or only one (either one). By construction, $R$ must weakly prefer accepting both offers to either one exclusively; but if $R$ were strictly preferring accepting both offers to accepting one manufacturer's offer only, then the other manufacturer could ask for a bigger share of the pie.

We now establish $\tilde{q}_{i R}^{\circ}>q^{\circ}$. By a revealed preference argument, we have:

$$
\begin{aligned}
\rho_{R}^{\circ}\left(\tilde{q}_{i R}^{\circ}, 0\right)-\tau_{i R}^{\circ}\left(\tilde{q}_{i R}^{\circ}\right) & \geq \rho_{R}^{\circ}\left(q^{\circ}, 0\right)-\tau_{i R}^{\circ}\left(q^{\circ}\right), \\
\rho_{R}^{\circ}\left(q^{\circ}, q^{\circ}\right)-\tau_{i R}^{\circ}\left(q^{\circ}\right) & \geq \rho_{R}^{\circ}\left(\tilde{q}_{i R}^{\circ}, q^{\circ}\right)-\tau_{i R}^{\circ}\left(\tilde{q}_{i R}^{\circ}\right),
\end{aligned}
$$

where the first inequality follows from (7) and the second one follows from the definition of $q^{\circ}$. Therefore:

$$
\begin{aligned}
& \rho_{R}^{\circ}\left(\tilde{q}_{i R}^{\circ}, 0\right)-\rho_{R}^{\circ}\left(q^{\circ}, 0\right) \geq \rho_{R}^{\circ}\left(\tilde{q}_{i R}^{\circ}, q^{\circ}\right)-\rho_{R}^{\circ}\left(q^{\circ}, q^{\circ}\right) \\
\Longleftrightarrow & \int_{q^{\circ}}^{\tilde{q}_{i R}^{\circ}} \partial_{1} \rho_{R}^{\circ}(x, 0) d x \geq \int_{q^{\circ}}^{\tilde{q}_{i R}^{\circ}} \partial_{1} \rho_{R}^{\circ}\left(x, q^{\circ}\right) d x \\
\Longleftrightarrow & \int_{q^{\circ}}^{\tilde{q}_{i R}^{\circ}} \int_{0}^{q^{\circ}} \partial_{12}^{2} \rho_{R}^{\circ}(x, y) d x d y \leq 0 .
\end{aligned}
$$

As $q^{\circ}>0$ and $\partial_{12}^{2} \rho_{R}^{\circ}=\partial_{12}^{2} \Pi_{R}^{\circ}<0$ from (P.1), it follows that $\tilde{q}_{i R}^{\circ} \geq q^{\circ}$.

Assume now that $\tilde{q}_{i R}^{\circ}=q^{\circ}$, which implies $\tau_{i R}^{\circ}\left(q^{\circ}\right)=\tau_{i R}^{\circ}\left(\tilde{q}_{i R}^{\circ}\right)$ and thus $\pi_{i, R}^{\circ}=\tilde{\pi}_{i, R}^{\circ}$; hence, from condition (9), both $M_{i}$ and $R$ are indifferent between $R$ accepting both manufacturers' equilibrium offers, or only $M_{i}$ 's offer. But then, $M_{i}$ could profitably deviate to exclusivity by offering a forcing contract of the form $(\hat{q}, \hat{T})$ : By accepting this offer (and only that one), $R$ would increase their bilateral profit from $\Pi_{R}^{\circ}\left(q^{\circ}, 0\right)$ to $\hat{\Pi}_{R}^{\circ}=\max _{q} \Pi_{R}^{\circ}(q, 0)>\Pi_{R}^{\circ}\left(q^{\circ}, 0\right)$, where the strict inequality stems from that fact that, from (P.1) the goods are internal strategic substitutes, and thus $\hat{q}=\arg \max _{q} \Pi_{R}^{\circ}(q, 0)>q^{\circ}$; this increase in the bilateral profit can then be shared by an appropriate $\hat{T}$ so as to ensure that both $M_{i}$ and $R$ benefit from the deviation. Therefore, $\tilde{q}_{i R}^{\circ}>q^{\circ}$.

This Lemma has an interesting implication: 
Corollary 1 There is no equilibrium in which a manufacturer offers a single forcing contract.

Proof. This follows directly from Lemma 3, which implies that equilibrium contracts must offer at least two relevant options, $q^{\circ}$ and $\tilde{q}_{i R}^{\circ} \neq q^{\circ}$.

We now show that, without loss of generality, we can restrict attention to pairs of forcing contracts:

Lemma 4 Let $\left\{\tau_{A R}^{\circ}(),. \tau_{B R}^{\circ}().\right\}$ denote the tariffs signed by retailer $R$ in a given equilibrium, with associated equilibrium profits $\pi_{A, R}^{\circ}, \pi_{B, R}^{\circ}$ and $\pi_{R}^{\circ}=\Pi^{\circ} / 2-\pi_{A, R}^{\circ}-\pi_{B, R}^{\circ}$, and let $\tilde{\tau}_{i R}^{\circ} \equiv$ $\left\{\left(q^{\circ}, \tau_{i R}^{\circ}\left(q^{\circ}\right)\right),\left(\tilde{q}_{i R}^{\circ}, \tau_{i R}^{\circ}\left(\tilde{q}_{i R}^{\circ}\right)\right)\right\}$ denote the corresponding pair of forcing contracts, respectively based on the equilibrium output level $q^{\circ}$ and on the output level $\tilde{q}_{i R}^{\circ}$ that $R$ would choose under exclusivity with $M_{i}$. Then there exists an equilibrium in which each $M_{i}$ offers the tariff $\tilde{\tau}_{i R}^{\circ}$, leading $R$ to pick the forcing contract $\left(q^{\circ}, \tau_{i R}^{\circ}\left(q^{\circ}\right)\right)$; this alternative equilibrium moreover yields the same profits $\pi_{A, R}^{\circ}, \pi_{B, R}^{\circ}$ and $\pi_{R}^{\circ}$.

Proof. Fix a given equilibrium based on tariffs $\left\{\tau_{A R}^{\circ}(),. \tau_{B R}^{\circ}().\right\}$, and consider an alternative candidate equilibrium in which each manufacturer offers instead $\tilde{\tau}_{i R}^{\circ}=\left\{\left(q^{\circ}, \tau_{i R}^{\circ}\left(q^{\circ}\right)\right),\left(\tilde{q}_{i R}^{\circ}, \tau_{i R}^{\circ}\left(\tilde{q}_{i R}^{\circ}\right)\right)\right\}$. From Lemma $3, R$ is willing to accept both offers, in which case it chooses the "option" $\left(q^{\circ}, \tau_{i R}^{\circ}\left(q^{\circ}\right)\right)$ from each $\tilde{\tau}_{i R}^{\circ}$, and is indifferent between doing so and accepting only $M_{i}$ 's offer (for $i=A, B)$, in which case it chooses the option $\left(\tilde{q}_{i R}^{\circ}, \tau_{i R}^{\circ}\left(\tilde{q}_{i R}^{\circ}\right)\right)$. We now show that manufacturers have no incentive to deviate.

Without loss of generality, we can restrict attention to deviations involving a single forcing contract. As $\pi_{i, R}^{\circ} \geq 0$ from Lemma 3, to be profitable the deviant offer must be accepted, either alone or in combination with one of the two options offered by $M_{j}$; but as these options are also available in $\tau_{j R}^{\circ}($.$) , the deviant offer would also be accepted in the original equilibrium, and$ thus cannot be profitable.

From now on, without loss of generality we will consider equilibria in which each $M_{i}$ offers two options: that is, $\tau_{i R}^{\circ}=\left\{\left(q^{\circ}, T_{i R}^{\circ}\right),\left(\tilde{q}_{i R}^{\circ}, \tilde{T}_{i R}^{\circ}\right)\right\}$.

Lemma 5 The contracts $\left(\tau_{i}^{\circ}=\left\{\left(q^{\circ}, T_{i R}^{\circ}\right),\left(\tilde{q}_{i R}^{\circ}, \tilde{T}_{i R}^{\circ}\right)\right\}\right)_{i=A, B}$ support an equilibrium if and only if the associated profits $\left(\pi_{i, R}^{\circ}=T_{i R}^{\circ}-c q^{\circ}, \tilde{\pi}_{i, R}^{\circ}=\tilde{T}_{i R}^{\circ}-c \tilde{q}_{i R}^{\circ}\right)_{i=A, B}$ and $\pi_{R}^{\circ}=\Pi^{\circ} / 2-\pi_{A, R}^{\circ}-$ $\pi_{B, R}^{\circ}$ satisfy (8), (9) and

$$
\pi_{i, R}^{\circ}-\tilde{\pi}_{i, R}^{\circ} \leq \frac{\Pi^{\circ}}{2}-\Pi_{R}^{r}\left(\tilde{q}_{i R}^{\circ}\right)
$$

where

$$
\Pi_{R}^{r}\left(q_{i}\right) \equiv \max _{q} \Pi_{R}^{\circ}\left(q, q_{i}\right)
$$


denotes the maximal aggregate profit that $R$ can generate, conditional on selling $q_{i}$ units of good $i$.

Proof. We first establish that the contracts $\left\{\tau_{A}^{\circ}, \tau_{B}^{\circ}\right\}$ support an equilibrium under conditions (8), (9) and (10).

We start by checking that $R$ is willing to accept both contracts, and to pick the options $\left\{\left(q^{\circ}, T_{A R}^{\circ}\right),\left(q^{\circ}, T_{B R}^{\circ}\right)\right\}:$

- From (9), $R$ is willing to accept the offers, and is indifferent between accepting $\left\{\left(q^{\circ}, T_{A R}^{\circ}\right),\left(q^{\circ}, T_{B R}^{\circ}\right)\right\}$, $\left\{\left(\tilde{q}_{A R}^{\circ}, \tilde{T}_{A R}^{\circ}\right)\right\}$, or $\left\{\left(\tilde{q}_{B R}^{\circ}, \tilde{T}_{B R}^{\circ}\right)\right\}$;

- In addition, $R$ prefers accepting $\left\{\left(q^{\circ}, T_{A R}^{\circ}\right),\left(q^{\circ}, T_{B R}^{\circ}\right)\right\}$, which yield $\pi_{R}^{\circ}$, to accepting only $\left(q^{\circ}, T_{i R}^{\circ}\right)$ : This amounts to

$$
\frac{\Pi^{\circ}}{2}-\pi_{A, R}^{\circ}-\pi_{B, R}^{\circ}>\Pi_{R}^{\circ}\left(q^{\circ}, 0\right)-\pi_{i, R}^{\circ}
$$

or:

$$
\pi_{j, R}^{\circ}<\frac{\Pi^{\circ}}{2}-\Pi_{R}^{\circ}\left(q^{\circ}, 0\right),
$$

which follows from (8), as the RHS of (11) is strictly larger than $\Delta^{\circ}$.

- From (7), if $R$ were to accept $M_{i}$ 's contract only, then it would pick the option $\left(\tilde{q}_{i R}^{\circ}, \tilde{T}_{i R}^{\circ}\right)$ rather than $\left(q^{\circ}, T_{i R}^{\circ}\right)$.

- Finally, $R$ prefers accepting $\left\{\left(q^{\circ}, T_{A R}^{\circ}\right),\left(q^{\circ}, T_{B R}^{\circ}\right)\right\}$ to accepting $\left\{\left(\tilde{q}_{i}^{\circ}, \tilde{T}_{i R}^{\circ}\right),\left(q^{\circ}, T_{j R}^{\circ}\right)\right\}$, which amounts to:

$$
\begin{aligned}
& \frac{\Pi^{\circ}}{2}-\pi_{A, R}^{\circ}-\pi_{A, R}^{\circ} \geq \Pi_{R}^{\circ}\left(q^{\circ}, \tilde{q}_{i R}^{\circ}\right)-\pi_{j, R}^{\circ}-\tilde{\pi}_{i, R}^{\circ} \\
& \Longleftrightarrow \pi_{i, R}^{\circ}-\tilde{\pi}_{i, R}^{\circ} \leq \frac{\Pi^{\circ}}{2}-\Pi_{R}^{\circ}\left(q^{\circ}, \tilde{q}_{i R}^{\circ}\right),
\end{aligned}
$$

and is thus implied by (10).

We now turn to deviations by the manufacturers:

- $M_{i}$ has no incentive to deviate by making an unacceptable offer (or no offer), as $\pi_{i, R}^{\circ} \geq 0$.

- $M_{i}$ has no incentive to deviate to exclusivity. To see that, it suffices to note that, as $R$ can secure its equilibrium profit by accepting $M_{j}$ 's offer only, to be profitable a deviation must increase the joint profit of $M_{i}$ and $R$; but along the equilibrium path, this joint profit (gross of the profit that $M_{i}$ makes with the other retailer) satisfies:

$$
\pi_{R}^{\circ}+\pi_{i, R}^{\circ}=\frac{\Pi^{\circ}}{2}-\pi_{j, R}^{\circ} \geq \frac{\Pi^{\circ}}{2}-\Delta^{\circ}=\hat{\Pi}_{R}^{\circ}
$$

where $\hat{\Pi}_{R}^{\circ}$ is the maximal profit that can be achieved under exclusivity. 
- $M_{i}$ cannot profitably deviate by inducing $R$ to combine the deviant offer with $M_{j}$ 's equilibrium option $\left(q^{\circ}, T_{j R}^{\circ}\right)$. As the profit generated by $R$ is maximal along the equilibrium path (that is, $\Pi^{\circ} / 2=\max _{q_{A}, q_{B}} \Pi_{R}^{\circ}\left(q_{A}, q_{B}\right)$ ), a deviation by $M_{i}$ that induces $R$ to combine the deviant offer with $M_{j}$ 's equilibrium option $\left(q^{\circ}, T_{j R}^{\circ}\right)$ cannot be profitable, as this would maintain $M_{j}$ 's profit at the equilibrium level (that is, $M_{j}$ would obtain a $T_{j R}^{\circ}-c q^{\circ}=\pi_{j, R}^{\circ}$ ), and a deviation cannot lower $R$ 's profit either.

- Finally, $M_{i}$ cannot profitably deviate by inducing $R$ to combine the deviant offer with $M_{j}$ 's alternative option $\left(\tilde{q}_{j}^{\circ}, \tilde{T}_{j R}^{\circ}\right)$. As $R$ can secure its equilibrium profit by accepting $M_{j}$ 's offer only, such a deviation could only be profitable if it increased the joint profit of $M_{i}$ and $R$, gross of the profit that $M_{i}$ makes with the other retailer, that is, only if :

$$
\Pi_{R}^{\circ}\left(q_{i}, \tilde{q}_{j}^{\circ}\right)-\tilde{\pi}_{j, R}^{\circ}>\frac{\Pi^{\circ}}{2}-\pi_{j, R}^{\circ},
$$

which is ruled out by (10) (written for $M_{j}$ ).

Conversely, conditions (8) and (9) are necessary by Lemma 3. Moreover, the above analysis shows that, if (10) were violated, then $M_{j}$ could profitably deviate by inducing $R$ to combine $M_{i}$ 's alternative option $\left(\tilde{q}_{i}^{\circ}, \tilde{T}_{i R}^{\circ}\right)$ with a forcing contract based on $q_{R}^{r}\left(\tilde{q}_{i}^{\circ}\right)=\arg \max _{q} \Pi_{R}^{\circ}\left(q, \tilde{q}_{i}^{\circ}\right)$ and a payment giving $R$ slightly more than its equilibrium profit.

We now show that $M_{j}$ 's equilibrium profit can cover the full range $\left[0, \Delta^{\circ}\right]$ by relying on a "large enough" quantity $\tilde{q}_{i R}^{\circ}$ for $M_{i}$ 's exclusive deal option.

Lemma 6 For any $\pi_{A, R}^{\circ}, \pi_{B, R}^{\circ} \in\left[0, \Delta^{\circ}\right]$, there exists an equilibrium yielding profits $\pi_{A, R}^{\circ}, \pi_{B, R}^{\circ}$ and $\pi_{R}^{\circ}=\Pi^{\circ} / 2-\pi_{A, R}^{\circ}-\pi_{B, R}^{\circ}$.

Proof. We first note that the expression $\Pi_{R}^{r}(\tilde{q})-\Pi_{R}^{\circ}(0, \tilde{q})=\max _{q} \Pi_{R}^{\circ}(q, \tilde{q})-\Pi_{R}^{\circ}(0, \tilde{q})$ decreases as $\tilde{q}$ increases. Using the envelope theorem, and letting $q_{R}^{r}(\tilde{q})=\arg \max _{q} \Pi_{R}^{\circ}(q, \tilde{q})$ denote $R$ 's "best response" to selling a quantity $\tilde{q}$ of the other brand, we have:

$$
\begin{aligned}
\frac{d}{d \tilde{q}}\left[\Pi_{R}^{r}(\tilde{q})-\Pi_{R}^{\circ}(0, \tilde{q})\right] & =\partial_{2} \Pi_{R}^{\circ}\left(q_{R}^{r}(\tilde{q}), \tilde{q}\right)-\partial_{2} \Pi_{R}^{\circ}(0, \tilde{q}) \\
& =\int_{0}^{q_{R}^{r}(\tilde{q})} \partial_{12} \Pi_{R}^{\circ}(q, \tilde{q}) d q,
\end{aligned}
$$

which is negative as long as $q_{R}^{r}(\tilde{q})>0$, as $\partial_{12} \Pi_{R}^{\circ}<0$ by (P.1). From $(\mathbf{A . 2}), q_{R}^{r}(\tilde{q})=0$ for $\tilde{q}$ large enough; let $\bar{q}$ denote such a quantity and, for any $\pi_{A, R}^{\circ}, \pi_{A, R}^{\circ} \in\left[0, \Delta^{\circ}\right]$, consider the pairs of forcing contracts $\left\{\left(q^{\circ}, T_{A R}^{\circ}\right),\left(\bar{q}, \tilde{T}_{R}^{\circ}\right)\right\}$ and $\left\{\left(q^{\circ}, T_{B R}^{\circ}\right),\left(\bar{q}, \tilde{T}_{R}^{\circ}\right)\right\}$, where the payments are such that $T_{i R}^{\circ}=c q^{\circ}+\pi_{i, R}^{\circ}$, for $i=A, B$, and:

$$
\tilde{T}_{R}^{\circ}=c \bar{q}+\Pi_{R}^{\circ}(0, \bar{q})+\pi_{A, R}^{\circ}+\pi_{B, R}^{\circ}-\frac{\Pi^{\circ}}{2} .
$$


By construction, the profits associated with these contracts satisfy conditions (8) and (9). Furthermore, the remaining equilibrium condition (10) is also satisfied:

$$
\begin{aligned}
\pi_{j, R}^{\circ} & \geq \Pi_{R}^{r}(\bar{q})-\Pi_{R}(0, \bar{q})=0 \\
\Longrightarrow \frac{\Pi^{\circ}}{2}-\Pi_{R}^{r}(\bar{q}) & \geq \frac{\Pi^{\circ}}{2}-\Pi_{R}^{\circ}(0, \bar{q})-\pi_{j, R}^{\circ} \\
& =\pi_{i, R}^{\circ}+\frac{\Pi^{\circ}}{2}-\left(\Pi_{R}^{\circ}(0, \bar{q})+\pi_{A, R}^{\circ}+\pi_{B, R}^{\circ}\right) \\
& =\pi_{i, R}^{\circ}-\tilde{\pi}_{i, R}^{\circ} .
\end{aligned}
$$

Therefore, from Lemma 5, these pairs of forcing contracts support an equilibrium, with profits $\left(\pi_{A, R}^{\circ}, \pi_{B, R}^{\circ}\right)$ for the manufacturers and $\pi_{R}^{\circ}=\Pi^{\circ} / 2-\pi_{A, R}^{\circ}-\pi_{B, R}^{\circ}$ for $R$.

Finally, we have:

Lemma 7 If $\pi_{i, R}^{\circ}<\Delta^{\circ}$, then $M_{j}$ strictly prefers $R$ to accept both equilibrium offers than only its own equilibrium offer (i.e., $\tilde{\pi}_{j, R}^{\circ}<\pi_{j, R}^{\circ}$ ).

Proof. Note first that, as $M_{j}$ could induce $R$ to switch to exclusivity by slightly reducing $\tilde{T}_{i R}^{\circ}$, we must therefore have $\pi_{i, R}^{\circ} \geq \tilde{\pi}_{i, R}^{\circ}$. Furthermore, if $\pi_{i, R}^{\circ}<\Delta^{\circ}$, then condition (9) yields:

$$
\begin{aligned}
\pi_{j, R}^{\circ}-\tilde{\pi}_{j, R}^{\circ} & =\frac{\Pi^{\circ}}{2}-\pi_{i, R}^{\circ}-\Pi_{R}^{\circ}\left(\tilde{q}_{j R}^{\circ}, 0\right) \\
& >\max _{\tilde{q}} \Pi_{R}^{\circ}(\tilde{q}, 0)-\Pi_{R}^{\circ}\left(\tilde{q}_{j R}^{\circ}, 0\right) \\
& \geq 0 .
\end{aligned}
$$

As manufacturers obtain zero profit when retailers are the proposers, it follows from Lemma 3 that the set of expected profits for the two manufacturers is $\left[0,2 \alpha \Delta^{\circ}\right] \times\left[0,2 \alpha \Delta^{\circ}\right]$. Furthermore, if $M_{i}$ 's expected profit is less than $2 \alpha \Delta^{\circ}$, then from Lemma 3 it must be the case that $\pi_{i, R}^{\circ}<\Delta^{\circ}$ for some retailer $R$; but then, it follows from Lemma 7 that $M_{j}$ must offer to supply the incremental quantity $\tilde{q}_{j R}^{\circ}-q^{\circ}$ below cost.

\section{Proof of Proposition 2}

Part $(i)$ of Proposition 2 derives from Lemma 1: As $R_{1}$ must sign a cost-based contract with $M_{A}$, and $R_{2}$ must sign a cost-based contract with both manufacturers, the quantities must be the same as if $R_{1}$ could produce itself good $A$ at unit cost $c$, and $R_{2}$ could produce both goods at the same cost $c$. From (P.1), the equilibrium of this asymmetric duopoly is unique. 
It follows that prices and quantities, and thus the profits generated by the two retailers, are the same in all equilibria:

$$
\Pi_{1}^{*}=\left(p_{A}^{*}-c\right) q_{A 1}^{*}+\left(p_{B}^{*}-c\right) q_{B 1}^{*} \text { and } \Pi_{2}^{*}=\left(p_{B}^{*}-c\right) q_{B 2}^{*},
$$

where $p_{i}^{*}=P\left(Q_{i}^{*}, Q_{j}^{*}\right)$ is the equilibrium price of $\operatorname{good} i=A, B$.

We now turn to part (ii) of Proposition 2. When the retailers are the proposers, the same logic as before applies. From Lemma 1, all equilibria again give zero profit to manufacturers, and conversely, this equilibrium outcome can be supported by both retailers offering to buy any quantity at cost, i.e., $R_{1}$ offers each manufacturer the two-part tariff $(c, 0)$, and $R_{2}$ offers $M_{B}$ the same tariff. As manufacturers are then indifferent about quantity choices, the quantity profile $\left(q_{A 1}^{*}, q_{B 1}^{*}, q_{B 2}^{*}\right)$ is a continuation equilibrium. It remains to check that no retailer has an incentive to deviate and offer a different contract. As manufacturers would not accept to supply a quantity below cost, by deviating to some quantities $q_{A}$ and $q_{B}, R_{1}$ cannot obtain more than:

$$
\Pi_{1}\left(q_{A}, q_{B} ; 0, q_{B 2}^{*}\right)=\left[P\left(q_{A 1}, q_{B 1}+q_{B 2}^{*}\right)-c\right] q_{A 1}+\left[P\left(q_{B 1}+q_{B 2}^{*}, q_{A 1}\right)-c\right] q_{B 1},
$$

and likewise by deviating to some quantity $q_{B 2}$ and $R_{2}$ cannot obtain more than:

$$
\Pi_{2}\left(0, q_{B 2} ; q_{A 1}^{*}, q_{B 1}^{*}\right)=\left[P\left(q_{B 1}^{*}+q_{B 2}, q_{A 1}^{*}\right)-c\right] q_{B 1} .
$$

But these are the profits that the two firms could respectively obtain by deviating from the equilibrium $\left(q_{A 1}^{*}, q_{B 1}^{*}, q_{B 2}^{*}\right)$ in the asymmetric Cournot duopoly where one firm produces both goods (at cost $c$ ), and the other firm only produces good $B$ (at the same cost). From (P.1), no such deviation is profitable.

Consider now the case when the manufacturers are the proposers. We first establish the existence of an equilibrium in which they offer cost-based two-part tariffs of the form $\left(w_{i, h}^{*}, F_{i, h}^{*}\right)=$ $\left(c, \Delta_{i, h}^{*}\right)$, where $\Delta_{i, h}^{*}$ denotes $M_{i}$ 's contribution to the profit generated by $R_{h}$, namely (as $M_{B}$ is the sole supplier of $R_{2}$, its "contribution" amounts to $R_{2}$ 's profit is $\Pi_{2}^{*}$ ):

$$
\begin{aligned}
& \Delta_{A, 1}^{*}=\Pi_{1}^{*}-\max _{q_{B 1}}\left\{\left[P\left(q_{B 1}+q_{B 2}^{*}, 0\right)-c\right] q_{B 1}\right\}, \\
& \Delta_{B, 1}^{*}=\Pi_{1}^{*}-\max _{q_{A 1}}\left\{\left[P\left(q_{A 1}, q_{B 2}^{*}\right)-c\right] q_{A 1}\right\}, \\
& \Delta_{B, 2}^{*}=\Pi_{2}^{*}=\left(p_{B}^{*}-c\right) q_{B 2}^{*} .
\end{aligned}
$$

From (P.1), it is then a continuation equilibrium for $R_{1}$ to sell $\left(q_{A 1}, q_{B 1}\right)=\left(q_{A 1}^{*}, q_{B 1}^{*}\right)$ and for $R_{2}$ to sell $q_{B 2}=q_{B 2}^{*}$. Next, we note that each retailer is willing to accept all offers made. Indeed, the fees are such that: 
- If $R_{1}$ expects $R_{2}$ to sell $q_{B 2}^{*}$, then $R_{1}$ is indifferent between accepting both manufacturers' offers or only one (either one);

- If $R_{2}$ expects $R_{1}$ to sell $\left(q_{A 1}^{*}, q_{B 1}^{*}\right)$, then $R_{2}$ is indifferent between accepting $M_{B}$ 's offer or not.

It thus suffices to check that $R_{1}$ is strictly better-off accepting the manufacturers' offers rather than rejecting both of them; indeed, we have:

$$
\begin{aligned}
\pi_{1}^{*}= & \left(p_{A}^{*}-c\right) q_{A 1}^{*}+\left(p_{B}^{*}-c\right) q_{B 1}^{*}-\Delta_{A, 1}^{*}-\Delta_{B, 1}^{*} \\
= & \max _{q_{A 1}}\left\{\left[P\left(q_{A 1}, q_{B 2}^{*}\right)-c\right] q_{A 1}\right\}+\max _{q_{B 1}}\left\{\left[P\left(q_{B 1}+q_{B 2}^{*}, 0\right)-c\right] q_{B 1}\right\} \\
& -\left[\left(p_{A}^{*}-c\right) q_{A 1}^{*}+\left(p_{B}^{*}-c\right) q_{B 1}^{*}\right] \\
= & \max _{q_{A 1}}\left\{\left[P\left(q_{A 1}, q_{B 2}^{*}\right)-c\right] q_{A 1}\right\}-\left[P\left(q_{A 1}^{*}, q_{B 1}^{*}+q_{B 2}^{*}\right)-c\right] q_{A 1}^{*} \\
& +\max _{q_{B 1}}\left\{\left[P\left(q_{B 1}+q_{B 2}^{*}, 0\right)-c\right] q_{B 1}\right\}-\left[P\left(q_{B 1}^{*}+q_{B 2}^{*}, q_{A 1}^{*}\right)-c\right] q_{B 1}^{*} \\
> & \max _{q_{A 1}}\left\{\left[P\left(q_{A 1}, q_{B 2}^{*}\right)-c\right] q_{A 1}\right\}-\left[P\left(q_{A 1}^{*}, q_{B 2}^{*}\right)-c\right] q_{A 1}^{*} \\
& +\max _{q_{B 1}}\left\{\left[P\left(q_{B 1}+q_{B 2}^{*}, 0\right)-c\right] q_{B 1}\right\}-\left[P\left(q_{B 1}^{*}+q_{B 2}^{*}, 0\right)-c\right] q_{B 1}^{*} \\
\geq & 0 .
\end{aligned}
$$

Thus, if these contracts are offered, it is a continuation equilibrium for retailers to accept all offers, and then to sell the quantities $\left(q_{A 1}^{*}, q_{B 1}^{*}, q_{B 2}^{*}\right)$. We now show that manufacturers cannot profitably deviate from this candidate equilibrium.

We first note that the above tariffs are profitable for the manufacturers, as each manufacturer contributes positively to the profits generated by the retailers. ${ }^{42}$ It follows that a deviation cannot be profitable if it is not accepted by the retailer. But then, $M_{B}$ cannot profitably deviate in its offer to $R_{2}$, as it already appropriates all the profit that $R_{2}$ can expect to generate. Likewise, no $M_{i}$ can profitably deviate in its dealing with $R_{1}$, as: (i) $M_{i}$ and $R_{1}$ cannot increase their joint profit above the equilibrium level, as $M_{j}$ does not obtain more than its contribution to the profit generated by $R_{1}$; and (ii) following a deviation by $M_{i}, R_{1}$ can still secure its equilibrium profit by accepting only $M_{j}$ 's offer.

\footnotetext{
${ }^{42}$ For instance, $\Delta_{A, 1}^{*}$ can be expressed as$$
\begin{aligned}
\Delta_{A, 1}^{*} & =\left\{\left[P\left(q_{A 1}^{*}, q_{B 1}^{*}+q_{B 2}^{*}\right)-c\right] q_{A 1}^{*}+\left[P\left(q_{B 1}^{*}+q_{B 2}^{*}, 0\right)-c\right] q_{B 1}^{*}\right\}-\max _{q_{B 1}}\left\{\left[P\left(q_{B 1}+q_{B 2}^{*}, 0\right)-c\right] q_{B 1}\right\} \\
& =\max _{q_{A 1}, q_{B 1}}\left\{\left[P\left(q_{A 1}, q_{B 1}+q_{B 2}^{*}\right)-c\right] q_{A 1}+\left[P\left(q_{B 1}+q_{B 2}^{*}, 0\right)-c\right] q_{B 1}\right\}-\max _{q_{B 1}}\left\{\left[P\left(q_{B 1}+q_{B 2}^{*}, 0\right)-c\right] q_{B 1}\right\},
\end{aligned}
$$

which is positive as $q_{A 1}^{*}>0$. 
This establishes the existence of an equilibrium that relies on cost-based two-part tariffs, and gives $M_{A}$ and $M_{B}$ equilibrium expected profits equal to $\alpha \Delta_{A, 1}^{*}$ and $\alpha\left(\Delta_{B, 1}^{*}+\Delta_{B, 2}^{*}\right)=$ $\alpha\left(\Pi_{2}^{*}+\Delta_{B, 1}^{*}\right)$. Finally, going through the same steps of in the proof of Proposition 1, it can be shown that any expected payoffs in the set $\left[0, \alpha \Delta_{A, 1}^{*}\right] \times\left[\alpha \Pi_{2}^{*}, \alpha\left(\Pi_{2}^{*}+\Delta_{B, 1}^{*}\right)\right]$ can be supported in equilibrium. In particular, when manufacturers are the proposers: (i) attention can be restricted to manufacturers offering a pair of forcing contracts to $R_{1}$, and a single forcing contract to $R_{2}$; (ii) $R_{1}$ must be indifferent between accepting both manufacturers' offers and accepting only one of them (either one), and $R_{2}$ must be indifferent between accepting $M_{B}$ 's offer or not.

\section{Proof of Proposition 3}

Part $(i)$ of Proposition 3 follows again from Lemma 1. In addition, as each firm is locked into an exclusive relationship with a single partner, the proposers always appropriate all the profit generated by their channel. That is, when the manufacturers are the proposers, each $M_{i}$ fully appropriates the profit generated by good $i$; when instead the retailers are the proposers, $R_{1}$ and $R_{2}$ fully appropriate the profits generated by, respectively, goods $A$ and $B$.

We now turn to part $(i i)$ of Proposition 3. Suppose first that the manufacturers are the proposers, and consider a candidate equilibrium in which they both offer the cost-based twopart tariff $\left(w^{* *}, F^{* *}\right)=\left(c, \Pi_{R}^{* *}\right)$, where

$$
\Pi_{R}^{* *}=\left[P\left(Q^{* *}, Q^{* *}\right)-c\right] Q^{* *}=\frac{\Pi^{* *}}{2}
$$

denotes the profit generated by a retailer. The retailers are willing to accept those contracts, in which case they each put $Q^{* *}$ on the market and break even. Furthermore, each manufacturer obtains all the profits generated by its good, which is moreover maximal given the output level $Q^{* *}$ of the other good; it follows that there is no profitable deviation.

When instead the retailers are the proposers, a similar argument implies that there exists an equilibrium in which retailers offer the cost-based two-part tariff $(c, 0)$, and then each manufacturer chooses to sell $Q^{* *}$.

\section{E Proof of Proposition 4}

We start with part $(i)$ of Proposition 4. Let $\Pi^{\circ}, \Pi^{*}$, and $\Pi^{* *}$ denote the equilibrium industry profit under no exclusive dealing, single exclusive dealing, and pairwise exclusive dealing, respectively. From $(\mathbf{P . 2})$, we have: $\Pi^{\circ}<\Pi^{*}<\Pi^{* *}$. 
In the absence of exclusive dealing, at least one pair, say $M_{A}-R_{1}$, makes a weakly lower joint profit than the other pair, i.e., $\Pi_{M_{A}-R_{1}}^{\circ} \leq \Pi^{\circ} / 2 \leq \Pi_{M_{B}-R_{2}}^{\circ}$. We show below that this pair, $M_{A}-R_{1}$, would benefit from $M_{A}$ dealing exclusively with $R_{1}$, although less so if $M_{B}$ also deals exclusively with $R_{2}$; i.e.:

$$
\Pi_{M_{A}-R_{1}}^{\circ}<\Pi_{M_{A}-R_{1}}^{* *}<\Pi_{M_{A}-R_{1}}^{*},
$$

where $\Pi_{M_{A}-R_{1}}^{\circ}, \Pi_{M_{A}-R_{1}}^{*}$ and $\Pi_{M_{A}-R_{1}}^{* *}$ denote the equilibrium joint profit of the pair $M_{A}-R_{1}$ under, respectively: (i) no exclusivity; (ii) single exclusive dealing where $M_{A}$ only supplies $R_{1}$ but not $R_{2}$, whereas $M_{B}$ supplies both retailers; and (iii) pairwise exclusive dealing where $M_{A}$ supplies $R_{1}$ only, and $M_{B}$ supplies $R_{2}$ only.

The first inequality in (12) follows from (P.2), which yields

$$
\Pi_{M_{A}-R_{1}}^{* *}=\frac{\Pi^{* *}}{2}>\frac{\Pi^{\circ}}{2} \geq \Pi_{M_{A}-R_{1}}^{\circ} .
$$

To establish the second inequality in (12) we first note that, under single exclusivity, $M_{A}$ and $R_{1}$ must jointly obtain at least what they could get by deviating to pairwise exclusivity, that is:

$$
\Pi_{M_{A}-R_{1}}^{*} \geq \max _{q_{A 1}}\left[P\left(q_{A 1}, q_{B 2}^{*}\right)-c\right] q_{A 1}
$$

To see this, it suffices to note that $M_{A}$ or $R_{1}$ (depending on whether manufacturers or retailers make offers) could otherwise profitably deviate to a forcing contract $\left(\hat{q}_{A 1}, \hat{T}_{A 1}\right)$, where

$$
\hat{q}_{A 1} \equiv \arg \max _{q_{A 1}}\left[P\left(q_{A 1}, q_{B 2}^{*}\right)-c\right] q_{A 1}
$$

This would increase the joint profit of $M_{A}$ and $R_{1}$, and the fixed fee $\hat{T}_{A 1}$ could be adjusted so as to increase both parties' profits, thereby ensuring that the offer will be accepted, and that the deviation is profitable. ${ }^{43}$

This, in turn, implies that the pair $M_{A}-R_{1}$ obtains indeed more under single exclusivity than under pairwise exclusivity:

$$
\begin{aligned}
\Pi_{M_{A}-R_{1}}^{*} & \geq \max _{q_{A 1}}\left[P\left(q_{A 1}, q_{B 2}^{*}\right)-c\right] q_{A 1} \\
& >\max _{q_{A 1}}\left[P\left(q_{A 1}, q_{B 2}^{* *}\right)-c\right] q_{A 1}=\Pi_{M_{A}-R_{1}}^{* *}
\end{aligned}
$$

where the second inequality follows from (A.1) and (P.2), which implies $q_{B 2}^{*}<q_{B 2}^{* *}$.

\footnotetext{
${ }^{43}$ When the manufacturers are the proposers, $R_{1}$ may find it profitable to combine $M_{A}$ 's deviant offer with the equilibrium contract offered by $M_{B}$. If so, this can only increase $R_{1}$ 's incentive to accept $M_{A}$ 's deviant offer, without affecting $M_{A}$ 's deviation profit. (The issue does not arise when the retailers are the proposers, as by assumption an exclusive dealing provision prevents $M_{A}$ from dealing with $R_{2}$.)
} 
We now turn to part (ii) of Proposition 4. Using again (P.2), we have:

$$
\Pi_{M_{B}-R_{2}}^{* *}=\frac{\Pi^{* *}}{2}>\frac{\Pi^{*}}{2}>\Pi^{*}-\Pi_{M_{A}-R_{1}}^{*}=\Pi_{M_{B}-R_{2}}^{*},
$$

where the second inequality follows from

$$
\Pi_{M_{A}-R_{1}}^{*}>\Pi_{M_{A}-R_{1}}^{* *}=\frac{\Pi^{* *}}{2}>\frac{\Pi^{*}}{2} .
$$

Hence, when $M_{A}$ deals exclusively with $R_{1}, M_{B}$ and $R_{2}$ obtain a larger joint profit if they, too, engage in exclusive dealing.

\section{F Proof of Proposition 5}

We study here the adoption game introduced in Section 5.2. To fix ideas, we assume that $M_{i}$ and $R_{h}$ get the opportunity of negotiating an exclusivity provision in the first stage, followed by $M_{j}$ and $R_{k}$ in the second stage, where $i \neq j \in\{A, B\}$ and $h \neq k \in\{1,2\}$.

We first note that a single player gets to move at every node of the game; hence, by construction, an equilibrium exists.

Second, in order to determine which agreements the negotiating firms will sign at any given stage, it suffices to keep track of the impact that these agreements will eventually have on the negotiating parties' joint profit:

- This is obvious in stage 2, regardless of whether manufacturers or retailers are the proposers, as $M_{j}$ or $R_{k}$ can use a lump-sum transfer to share their joint profit in any way they want.

- This also holds in stage 1 , as the agreement signed by $M_{i}$ and $R_{h}$ (or the lack thereof) affects the joint profit of $M_{i}$ and $R_{h}$ only through the influence that their own agreement may have on the subsequent agreement signed by $M_{j}$ or $R_{k}$ in stage 2 ; in particular, $M_{i}$ and $R_{h}$ cannot use their own agreement to improve their "bargaining position" in the subsequent negotiation, as they are not involved in that negotiation (also, by construction, any lump-sum transfer agreed upon in stage 1 has no impact on the joint profit eventually obtained by $M_{i}$ and $R_{h}$ at the end of stage 2 ).

Third, in stage $1, M_{i}$ and $R_{h}$ can secure a joint profit equal to $\Pi^{* *} / 2$ by agreeing to deal exclusively with each other (i.e., by combining exclusive distribution and single branding provisions), which de facto imposes pairwise exclusivity. They can also secure this joint profit by 
simply signing an exclusive distribution agreement, as, from part (ii) of Proposition 4, this leads $M_{j}$ and $R_{k}$ to sign an exclusive distribution agreement as well in stage 2 .

Fourth, if instead $M_{i}$ and $R_{h}$ were to sign a single branding agreement in stage 1 , then in stage $2 M_{j}$ and $R_{k}$ would not sign any exclusivity agreement, as they prefer any equilibrium under single exclusivity (where they distribute both goods) to pairwise exclusivity. This, in turn, implies that $M_{i}$ and $R_{h}$ do not sign a single branding agreement in stage 1 , as doing so would give them less profit than the joint profit $\Pi^{* *} / 2$ that they can secure, for example, by signing an exclusive distribution agreement in stage 1.

Finally, consider a candidate equilibrium in which $M_{i}$ and $R_{h}$ do not adopt any exclusivity provision in stage 1 . In stage $2, M_{j}$ and $R_{k}$ will not sign a single branding contract, neither on a stand-alone basis nor combined with an exclusive distribution agreement, as this is dominated by signing only an exclusive distribution agreement: By doing so, they end-up in a single exclusivity situation where $R_{k}$ distributes both goods, whereas $R_{h}$ distributes only $M_{i}$ 's product; from Proposition 4, they thereby obtain a larger joint profit than (i) in the other single exclusivity situation where $R_{k}$ is the retailer distributing a single product, and (ii) in the pairwise exclusivity situation (which is reached through adoption of both single branding and exclusive distribution provisions). It follows that, in stage $2, M_{j}$ and $R_{k}$ will either adopt no exclusivity provision, or sign an exclusive distribution agreement. But in both cases, the resulting outcomes give $M_{i}$ and $R_{h}$ a lower joint profit than $\Pi^{* *} / 2$, which they can secure by signing among themselves an exclusive distribution agreement in stage 1.

Summing-up, there exists an equilibrium, and all equilibria yield pairwise exclusivity - either each pair successively signs an exclusive distribution agreement, or the first pair adopts both single branding and exclusive distribution provisions.

Note that these insights apply as well when single branding is ruled out, i.e., when firms can adopt only exclusive distribution agreements. As noted above, in that case the first pair obtains a joint profit of $\Pi^{* *} / 2$ if it signs an exclusive distribution agreement in stage 1 , as this leads the other pair to do the same in stage 2. If instead the first pair does not sign an exclusive distribution agreement in stage 1 , then in stage 2 :

- Either the other pair responds by adopting no exclusivity provision as well; but this cannot be an equilibrium, as from Proposition 4, at least one pair would benefit from switching to an exclusive distribution agreement, regardless of whether the other pair does the same or not. 
- Or the other pair responds by signing an exclusive distribution agreement; but this cannot be an equilibrium either, as the first pair then obtains less than $\Pi^{* *} / 2$, which it could obtain by signing an exclusive distribution agreement in stage 1 .

\section{G Proof of Proposition 6}

As $Q^{\circ}>Q^{* *}$, to show that consumer surplus is greater in the absence of exclusive dealing, it suffices to note that $S(Q, Q)$ increases with $Q$ :

$$
\frac{d S(Q, Q)}{d Q}=-2 Q\left[\partial_{1} P(Q, Q)+\partial_{2} P(Q, Q)\right]
$$

which is positive from (A.1).

Exclusive dealing also harms welfare, as $W(Q, Q)$ increases with $Q$ as long as $P(Q, Q)>c$ :

$$
\begin{aligned}
\frac{d W(Q, Q)}{d Q} & =P(Q, Q)-c+\int_{0}^{Q} \partial_{2} P(q, Q) d q+P(Q, 0)-c \\
& =P(Q, Q)-c+\int_{0}^{Q} \partial_{2} P(Q, q) d q+P(Q, 0)-c \\
& =2[P(Q, Q)-c],
\end{aligned}
$$

where the second equality follows from the fact that demand symmetry implies that $\partial_{2} P(q, Q) \equiv$ $\partial_{2} P(Q, q)$. To conclude the argument, it suffices to note that $P(Q, Q)$ is decreasing in $Q$ from (A.1), and that $P\left(Q^{\circ}, Q^{\circ}\right)>c$.

\section{H Proof of Proposition 7}

To prove Proposition 7, consider the hypothetical Cournot duopoly where firm 1 produces both goods $A$ and $B$ at marginal cost $c$ whereas firm 2 produces good $A$ at marginal cost $\hat{c}$ and good $B$ at marginal cost $c$. The equilibrium aggregate quantities, $\left(\hat{Q}_{A}(\hat{c}), \hat{Q}_{B}(\hat{c})\right)$, are a solution to:

$$
\begin{aligned}
& \hat{Q}_{A}(\hat{c})=\hat{R}\left(\hat{Q}_{B}(\hat{c}), \hat{c}\right), \\
& \hat{Q}_{B}(\hat{c})=R\left(\hat{Q}_{A}(\hat{c})\right),
\end{aligned}
$$

where from Assumption $(B)$ the aggregate best responses $\hat{R}$ and $R$ each have a slope that lies between -1 and 0 , and the best response $\hat{R}$ moreover decreases as $\hat{c}$ increases.

Note that $\left(Q^{\circ}, Q^{\circ}\right)=\left(\hat{Q}_{A}(c), \hat{Q}_{B}(c)\right)$ and $\left(Q_{A}^{*}, Q_{B}^{*}\right)=\left(\hat{Q}_{A}\left(\hat{c}^{*}\right), \hat{Q}_{B}\left(\hat{c}^{*}\right)\right)$, where

$$
\hat{c}^{*} \equiv P\left(Q_{A}^{*}, Q_{B}^{*}\right)+\partial_{2} P\left(Q_{B}^{*}, Q_{A}^{*}\right) q_{B 2}^{*} .
$$


is the cost level which leads firm 2 to stop selling good $A$. To see this, note that, in the hypothetical Cournot duopoly: (i) firm 1 behaves as in the asymmetric duopoly setting introduced in Section 3 , and thus chooses $\left(q_{A 1}, q_{B 1}\right)=\left(q_{A 1}^{*}, q_{B 1}^{*}\right)$ in response to $\left(q_{A 2}, q_{B 2}\right)=\left(0, q_{B 2}^{*}\right)$; and (ii) firm 2's behavior is characterized by the first-order conditions (3) and (4) which, evaluated at $\left(q_{A 1}, q_{B 1}\right)=\left(q_{A 1}^{*}, q_{B 1}^{*}\right)$ and $\hat{c}=\hat{c}^{*}$, yield $\left(q_{A 2}, q_{B 2}\right)=\left(0, q_{B 2}^{*}\right)$. We can thus interpret the move from $\left(Q^{\circ}, Q^{\circ}\right)$ to $\left(Q_{A}^{*}, Q_{B}^{*}\right)$ as the evolution of the equilibrium $\left(\hat{Q}_{A}(\hat{c}), \hat{Q}_{B}(\hat{c})\right)$ as $\hat{c}$ increases from $c$ to $\hat{c}^{*}$.

We first consider the effect of single exclusivity on outputs. Differentiating (14) and (15) with respect to $\hat{Q}_{A}, \hat{Q}_{B}$ and $\hat{c}$ yields:

$$
\begin{aligned}
d \hat{Q}_{A}-\partial_{1} \hat{R}\left(\hat{Q}_{B}, \hat{c}\right) d \hat{Q}_{B} & =\partial_{2} \hat{R}\left(\hat{Q}_{B}, \hat{c}\right) d \hat{c} \\
d \hat{Q}_{B} & =R^{\prime}\left(\hat{Q}_{A}\right) d \hat{Q}_{A}
\end{aligned}
$$

and thus:

$$
\begin{aligned}
\hat{Q}_{A}^{\prime}(\hat{c}) & =\frac{\partial_{2} R\left(\hat{Q}_{B}(\hat{c}), \hat{c}\right)}{1-\partial_{1} \hat{R}\left(\hat{Q}_{B}(\hat{c}), \hat{c}\right) R^{\prime}\left(\hat{Q}_{A}(\hat{c})\right)}<0, \\
0 & <\hat{Q}_{B}^{\prime}(\hat{c})=R^{\prime}\left(\hat{Q}_{A}(\hat{c})\right) \hat{Q}_{A}^{\prime}(\hat{c})<-\hat{Q}_{A}^{\prime}(\hat{c}),
\end{aligned}
$$

where the inequalities follow from $-1<R^{\prime}<0,-1<\partial_{1} \hat{R}<0$ and $\partial_{2} \hat{R}<0$. Hence, introducing an exclusive dealing agreement on good $A$ leads to a reduction in the output of good $A$ and, to a lesser extent, to an increase in the output of good $B$ :

$$
Q_{A}^{*}<\frac{Q_{A}^{*}+Q_{B}^{*}}{2}<Q^{\circ}<Q_{B}^{*}
$$

We now turn to the effect of single exclusivity on social welfare. Recall that total welfare is equal to

$$
W\left(Q_{A}, Q_{B}\right)=U\left(Q_{A}, Q_{B}\right)-c Q_{A}-c Q_{B}
$$

where $U\left(Q_{A}, Q_{B}\right)$ represents the gross consumer utility associated with consumption levels $Q_{A}$ and $Q_{B}$, and satisfies, for $i \neq j \in\{A, B\}$ :

$$
\frac{\partial U}{\partial Q_{i}}\left(Q_{A}, Q_{B}\right)=P\left(Q_{i}, Q_{j}\right) .
$$

Hence:

$$
\frac{\partial W}{\partial Q_{i}}\left(Q_{A}, Q_{B}\right)=\frac{\partial U}{\partial Q_{i}}\left(Q_{A}, Q_{B}\right)-c=P\left(Q_{i}, Q_{j}\right)-c .
$$

We now show that

$$
\hat{W}(\hat{c}) \equiv W\left(\hat{Q}_{A}(\hat{c}), \hat{Q}_{B}(\hat{c})\right)
$$


decreases as $\hat{c}$ increases. We have:

$$
\begin{aligned}
\hat{W}^{\prime}(\hat{c}) & =\frac{\partial W}{\partial Q_{A}}\left(\hat{Q}_{A}(\hat{c}), \hat{Q}_{B}(\hat{c})\right) \hat{Q}_{A}^{\prime}(\hat{c})+\frac{\partial W}{\partial Q_{B}}\left(\hat{Q}_{A}(\hat{c}), \hat{Q}_{B}(\hat{c})\right) \hat{Q}_{B}^{\prime}(\hat{c}) \\
& =\left[P\left(\hat{Q}_{A}(\hat{c}), \hat{Q}_{B}(\hat{c})\right)-c\right] \hat{Q}_{A}^{\prime}(\hat{c})+\left[P\left(\hat{Q}_{B}(\hat{c}), \hat{Q}_{A}(\hat{c})\right)-c\right] \hat{Q}_{B}^{\prime}(\hat{c}) \\
& \leq\left[P\left(\hat{Q}_{A}(\hat{c}), \hat{Q}_{B}(\hat{c})\right)-c\right] \hat{Q}_{A}^{\prime}(\hat{c})-\left[P\left(\hat{Q}_{B}(\hat{c}), \hat{Q}_{A}(\hat{c})\right)-c\right] \hat{Q}_{A}^{\prime}(\hat{c}) \\
& =\left[P\left(\hat{Q}_{A}(\hat{c}), \hat{Q}_{B}(\hat{c})\right)-P\left(\hat{Q}_{B}(\hat{c}), \hat{Q}_{A}(\hat{c})\right)\right] \hat{Q}_{A}^{\prime}(\hat{c}),
\end{aligned}
$$

where the inequality uses $\hat{P}_{B}>c$ and $0<\hat{Q}_{B}^{\prime}(\hat{c})<-\hat{Q}_{A}^{\prime}(\hat{c})$. As $\hat{Q}_{A}^{\prime}(\hat{c})<0$, to conclude the argument, it thus suffices to establish that $\hat{P}_{A}<\hat{P}_{B}$; we have:

$$
\begin{aligned}
\frac{d}{d \hat{c}}\left(\hat{P}_{A}-\hat{P}_{B}\right)= & {\left[\partial_{1} P\left(\hat{Q}_{A}(\hat{c}), \hat{Q}_{B}(\hat{c})\right)-\partial_{2} P\left(\hat{Q}_{B}(\hat{c}), \hat{Q}_{A}(\hat{c})\right)\right] \hat{Q}_{A}^{\prime}(\hat{c}) } \\
& -\left[\partial_{1} P\left(\hat{Q}_{B}(\hat{c}), \hat{Q}_{A}(\hat{c})\right)-\partial_{2} P\left(\hat{Q}_{A}(\hat{c}), \hat{Q}_{B}(\hat{c})\right)\right] \hat{Q}_{B}^{\prime}(\hat{c}) \\
> & 0,
\end{aligned}
$$

where the inequality stems from $\hat{Q}_{B}^{\prime}(\hat{c})>0>\hat{Q}_{A}^{\prime}(\hat{c})$ and (A.1) - which, using symmetry, implies $\partial_{1} P\left(Q_{i}, Q_{j}\right)<\partial_{2} P\left(Q_{j}, Q_{i}\right)=\partial_{2} P\left(Q_{i}, Q_{j}\right) \cdot{ }^{44}$ As $\hat{P}_{A}=\hat{P}_{B}=P^{\circ}$ for $\hat{c}=c$, it follows that $\hat{P}_{A}>\hat{P}_{B}$ for any $\hat{c}>c$.

Hence, under Assumption $(B)$, introducing an exclusive dealing agreement decreases welfare. From (P.2), doing so however increases industry profit; it follows that it reduces consumer surplus.

\section{Proof of Proposition 8}

To establish existence, consider a candidate equilibrium in which the two integrated firms do not offer any contracts to each other, i.e., $\tau_{A 2}^{* *}=\varnothing$ and $\tau_{B 1}^{* *}=\varnothing$, and sell $\left(q_{A 1}^{* *}, q_{A 2}^{* *}, q_{B 1}^{* *}, q_{B 2}^{* *}\right)=$ $\left(Q^{* *}, 0,0, Q^{* *}\right)$. To show that there is no profitable deviation, suppose for instance that the integrated $M_{A}-R_{1}$ deviates so as to induce a quantity $\hat{q}_{A 2}$. By assumption, $M_{B}-R_{2}$ does not offer any contract to $M_{A}-R_{1}$ in the candidate equilibrium, and thus we still have $\hat{q}_{B 1}=0$, as in the candidate equilibrium. The resulting quantities, $\tilde{q}_{A 1}\left(\hat{q}_{A 2}, 0\right)$ and $\tilde{q}_{B 2}\left(0, \hat{q}_{A 2}\right)$, are the equilibrium quantities in game $\Gamma$ when $\hat{q}_{B 1}=0$.

We now show that $M_{B}-R_{2}$ can guarantee itself at least the candidate equilibrium profit $\Pi^{* *} / 2 \equiv \Pi\left(Q^{* *}, Q^{* *}\right) / 2$ by simply rejecting $M_{A}-R_{1}$ 's deviant offer. Indeed, by doing so it

\footnotetext{
${ }^{44}$ Demand symmetry implies $\partial_{2} P\left(Q, Q^{\prime}\right)=\partial_{2} P\left(Q^{\prime}, Q\right)\left(=\partial_{12} U\left(Q, Q^{\prime}\right)\right.$, where $U(\cdot, \cdot)$ denotes consumers' gross surplus), and thus $\partial_{12}^{2} P\left(Q, Q^{\prime}\right)=\partial_{22}^{2} P\left(Q^{\prime}, Q\right)$, for all $Q$ and $Q^{\prime}$.
} 
would obtain:

$$
\max _{q_{B 2}} \Pi_{2}\left(0, q_{B 2} ; \tilde{q}_{A 1}\left(\hat{q}_{A 2}, 0\right), 0\right) .
$$

But $(\boldsymbol{\Gamma} .3)$ implies $\tilde{q}_{A 1}\left(\hat{q}_{A 2}, 0\right) \leq \tilde{q}_{A 1}(0,0)=q_{A 1}^{* *}$; as the profit of $M_{B}-R_{2}$ decreases in $q_{A 1}$, the above profit is at least equal to:

$$
\max _{q_{B 2}} \Pi_{2}\left(0, q_{B 2} ; q_{A 1}^{* *}, 0\right)=\frac{\Pi^{* *}}{2} .
$$

Therefore, in order to be profitable, the deviation must increase the aggregate profit:

$$
\tilde{\Pi}\left(\hat{q}_{A 2}, 0\right)>\Pi\left(Q^{* *}, Q^{* *}\right)=\tilde{\Pi}(0,0) .
$$

But this contradicts (P.2).

To establish uniqueness, suppose instead that there exists another equilibrium $\left(\hat{q}_{A 1}, \hat{q}_{A 2}, \hat{q}_{B 1}, \hat{q}_{B 2}\right) \neq$ $\left(Q^{* *}, 0,0, Q^{* *}\right)$. This implies, in particular, that $\hat{q}_{A 2}>0$ or $\hat{q}_{B 1}>0$. Furthermore, by construction we have $\hat{q}_{A 1}=\tilde{q}_{A 1}\left(\hat{q}_{A 2}, \hat{q}_{B 1}\right)$ and $\hat{q}_{B 2}=\tilde{q}_{B 2}\left(\hat{q}_{B 1}, \hat{q}_{A 2}\right)$, and the aggregate equilibrium profit is $\tilde{\Pi}\left(\hat{q}_{A 2}, \hat{q}_{B 1}\right)$. It follows from $(\boldsymbol{\Gamma} .2)$ that this aggregate profit is lower than $\tilde{\Pi}(0,0)=\Pi^{* *}$. Hence, the equilibrium profit of at least one integrated firm, say $M_{A}-R_{1}$, must therefore be strictly less than $\Pi^{* *} / 2$.

Consider first the case where manufacturers are the proposers and suppose that $M_{A}-R_{1}$ deviates by offering no contract to $M_{B}-R_{2}$, implying $q_{A 2}=0$. Given the quantity $q_{B 1} \geq 0$ of good $B$ that $M_{B}-R_{2}$ expects $R_{1}$ to sell in the continuation equilibrium, the other quantities are $\hat{q}_{A 1}^{D}=\tilde{q}_{A 1}\left(0, q_{B 1}\right)$ and $\hat{q}_{B 2}^{D}=\tilde{q}_{B 2}\left(q_{B 1}, 0\right)$. Property $(\boldsymbol{\Gamma} . \mathbf{3})$ implies $\tilde{q}_{B 2}\left(q_{B 1}, 0\right) \leq \tilde{q}_{B 2}(0,0)=Q^{* *}$. As $M_{A}-R_{1}$ 's deviation profit decreases with $q_{B 2}$, it is bounded from below by $\Pi\left(Q^{* *}, Q^{* *}\right) / 2$, a contradiction.

A similar reasoning applies to the case where retailers are the proposers. If $M_{A}-R_{1}$ deviates by offering no contract to $M_{B}-R_{2}$, implying $q_{B 1}=0$. Given the quantity $q_{A 2} \geq 0$ of good $A$ that $M_{B}-R_{2}$ expects $M_{A}$ to pick in the continuation equilibrium, the other quantities are $\hat{q}_{A 1}^{D}=\tilde{q}_{A 1}\left(q_{A 2}, 0\right)$ and $\hat{q}_{B 2}^{D}=\tilde{q}_{B 2}\left(0, q_{A 2}\right) \leq \tilde{q}_{B 2}(0,0)=Q^{* *}$, implying that the deviation profit is at least $\Pi\left(Q^{* *}, Q^{* *}\right) / 2$, a contradiction.

\section{J Proof of Proposition 9}

We first consider the case where manufacturers are the proposers, before turning to the other case. 


\section{J.1 Manufacturers as Proposers}

\section{J.1.1 Candidate equilibrium}

We establish the existence of the following equilibrium:

- $M_{A}$ offers $R_{2}$ a forcing contract, $(\hat{q}, \hat{T})$, whereas $M_{B}$ offers $R_{1}$ a (cost-based) forcing contract, $\left(q_{B 1}^{*}, T_{B 1}^{*}\right)$, and offers $R_{2}$ to supply any quantity at cost (i.e., $\tau_{B 2}(q)=c q$, for any $q \geq 0)$.

- $R_{2}$ is indifferent between accepting both $M_{A}$ 's and $M_{B}$ 's contracts, or either one of them, and rejects $M_{A}$ 's offer;

- $R_{1}$ is indifferent between accepting and rejecting $M_{B}$ 's contract, and accepts it.

By construction, the equilibrium quantities are the same as in the asymmetric Cournot duopoly introduced in Section 3, and the equilibrium profits are equal to:

$$
\begin{aligned}
\pi_{M_{A}-R_{1}}^{*} & =\left[P\left(q_{A 1}^{*}, q_{B 1}^{*}+q_{B 2}^{*}\right)-c\right] q_{A 1}^{*}+P\left(q_{B 1}^{*}+q_{B 2}^{*}, q_{A 1}^{*}\right) q_{B 1}^{*}-T_{B 1}^{*}, \\
\pi_{R_{2}}^{*} & =\left[P\left(q_{B 1}^{*}+q_{B 2}^{*}, q_{A 1}^{*}\right)-c\right] q_{B 2}^{*}, \\
\pi_{M_{B}}^{*} & =T_{B 1}^{*}-c q_{B 1}^{*} .
\end{aligned}
$$

Determination of $T_{B 1}^{*} \quad$ In equilibrium, $R_{1}$ must be indifferent between rejecting or accepting $M_{B}$ 's offer:

- $R_{1}$ should not benefit from rejecting the offer, otherwise it would do so;

- conversely, if $R_{1}$ were strictly better off accepting the offer, then $M_{B}$ could slightly increase its fee: as $M_{B}$ is independent, this would not affect $R_{1}$ 's beliefs about $M_{A}$ 's behavior (that is, $R_{1}$ 's wary beliefs boil down to passive beliefs), and thus $R_{1}$ would accept $M_{B}$ 's deviant offer, making the deviation profitable.

If $R_{1}$ rejects $M_{B}$ 's offer, it then sells $\tilde{q}_{A 1}$ units of good $A$, where:

$$
\tilde{q}_{A 1} \equiv \arg \max _{q_{A 1}}\left[P\left(q_{A 1}, q_{B 2}^{*}\right)-c\right] q_{A 1}
$$

and thus obtains a profit equal to:

$$
\tilde{\pi}_{M_{A}-R_{1}} \equiv\left[P\left(\tilde{q}_{A 1}, q_{B 2}^{*}\right)-c\right] \tilde{q}_{A 1}
$$


Therefore, the fee $T_{B 1}^{*}$ is such that $\pi_{M_{A}-R_{1}}^{*}=\tilde{\pi}_{M_{A}-R_{1}}$, or:

$$
T_{B 1}^{*}=\left[P\left(q_{A 1}^{*}, q_{B 1}^{*}+q_{B 2}^{*}\right)-c\right] q_{A 1}^{*}+P\left(q_{B 1}^{*}+q_{B 2}^{*}, q_{A 1}^{*}\right) q_{B 1}^{*}-\left[P\left(\tilde{q}_{A 1}, q_{B 2}^{*}\right)-c\right] \tilde{q}_{A 1} .
$$

This in particular ensures that $M_{B}$ 's equilibrium profit is non-negative:

$$
\begin{aligned}
\pi_{M_{B}}^{*}= & T_{B 1}^{*}-c q_{B 1}^{*} \\
= & {\left[P\left(q_{A 1}^{*}, q_{B 1}^{*}+q_{B 2}^{*}\right)-c\right] q_{A 1}^{*}+\left[P\left(q_{B 1}^{*}+q_{B 2}^{*}, q_{A 1}^{*}\right)-c\right] q_{B 1}^{*}-\left[P\left(\tilde{q}_{A 1}, q_{B 2}^{*}\right)-c\right] \tilde{q}_{A 1} } \\
= & \max _{q_{A 1}, q_{B 1}}\left\{\left[P\left(q_{A 1}, q_{B 1}+q_{B 2}^{*}\right)-c\right] q_{A 1}+\left[P\left(q_{B 1}+q_{B 2}^{*}, q_{A 1}\right)-c\right] q_{B 1}\right\} \\
& -\max _{q_{A 1}}\left[P\left(q_{A 1}, q_{B 1}+q_{B 2}^{*}\right)-c\right] q_{A 1} \\
\geq & 0 .
\end{aligned}
$$

Determination of $(\hat{q}, \hat{T})$ The described equilibrium is such that $R_{2}$ can also obtain its equilibrium profit $\pi_{R_{2}}^{*}$ by accepting only $M_{A}$ 's contract $(\hat{q}, \hat{T})$, and by dealing with both manufacturers. As $M_{B}$ offers $R_{2}$ to supply any quantity at cost, this in turn requires $\hat{q}$ to be "large enough" so as to ensure that, conditional on selling $\hat{q}$ units of good $A, R_{2}$ does not want to sell good B. Assumptions (A.1) and (A.2) ensure that such large values exist for $\hat{q}$.

As $R_{2}$ would obtain $P\left(q_{A 1}^{*}+\hat{q}, q_{B 1}^{*}\right) \hat{q}-\hat{T}$ by selecting $M_{A}$ 's offer $(\hat{q}, \hat{T})$ instead of $M_{B}$ 's offer, we must have:

$$
\hat{T}=P\left(q_{A 1}^{*}+\hat{q}, q_{B 1}^{*}\right) \hat{q}-\left[P\left(q_{B 1}^{*}+q_{B 2}^{*}, q_{A 1}^{*}\right)-c\right] q_{B 2}^{*} .
$$

\section{J.1.2 Deviations}

The above characterization of equilibrium quantities and fees ensures that retailers have no profitable deviation, neither at the acceptance stage nor at the product market competition stage. We thus now focus on manufacturers' deviations at the offer stage.

Deviations by $M_{B}$ Given the passive beliefs assumption, it suffices to consider one-sided deviations. Also, by construction such a one-sided deviation cannot be profitable if it is not accepted, as in equilibrium $M_{B}$ makes a non-negative profit with both $R_{1}$ and $R_{2}$.

Consider first a deviant offer to $R_{1}$. Such a deviation cannot reduce $M_{A}-R_{1}$ 's payoff, which it can secure by rejecting $M_{B}$ 's offer. But it cannot increase the joint profit that $M_{B}$ generates with $M_{A}-R_{1}$ through $R_{1}$ 's sales either, as the equilibrium contract offered to $R_{1}$ is bilaterally efficient. 
Consider now a deviant offer to $R_{2}$. Again, such a deviation cannot reduce $R_{2}$ 's payoff, which it can secure by rejecting $M_{B}$ 's offer and accepting instead $M_{A}$ 's offer. And it cannot increase the joint profit that $M_{B}$ generates with $R_{2}$ either, as the equilibrium contract offered to $R_{2}$ is bilaterally efficient, regardless of whether $R_{2}$ accepts or rejects $M_{A}$ 's offer.

Deviations by $M_{A}-R_{1}$ • Consider first a deviant offer by $M_{A}-R_{1}$ that induces $R_{2}$ to reject it.

Suppose first that, in the continuation equilibrium, $R_{2}$ accepts $M_{B}$ 's offer. If $R_{1}$ also accepts $M_{B}$ 's offer, then the continuation equilibrium quantities are $\left(q_{A 1}^{*}, q_{B 1}^{*}, q_{B 2}^{*}\right) ; M_{A}-R_{1}$ thus obtains its equilibrium profit, making the deviation unprofitable. If instead $R_{1}$ rejects $M_{B}$ 's offer in the continuation equilibrium then, from $(\boldsymbol{\Gamma} .3), R_{2}$ puts on the market a larger quantity $q_{B 2}=$ $\tilde{q}_{B 2}(0,0)>q_{B 2}^{*}=\tilde{q}_{B 2}\left(q_{B 1}^{*}, 0\right)$, implying that $M_{A}-R_{1}$ obtains less than its equilibrium profit, making the deviation unprofitable.

Therefore, to be profitable, the deviation must induce $R_{2}$ to reject $M_{B}$ 's offer. We can distinguish two cases, depending on $R_{1}$ 's acceptance decision of $M_{B}$ 's offer:

- If $R_{1}$, too, rejects $M_{B}$ 's offer in the continuation equilibrium, then it chooses to sell $q_{A 1}$ units of $\operatorname{good} A$ so as to maximize

$$
\left[P\left(q_{A 1}, 0\right)-c\right] q_{A 1}
$$

This ensures that the resulting price of good $A$ satisfies $p_{A}=P\left(q_{A 1}, 0\right)>c$. But then, $p_{B}=P\left(0, q_{A 1}\right)>P\left(q_{A 1}, 0\right)>c$ by Assumption (A.1), which in turn implies that $R_{2}$ would rather accept $M_{B}$ 's offer and sell a positive quantity of good $B$, in contradiction with $R_{2}$ 's supposed rejection of $M_{B}$ 's offer.

- If instead $R_{1}$ accepts $M_{B}$ 's offer in the continuation equilibrium, then it sells $q_{B 1}^{*}$ units of $\operatorname{good} B$ and $q_{A 1}^{a}$ units of good $A$ so as to maximize

$$
\left[P\left(q_{A 1}, q_{B 1}^{*}\right)-c\right] q_{A 1}+P\left(q_{B 1}^{*}, q_{A 1}\right) q_{B 1}^{*}-T_{B 1}^{*}
$$

By revealed preference, this must exceed the profit it could achieve by rejecting $M_{B}$ 's offer and selling only good $A$, which implies:

$$
\begin{aligned}
P\left(q_{B 1}^{*}, q_{A 1}^{a}\right) q_{B 1}^{*} & \geq T_{B 1}^{*}+\max _{q_{A 1}}\left[P\left(q_{A 1}, 0\right)-c\right] q_{A 1}-\left[P\left(q_{A 1}^{a}, q_{B 1}^{*}\right)-c\right] q_{A 1}^{a} \\
& >T_{B 1}^{*}+\max _{q_{A 1}}\left[P\left(q_{A 1}, 0\right)-c\right] q_{A 1}-\left[P\left(q_{A 1}^{a}, 0\right)-c\right] q_{A 1}^{a} \\
& \geq c q_{B 1}^{*}
\end{aligned}
$$


where the strict inequality follows from Assumption (A.1) and the last inequality stems from (16). Therefore, $P\left(q_{B 1}^{*}, q_{A 1}^{a}\right)>c$, which again implies that $R_{2}$ would rather accept $M_{B}$ 's offer and sell a positive quantity of $\operatorname{good} B$, in contradiction with $R_{2}$ 's supposed rejection of $M_{B}$ 's offer.

- Consider now a deviant offer by $M_{A}-R_{1}$ that is accepted by $R_{2}$ together with $M_{B}$ 's offer.

Let $\hat{q}_{A 2}$ denote the quantity of good $A$ sold by $R_{2}$, and $\hat{T}_{A 2}$ the associated payment to $M_{A}$, in the continuation equilibrium.

Suppose first that, in the continuation equilibrium, $R_{1}$ also keeps accepting $M_{B}$ 's offer. By construction, $M_{B}$ still obtains its equilibrium profit, $\pi_{M_{B}}^{*}=T_{B 1}^{*}-c q_{B 1}^{*}$. Furthermore, by deviating from this continuation equilibrium and rejecting the offer, $R_{2}$ can ensure itself a profit equal to:

$$
\max _{q_{B 2}}\left[P\left(q_{B 1}^{*}+q_{B 2}, \tilde{q}_{A 1}\left(\hat{q}_{A 2}, q_{B 1}^{*}\right)\right)-c\right] q_{B 2} .
$$

Therefore, $M_{A}-R_{1}$ 's deviation profit cannot exceed

$$
\varphi\left(\hat{q}_{A 2}\right) \equiv \tilde{\Pi}\left(\hat{q}_{A 2}, q_{B 1}^{*}\right)-\pi_{M_{B}}^{*}-\max _{q_{B 2}}\left[P\left(q_{B 1}^{*}+q_{B 2}, \tilde{q}_{A 1}\left(\hat{q}_{A 2}, q_{B 1}^{*}\right)\right)-c\right] q_{B 2} .
$$

From (Г.4), we have:

$$
\begin{aligned}
\varphi\left(\hat{q}_{A 2}\right) & \leq \varphi(0) \\
& =\tilde{\Pi}\left(0, q_{B 1}^{*}\right)-\pi_{M_{B}}^{*}-\max _{q_{B 2}}\left[P\left(q_{B 1}^{*}+q_{B 2}, \tilde{q}_{A 1}\left(0, q_{B 1}^{*}\right)\right)-c\right] q_{B 2} \\
& =\tilde{\Pi}\left(0, q_{B 1}^{*}\right)-\pi_{M_{B}}^{*}-\max _{q_{B 2}}\left[P\left(q_{B 1}^{*}+q_{B 2}, q_{A 1}^{*}\right)-c\right] q_{B 2} \\
& =\tilde{\Pi}\left(0, q_{B 1}^{*}\right)-\pi_{M_{B}}^{*}-\left[P\left(q_{B 1}^{*}+q_{B 2}^{*}, q_{A 1}^{*}\right)-c\right] q_{B 2}^{*} \\
& =\tilde{\Pi}\left(0, q_{B 1}^{*}\right)-\pi_{M_{B}}^{*}-\pi_{R_{2}}^{*} \\
& =\pi_{M_{A}-R_{1}}^{*}
\end{aligned}
$$

where the second equality stems from $\tilde{q}_{A 1}\left(0, q_{B 1}^{*}\right)=q_{A 1}^{*}$ and the third one stems from $\tilde{q}_{B 2}\left(q_{B 1}^{*}, 0\right)=$ $q_{B 2}^{*}$. It follows that the deviation is not profitable for $M_{A}-R_{1}$.

Remark 1 Applying this reasoning to a deviant offer equal to the equilibrium shadow offer $(\hat{q}, \hat{T})$ shows that, in equilibrium, $M_{A}-R_{1}$ strictly prefers that $R_{2}$ rejects $M_{A}$ 's offer.

Remark 2 The previous remark does not necessarily imply that offering $(\hat{q}, \hat{T})$ is a dominated strategy for $M_{A}-R_{1}$ : this will indeed not be the case if there exists a $q_{B 1}$ (together with an appropriate best response $q_{A 1}$ ) and a $q_{B 2}$ such that $M_{A}-R_{1}$ is better off when $R_{2}$ mistakenly accepts $(\hat{q}, \hat{T})$. 
Suppose now that, in the continuation equilibrium, $R_{1}$ rejects $M_{B}$ 's offer. The quantities are then the same as in the equilibrium of game $\Gamma$ for $\left(\hat{q}_{A 2}\right.$ and $) \hat{q}_{B 1}=0$. We first note that this continuation equilibrium (referred to below with superscript $c$ ) is less profitable for $M_{A}-R_{1}$ than the "alternative equilibrium" (referred to below with a superscript $a$ ) in which the quantities are those of the equilibrium of game $\Gamma$ for $\hat{q}_{A 2}=\hat{q}_{B 1}=0$ (and $M_{B}$ supplies $R_{2}$ at cost). To see this, note that :

- $M_{B}$ makes the same profit in both equilibrium scenarios: $\pi_{M_{B}}^{c}=\pi_{M_{B}}^{a}=0$.

- $R_{2}$ does not make more profit in the alternative equilibrium than in the continuation equilibrium, i.e.: $\pi_{R_{2}}^{c} \geq \pi_{R_{2}}^{a}$. To show this, note first that the continuation equilibrium must give $R_{2}$ at least the profit that it could secure by deviating and only accepting $M_{B}$ 's offer:

$$
\pi_{R_{2}}^{c} \geq \max _{q_{B 2}}\left[P\left(q_{B 2}, q_{A 1}^{c}\right)-c\right] q_{B 2} .
$$

But the right-hand side (weakly) exceeds

$$
\pi_{R_{2}}^{a}=\max _{q_{B 2}}\left[P\left(q_{B 2}, q_{A 1}^{a}\right)-c\right] q_{B 2},
$$

as $q_{A 1}^{a}=\tilde{q}_{A 1}(0,0) \geq q_{A 1}^{c}=\tilde{q}_{A 1}\left(0, \hat{q}_{A 2}\right)$, from Property $(\boldsymbol{\Gamma} .3)$. Hence, $\pi_{R_{2}}^{c} \geq \pi_{R_{2}}^{a}$.

- Property $(\boldsymbol{\Gamma} .2)$ ensures that the aggregate profit is larger in the alternative equilibrium than in the continuation equilibrium:

$$
\pi_{M_{B}}^{a}+\pi_{M_{A}-R_{1}}^{a}+\pi_{R_{2}}^{a} \geq \pi_{M_{B}}^{c}+\pi_{M_{A}-R_{1}}^{c}+\pi_{R_{2}}^{c}
$$

It follows that the integrated firm makes more profit in the alternative equilibrium than in the continuation equilibrium:

$$
\pi_{M_{A}-R_{1}}^{a} \geq \pi_{M_{A}-R_{1}}^{c}
$$

But $\pi_{M_{A}-R_{1}}^{a}<\tilde{\pi}_{M_{A}-R_{1}}=\max _{q_{A 1}}\left[P\left(q_{A 1}, q_{B 2}^{*}\right)-c\right] q_{A 1}$, as $R_{2}$ is more aggressive in the alternative equilibrium than in the "pseudo duopoly" scenario in which $R_{1}$ carries $A$ only and $R_{2}$ carries $B$ only, but $R_{2}$ anticipates that $R_{1}$ is also carrying $B$, and thus sells $q_{B 2}^{*}=\tilde{q}_{B 2}\left(q_{B 1}^{*}, 0\right)$ rather than $q_{B 2}=\tilde{q}_{B 2}(0,0)$. As $\tilde{\pi}_{M_{A}-R_{1}}=\pi_{M_{A}-R_{1}}^{*}$, we have:

$$
\pi_{M_{A}-R_{1}}^{c} \leq \pi_{M_{A}-R_{1}}^{a} \leq \pi_{M_{A}-R_{1}}^{*}
$$

That is, $M_{A}-R_{1}$ 's deviation is not profitable.

- Finally, consider now a deviation by $M_{A}-R_{1}$ that induces $R_{2}$ to drop $M_{B}$ 's offer. 
As $R_{2}$ can costlessly accept $M_{B}$ 's offer to supply at cost, and then choose $q_{B 2}=0$, the above reasoning (for deviations inducing $R_{2}$ to accept the deviant offer by $M_{A}$ as well as $M_{B}$ 's offer) still applies, which concludes the proof.

\section{J.2 Retailers as Proposers}

\section{J.2.1 Existence}

We establish the existence of the following equilibrium:

- $R_{1}$ offers $M_{B}$ a (cost-based) forcing contract, $\left(q_{B 1}^{*}, c q_{B 1}^{*}\right)$, which $M_{B}$ accepts.

- $R_{2}$ offers no contract to $M_{A}$ and offers $M_{B}$ a (cost-based) forcing contract, $\left(q_{B 2}^{*}, c q_{B 2}^{*}\right)$, which $M_{B}$ accepts.

As before, the equilibrium quantities are the same as in the asymmetric Cournot duopoly introduced in Section 3. The equilibrium profits are equal to:

$$
\begin{aligned}
\pi_{M_{A}-R_{1}}^{*} & =\left[P\left(q_{A 1}^{*}, q_{B 1}^{*}+q_{B 2}^{*}\right)-c\right] q_{A 1}^{*}+\left[P\left(q_{B 1}^{*}+q_{B 2}^{*}, q_{A 1}^{*}\right)-c\right] q_{B 1}^{*}, \\
\pi_{R_{2}}^{*} & =\left[P\left(q_{B 1}^{*}+q_{B 2}^{*}, q_{A 1}^{*}\right)-c\right] q_{B 2}^{*}, \\
\pi_{M_{B}}^{*} & =0 .
\end{aligned}
$$

Clearly, $M_{B}$ cannot benefit from rejecting any of the offers. Likewise, the integrated firm cannot profitably deviate by offering a different contract (or no contract) to $M_{B}$, and $R_{2}$ cannot profitably deviate by simply offering a different contract (or no contract) to $M_{B}$ without also making an offer to $M_{A}-R_{1}$. Hence, we have to check only for deviations where $R_{2}$ offers a contract to $M_{A}-R_{1}$.

Without loss of generality, we can restrict attention to deviations in which $R_{2}$ offers a forcing contract $\left(\hat{q}_{A 2}, \hat{T}_{A 2}\right)$ to $M_{A}-R_{1}$ and a cost-based forcing contract $\left(\tilde{q}_{B 2}\left(\hat{q}_{A 2}, q_{B 1}^{*}\right), c \tilde{q}_{B 2}\left(\hat{q}_{A 2}, q_{B 1}^{*}\right)\right)$ to $M_{B}$. Note that, in the continuation equilibrium, $M_{B}$ keeps accepting $M_{A}-R_{1}$ 's equilibrium offer, $\left(q_{B 1}^{*}, c q_{B 1}^{*}\right)$.

- Having wary beliefs, the integrated $M_{A}-R_{1}$ is willing to accept this deviant offer if and only if

$$
\begin{aligned}
& {\left[\hat{T}_{A 2}-c \hat{q}_{A 2}\right]+\tilde{\Pi}_{1}\left(\hat{q}_{A 2}, q_{B 1}^{*}\right) } \\
\geq & \left.\max _{q_{A 1}}\left\{\left[P\left(q_{A 1}, q_{B 1}^{*}+\tilde{q}_{B 2}\left(\hat{q}_{A 2}, q_{B 1}^{*}\right)\right)-c\right] q_{A 1}+\left[P\left(q_{B 1}^{*}+\tilde{q}_{B 2}\left(\hat{q}_{A 2}, q_{B 1}^{*}\right), q_{A 1}\right)-c\right] q_{B 1}^{*}\right\}\right)
\end{aligned}
$$


where the right-hand side is the maximum that $M_{A}-R_{1}$ can get by rejecting the offer (thereby taking $R_{2}$ by surprise). The optimal deviation by $R_{2}$ is such that this inequality holds with equality (as, otherwise, $R_{2}$ would do better by slightly lowering $\hat{T}_{A 2}$ ).

- This deviation is profitable for $R_{2}$ if and only if

$$
\tilde{\Pi}_{2}\left(\hat{q}_{A 2}, q_{B 1}^{*}\right)-\left[\hat{T}_{A 2}-c \hat{q}_{A 2}\right]>\tilde{\Pi}_{2}\left(0, q_{B 1}^{*}\right)
$$

Adding-up (17) and (18) yields

$$
\begin{aligned}
& \tilde{\Pi}\left(\hat{q}_{A 2}, q_{B 1}^{*}\right)-\max _{q_{A 1}}\left\{\left[P\left(q_{A 1}, q_{B 1}^{*}+\tilde{q}_{B 2}\left(q_{B 1}^{*}, \hat{q}_{A 2}\right)\right)-c\right] q_{A 1}+\left[P\left(q_{B 1}^{*}+\tilde{q}_{B 2}\left(q_{B 1}^{*}, \hat{q}_{A 2}\right), q_{A 1}\right)-c\right] q_{B 1}^{*}\right\} \\
> & \tilde{\Pi}_{2}\left(0, q_{B 1}^{*}\right) .
\end{aligned}
$$

But by $(\boldsymbol{\Gamma} .4)$, the left-hand side is maximized at $\hat{q}_{A 2}=0$, where it is equal to the right-hand side; hence, the deviation cannot be profitable.

\section{J.2.2 Uniqueness}

We now show that, under a mild regularity condition, there does not exist an equilibrium with cross-selling between $M_{A}$ and $R_{2}$. To see this, consider a candidate equilibrium $\left(\tau_{i j}^{e}\right)_{i=A, B, j=1,2}$ inducing quantities $\left(q_{i j}^{e}\right)_{i=A, B, j=1,2}$ such that $q_{A 2}^{e}>0$. In equilibrium, (i) $M_{B}$ must supply $q_{B 1}^{e}$ at cost (as $M_{B}$ is willing to supply any quantity at cost, it is optimal for $M_{A}-R_{1}$ to set $\left.\tau_{B 1}^{e}\left(q_{B 1}^{e}\right)=c q_{B 1}^{e}\right)$, and (ii) $M_{A}$ must be indifferent between accepting $R_{2}$ 's contract offer and not (if $M_{A}$ were to prefer strictly accepting the offer, $R_{2}$ would have an incentive to worsen the terms). Hence, the integrated $M_{A}-R_{1}$ 's equilibrium profit must satisfy

$$
\pi_{M_{A}-R_{1}}^{e}=\max _{q_{A 1}}\left\{\left[P\left(q_{A 1}, q_{B 1}^{e}+q_{B 2}^{e}\right)-c\right] q_{A 1}+\left[P\left(q_{B 1}^{e}+q_{B 2}^{e}, q_{A 1}\right)-c\right] q_{B 1}^{e}\right\} .
$$

At the same time, $M_{A}-R_{1}$ must not have an incentive to deviate by (i) changing its contract offer to $M_{B}$ at the first stage and then (ii) rejecting $R_{2}$ 's contract offer and adjusting its own downstream output $q_{A 1}$ at the second stage. As $M_{B}$ is willing to supply any quantity $q_{B 1}$ at cost, we must have

$$
\pi_{M_{A}-R_{1}}^{e} \geq \max _{q_{A 1}, q_{B 1}}\left\{\left[P\left(q_{A 1}, q_{B 1}+q_{B 2}^{e}\right)-c\right] q_{A 1}+\left[P\left(q_{B 1}+q_{B 2}^{e}, q_{A 1}\right)-c\right] q_{B 1}\right\} .
$$

Combining these two conditions yields:

$$
\begin{gathered}
\max _{q_{A 1}}\left\{\left[P\left(q_{A 1}, q_{B 1}^{e}+q_{B 2}^{e}\right)-c\right] q_{A 1}+\left[P\left(q_{B 1}^{e}+q_{B 2}^{e}, q_{A 1}\right)-c\right] q_{B 1}^{e}\right\} \\
\geq \max _{q_{A 1}, q_{B 1}}\left\{\left[P\left(q_{A 1}, q_{B 1}+q_{B 2}^{e}\right)-c\right] q_{A 1}+\left[P\left(q_{B 1}+q_{B 2}^{e}, q_{A 1}\right)-c\right] q_{B 1}\right\} .
\end{gathered}
$$


However, $M_{A}-R_{1}$ equilibrium quantities must also satisfy

$$
\left(q_{A 1}^{e}, q_{B 1}^{e}\right) \in \max _{q_{A 1}, q_{B 1}}\left\{\left[P\left(q_{A 1}+q_{A 2}^{e}, q_{B 1}+q_{B 2}^{e}\right)-c\right] q_{A 1}+\left[P\left(q_{B 1}+q_{B 2}^{e}, q_{A 1}+q_{A 2}^{e}\right)-c\right] q_{B 1}\right\}
$$

We thus must have:

$$
\max _{q_{B 1}} \varphi\left(q_{B 1} ; 0\right)=\max _{q_{B 1}} \varphi\left(q_{B 1} ; q_{A 2}^{e}\right)
$$

where

$$
\varphi\left(q_{B 1} ; q_{A 2}\right) \equiv \max _{q_{A 1}}\left\{\left[P\left(q_{A 1}+q_{A 2}^{e}, q_{B 1}+q_{B 2}^{e}\right)-c\right] q_{A 1}+\left[P\left(q_{B 1}+q_{B 2}^{e}, q_{A 1}+q_{A 2}^{e}\right)-c\right] q_{B 1}\right\} .
$$

If follows that $q_{A 2}^{e}$ must be zero if $\arg \max _{q_{B 1}} \varphi\left(q_{B 1} ; q_{A 2}\right)$ varies with $q_{A 2}$. 


\section{References}

[1] Allain, M.-L., and C. Chambolle (2013). "Anti-Competitive effects of Resale-Below-Cost Laws," International Journal of Industrial Organization, 29(4): 373-385.

[2] Bernheim, B. D., and M. D. Whinston (1985). "Common Marketing Agency as a Device for Facilitating Collusion," RAND Journal of Economics, 16(2): 269-281.

[3] Bernheim, B. D., and M. D. Whinston (1986a). "Menu Auctions, Resource Allocation, and Economic Influence," Quarterly Journal of Economics, 101(1): 1-32.

[4] Bernheim, B. D., and M. D. Whinston (1986b). "Common Agency," Econometrica, 54(4): 923-942.

[5] Bernheim, B. D., and M. D. Whinston (1998). "Exclusive Dealing," Journal of Political Economy, 106(1):64-103.

[6] Bonanno, G., and J. Vickers (1988). "Vertical Separation," Journal of Industrial Economics, 36(3): 257-265.

[7] Caillaud, B., and P. Rey (1995). "Strategic Aspects of Delegation," European Economic Review, 39(3-4): 421-431.

[8] Chemla. G. (2003). "Downstream Competition, Foreclosure, and Vertical Integration," Journal of Economics \&3 Management Strategy, 12(2): 261-289.

[9] Chipty, T., and C. M. Snyder (1999). "The role of firm size in bilateral bargaining: A study of the cable television industry," Review of Economics and Statistics, 81(2): 326-340.

[10] Collard-Wexler, A., G. Gowrisankaran and R. S. Lee (2015). "Nash-in-Nash" Bargaining: A Microfoundation for Applied Work," mimeo, available at http://www.people.fas.harvard.edu/ robinlee/papers/BargainingInBilateralOligopoly.pdf.

[11] Crawford, G., and A. Yurukoglu (2012). "The welfare effects of bundling in multichannel television markets," American Economic Review, 102(2): 643-685.

[12] Crawford, G. S., R. S. Lee, M. D. Whinston, and A. Yurukoglu (2015). "The Welfare Effects of Vertical Integration in Multichannel Television Markets," NBER Working Paper No. 21832. 
[13] Crémer, J., and M. Riordan (1987). "On Governing Multilateral Transactions with Bilateral Contracts," RAND Journal of Economics, 18(2): 436-451.

[14] de Fontenay, Catherine, and Joshua Gans (2005), "Vertical Integration in the Presence of Upstream Competition," RAND Journal of Economics, 35(3): 544-572.

[15] de Fontenay, Catherine, and Joshua Gans (2014), "Bilateral Bargaining with Externalities," Journal of Industrial Economics, 62(4): 756-788.

[16] Dobson, P. W., and M. Waterson (2007). "The Competition Effects of Industry-Wide Vertical Price Fixing in Bilateral Oligopoly," International Journal of Industrial Organization, 25(5): 935-962.

[17] de Fontenay, C. C., and J. S. Gans (2005). "Vertical Integration in the Presence of Upstream Competition," RAND Journal of Economics, 36(3): 544-572.

[18] Gal-Or, E. (1991). "Duopolistic Vertical Restraints," European Economic Review, 35(6): 1237-1253.

[19] Gowrisankaran, G., A. Nevo, and R. Town (2015). "Mergers When Prices Are Negotiated: Evidence from the Hospital Industry," American Economic Review, 105(1): 172-203.

[20] Ho, K., and R. S. Lee (2015). "Insurer Competition in Health Care Markets," CEPR Discussion Paper No. DP10812.

[21] Hart, O., and J. Tirole (1990). "Vertical Integration and Market Foreclosure," Brookings Papers on Economic Activity. Microeconomics, 1990, 205-276.

[22] Horn, H. , and A. Wolinsky (1988). "Bilateral Monopolies and Incentives for Mergers," RAND Journal of Economics, 19(3): 408-419.

[23] Jullien, B., and P. Rey (2007). "Resale Price Maintenance and Collusion," RAND Journal of Economics, 38(4): 983-1001.

[24] Lee, R .S., and K. Fong (2013). "Markov-Perfect Network Formation: An Applied Framework for Bilateral Oligopoly and Bargaining in Buyer-Seller Networks," mimeo, available at http://www.people.fas.harvard.edu/ robinlee/papers/MPNENetworkFormation.pdf.

[25] McAfee, R. P., and M. Schwartz (1994). "Opportunism in Multilateral Vertical Contracting: Nondiscrimination, Exclusivity, and Uniformity," American Economic Review, 84(1): 210230. 
[26] Miklós-Thal, J., P. Rey and T. Vergé (2011). "Buyer Power and Intrabrand Coordination," Journal of the European Economic Association, 9(4): 721-741.

[27] Nocke, V., and L. White (2007). "Do Vertical Mergers Facilitate Upstream Collusion?" American Economic Review, 97(4):1321-1339.

[28] Nocke, V., and L. White (2010). "Vertical Merger, Collusion, and Disruptive Buyers," International Journal of Industrial Organization, 28(4):350-354.

[29] O'Brien, D. P., and G. Shaffer (1992). "Vertical Control with Bilateral Contracts," RAND Journal of Economics, 23(3): 299-308.

[30] O’Brien, D. P., and G. Shaffer (1997). "Nonlinear Supply Contracts, Exclusive Dealing, and Equilibrium Market Foreclosure," Journal of Economics $\&$ Management Strategy, 6(4): $755-785$.

[31] Ordover, J., G. Saloner and S. C. Salop (1990). "Equilibrium Market Foreclosure," American Economic Review, 80(1): 127-142.

[32] Piccolo, S., and J. Miklós-Thal (2012). "Colluding through Suppliers," RAND Journal of Economics, 43(3): 492-513.

[33] Reiffen, D. (1992). "Equilibrium Vertical Foreclosure: Comment," American Economic Review, 82(3): 694-697.

[34] Rey, P., and J. Stiglitz (1988). "Vertical Restraints and Producers' Competition," European Economic Review, 32(2-3): 561-568.

[35] Rey, P., and J. Stiglitz (1995). "The Role of Exclusive Territories in Producers' Competition," RAND Journal of Economics, 26(3): 431-451.

[36] Rey, P., and J. Tirole (2007). "A Primer on Foreclosure," Handbook of Industrial Organization Vol. III, M. Armstrong and R. Porter eds, North Holland.

[37] Rey, P., and T. Vergé (2004). "Bilateral Control with Vertical Contracts," RAND Journal of Economics, 35(4): 728-746.

[38] Rey, P., and T. Vergé (2010). "Resale Price Maintenance and Interlocking Relationships," Journal of Industrial Economics, 58(4): 928-961. 
[39] Rey, P., and M. D. Whinston (2013). "Does Retailer Power Lead to Exclusion?," RAND Journal of Economics, 44(1): 75-81.

[40] Salinger, M. A. (1988). "Vertical Mergers and Market Foreclosure," Quarterly Journal of Economics, 77(2): 345-356.

[41] Smith, H., and J. Thanassoulis (2015). "Prices, Profits, and Pass-through of Costs along a Supermarket Supply Chain: Bargaining and Competition," Oxford Review of Economic Policy, 31(1): 64-89.

[42] Whinston, M. D. (2006). Lectures on Antitrust Economics, MIT Press. 


\section{Online Appendix Not for publication}

In this online Appendix, we provide regularity conditions on demand ensuring that the various games considered in the paper have the desired properties. Section A studies the Cournot duopoly games introduced in Section 3 of the paper and provides general conditions on demand ensuring that these duopoly games satisfy Property (P.1). Section B provides an additional condition on demand ensuring that these duopoly games also satisfy Property (P.2). Section C turns to the hypothetical duopoly game introduced in Section 5.3 of the paper, and provides a condition ensuring that this game satisfies Property (B). Finally, Section D considers the game $\Gamma$ introduced in Section 6.1 and provides general conditions on demand ensuring that this game satisfies Properties $(\boldsymbol{\Gamma} .1)-(\boldsymbol{\Gamma} .3)$.

\section{A Cournot Duopoly}

We consider three Cournot duopoly settings with differentiated goods. In all variants there are two goods, $A$ and $B$, and two firms, 1 and 2 :

- The goods are symmetrically differentiated and the inverse demand for good $i=A, B$ is given by $P\left(Q_{i}, Q_{j}\right), j \neq i \in\{A, B\}$. $^{1}$

- The two firms are perfectly substitutable, except possibly for their product portfolios.

The two goods are produced at the same constant unit cost $c$, but the different settings vary in which firms can produce these goods. Specifically, in the first variant (studied in Section A.1 of the paper), one firm can only produce good $A$, and the other firm can only produce good $B$. In the second variant (studied in Section A.2), both firms can produce both goods. Finally, in the third variant (studied in Section A.3), firm 1 can still produce both goods, but firm 2 can only produce good $A$.

Throughout the analysis, we maintain the two assumptions already introduced in the text:

(A.1) For any $\left(Q_{A}, Q_{B}\right) \geq 0,{ }^{2}$

$$
\partial_{1} P\left(Q_{A}, Q_{B}\right) \leq \partial_{2} P\left(Q_{A}, Q_{B}\right) \leq 0,
$$

\footnotetext{
${ }^{1}$ Demand symmetry implies $\partial_{2} P\left(Q, Q^{\prime}\right)=\partial_{2} P\left(Q^{\prime}, Q\right)\left(=\partial_{12} U\left(Q, Q^{\prime}\right)\right.$, where $U(\cdot, \cdot)$ denotes consumers' gross surplus), and thus $\partial_{12}^{2} P\left(Q, Q^{\prime}\right)=\partial_{22}^{2} P\left(Q^{\prime}, Q\right)$, for all $Q$ and $Q^{\prime}$.

${ }^{2}$ Throughout the paper, $\partial_{n} f$ denotes the partial derivative of the function $f$ with respect to its $n^{\text {th }}$ argument; likewise, $\partial_{n m}^{2} f$ will denote the second-order partial derivative with respect to the $n^{\text {th }}$ and $m^{\text {th }}$ arguments.
} 
with strict inequalities when $P\left(Q_{A}, Q_{B}\right)>0$.

(A.2) $P(0,0)>c$ and, for $Q$ sufficiently large, $P(0, Q)<c$.

Assumption (A.1) simply asserts that goods $A$ and $B$ are (imperfect) substitutes, whereas (A.2) is essentially a viability assumption. We now show that, under additional regularity conditions on demand, the three Cournot duopoly settings satisfy:

(P.1) In each of the three Cournot duopoly settings, there is a unique equilibrium, in which all quantities are positive; in addition, whenever a firm sells both goods, the goods are "internal" strategic substitutes. ${ }^{3}$

\section{A.1 Differentiated Monoproduct-Firm Cournot Duopoly}

In this section, we consider the setting in which one firm can only produce good $A$, and the other firm can only produce good $B$. To ensure the existence and uniqueness of an equilibrium, we introduce the following assumption:

(A.3) For any $\left(Q_{i}, Q_{j}\right) \geq 0$ such that $P\left(Q_{i}, Q_{j}\right)>0$, we have

$$
2 \partial_{1} P\left(Q_{i}, Q_{j}\right)+\partial_{11}^{2} P\left(Q_{i}, Q_{j}\right) Q_{i}<\partial_{2} P\left(Q_{i}, Q_{j}\right)+\partial_{12}^{2} P\left(Q_{i}, Q_{j}\right) Q_{i}<0
$$

Assumption (A.3) ensures that profit functions are concave and that firms' best responses have a slope that lies between -1 and 0 ; that is, firms' output decisions are strategic substitutes, and the resulting equilibrium is stable. In the case of linear demand, (A.3) boils down to $\partial_{1} P<\partial_{2} P<0$, and is thus implied by (A.1). Together with the previous assumptions, it ensures that the differentiated monoproduct-firm Cournot duopoly satisfies Property (P.1):

Proposition 10 Consider the differentiated monoproduct-firm Cournot duopoly in which firm 1, say, can produce good $A$ at constant unit cost $c$, and firm 2 can produce good $B$ at the same unit cost c. Under Assumptions (A.1) - (A.3), there exists a unique equilibrium. The equilibrium quantities $\left(q_{A 1}^{* *}, q_{B 2}^{* *}\right)$ are moreover symmetric, positive and characterized by the firstorder conditions: $q_{A 1}^{* *}=q_{B 2}^{* *}=Q^{* *}>0$, where $Q^{* *}$ is the unique solution to

$$
Q^{* *} \in \arg \max _{Q}\left[P\left(Q, Q^{* *}\right)-c\right] Q
$$

\footnotetext{
${ }^{3}$ That is, the profit $\pi$ of a firm, as a function of its quantities $q_{A}$ and $q_{B}$, is such that $\partial^{2} \pi / \partial q_{A} \partial q_{B} \leq 0$, with a strict inequality whenever the price of at least one good is positive.
} 
and is uniquely characterized by the first-order condition:

$$
P\left(Q^{* *}, Q^{* *}\right)-c+\partial_{1} P\left(Q^{* *}, Q^{* *}\right) Q^{* *}=0 .
$$

Proof. The equilibrium quantities are solutions to, for $i \neq j \in\{A, B\}$ :

$$
Q_{i}^{* *} \in \arg \max _{Q_{i}}\left[P\left(Q_{i}, Q_{j}^{* *}\right)-c\right] Q_{i} .
$$

From (A.1), the profit of the producer of good $i$ is strictly concave as long as the price of good $i$ is positive, and its best-response, $Q_{i}=\hat{\chi}\left(Q_{j}\right)$, satisfies the first-order condition

$$
\Psi\left(Q_{i}, Q_{j}\right)\left\{\begin{array}{lll}
\leq 0 & \text { if } & Q_{i}=0 \\
=0 & \text { if } & Q_{i}>0
\end{array}\right.
$$

where

$$
\Psi\left(Q_{i}, Q_{j}\right) \equiv P\left(Q_{i}, Q_{j}\right)-c+\partial_{1} P\left(Q_{i}, Q_{j}\right) Q_{i}
$$

We have $\Psi\left(0, Q_{j}\right)=P\left(0, Q_{j}\right)-c, \partial_{1} \Psi\left(Q_{i}, Q_{j}\right)=2 \partial_{1} P\left(Q_{i}, Q_{j}\right)+\partial_{11}^{2} P\left(Q_{i}, Q_{j}\right) Q_{i} \leq 0$ (with strict inequality if $\left.P\left(Q_{i}, Q_{j}\right)>0\right)$ by $(\mathbf{A . 1})$, and $\Psi\left(Q_{i}, Q_{j}\right)<0$ for $Q_{i}$ sufficiently large by (A.2). Hence, the best-response $\hat{\chi}\left(Q_{j}\right)$ is given by $\hat{\chi}\left(Q_{j}\right)=0$ if $P\left(0, Q_{j}\right) \leq c$, and by the unique solution to $\Psi\left(\hat{\chi}\left(Q_{j}\right), Q_{j}\right)=0$ otherwise. Furthermore, from (A.2), $\Psi(0,0)>0$ and $\Psi(Q, 0)<0$ for $Q$ sufficiently large. Therefore, $\hat{\chi}(0)>0$. Finally, as long as $\hat{\chi}(Q)>0$, we have:

$$
\hat{\chi}^{\prime}\left(Q_{j}\right)=-\frac{\partial_{2} \Psi\left(Q_{i}, Q_{j}\right)}{\partial_{1} \Psi\left(Q_{i}, Q_{j}\right)}=-\frac{\partial_{2} P\left(Q_{i}, Q_{j}\right)+\partial_{12}^{2} P\left(Q_{i}, Q_{j}\right) Q_{i}}{2 \partial_{1} P\left(Q_{i}, Q_{j}\right)+\partial_{11}^{2} P\left(Q_{i}, Q_{j}\right) Q_{i}}
$$

which by $(\mathbf{A . 3})$ satisfies $-1<\hat{\chi}^{\prime}(\cdot)<0$. It follows there there exists a unique equilibrium, which is moreover symmetric and involves positive quantities: $Q_{A}^{* *}=Q_{B}^{* *}=Q^{* *}$, the unique solution to $\Psi\left(Q^{* *}, Q^{* *}\right)=0$.

\section{A.2 Multiproduct-Firm Cournot Duopoly}

In this section, we consider the setting in which both firms can produce both goods. To ensure the existence and uniqueness of an equilibrium in this setting, we introduce the following assumption:

(A.4) For any $\left(Q_{i}, Q_{j}\right) \geq 0$ such that $P\left(Q_{i}, Q_{j}\right)>0$, and for any $q_{i} \in\left[0, Q_{i}\right]$ and any $q_{j} \in\left[0, Q_{j}\right]$, we have

$$
\begin{aligned}
& 2 \partial_{1} P\left(Q_{i}, Q_{j}\right)+\partial_{11}^{2} P\left(Q_{i}, Q_{j}\right) q_{i}+\partial_{22}^{2} P\left(Q_{j}, Q_{i}\right) q_{j} \\
< & \partial_{2} P\left(Q_{i}, Q_{j}\right)+\partial_{2} P\left(Q_{j}, Q_{i}\right)+\partial_{12}^{2} P\left(Q_{i}, Q_{j}\right) q_{i}+\partial_{12}^{2} P\left(Q_{j}, Q_{i}\right) q_{j} \\
< & 0 .
\end{aligned}
$$


Assumption (A.4) further guarantees that profits remain concave when both firms can sell goods $A$ and $B$. In the case of linear demand, it boils down to $\partial_{1} P<\partial_{2} P<0$, and is thus implied by (A.1). Together with the previous assumptions, it ensure that the multiproduct-firm Cournot duopoly satisfies Property (P.1):

Proposition 11 Consider the multiproduct-firm Cournot duopoly in which both firms can produce both goods $A$ and $B$ at constant unit cost c. Under Assumptions (A.1)-(A.4), the two goods are internal strategic substitutes, and there exists a unique equilibrium. The equilibrium quantities $\left(q_{A 1}^{\circ}, q_{A 2}^{\circ}, q_{B 1}^{\circ}, q_{B 2}^{\circ}\right)$ are moreover positive and symmetric: for $i \in\{A, B\}$ and $h \in\{1,2\}$, $q_{i h}^{\circ}=q^{\circ}>0$, where $q^{\circ}$ is the unique solution to

$$
q^{\circ} \in \arg \max _{q}\left[P\left(q+q^{\circ}, 2 q^{\circ}\right)-c\right] q+\left[P\left(2 q^{\circ}, q+q^{\circ}\right)-c\right] q^{\circ}
$$

and is uniquely characterized by the first-order condition:

$$
P\left(2 q^{\circ}, 2 q^{\circ}\right)-c+\left[\partial_{1} P\left(2 q^{\circ}, 2 q^{\circ}\right)+\partial_{2} P\left(2 q^{\circ}, 2 q^{\circ}\right)\right] q^{\circ}=0 .
$$

Proof. The equilibrium quantities are solutions to, for $h \neq k \in\{1,2\}$ :

$$
\left(q_{A h}^{\circ}, q_{B h}^{\circ}\right) \in \arg \max _{q_{A h}, q_{B h}} \Pi_{h}\left(q_{A h}, q_{B h} ; q_{A k}^{\circ}, q_{B k}^{\circ}\right)
$$

with

$$
\Pi_{h}\left(q_{A h}, q_{B h} ; q_{A k}, q_{B k}\right) \equiv\left[P\left(Q_{A}, Q_{B}\right)-c\right] q_{A h}+\left[P\left(Q_{B}, Q_{A}\right)-c\right] q_{B h},
$$

where $Q_{i} \equiv q_{i 1}+q_{i 2}$ denotes the total output for good $i$. The second-order derivatives of $\Pi_{h}(\cdot, \cdot ; \cdot, \cdot)$ are given by:

$$
\begin{aligned}
\partial_{11}^{2} \Pi_{h}\left(q_{A h}, q_{B h} ; q_{A k}, q_{B k}\right)= & 2 \partial_{1} P\left(q_{A}+q_{A k}, q_{B}+q_{B k}\right) \\
& +\partial_{11}^{2} P\left(q_{A}+q_{A k}, q_{B}+q_{B k}\right) q_{A}+\partial_{22}^{2} P\left(q_{B}+q_{B k}, q_{A}+q_{A k}\right) q_{B}, \\
\partial_{22}^{2} \Pi_{h}\left(q_{A h}, q_{B h} ; q_{A k}, q_{B k}\right)= & 2 \partial_{1} P\left(q_{B}+q_{B k}, q_{A}+q_{A k}\right) \\
& +\partial_{11}^{2} P\left(q_{B}+q_{B k}, q_{A}+q_{A k}\right) q_{B}+\partial_{22}^{2} P\left(q_{A}+q_{A k}, q_{B}+q_{B k}\right) q_{A}, \\
\partial_{12}^{2} \Pi_{h}\left(q_{A h}, q_{B h} ; q_{A k}, q_{B k}\right)= & \partial_{2} P\left(q_{A}+q_{A k}, q_{B}+q_{B k}\right)+\partial_{2} P\left(q_{B}+q_{B k}, q_{A}+q_{A k}\right) \\
& +\partial_{12}^{2} P\left(q_{A}+q_{A k}, q_{B}+q_{B k}\right) q_{A}+\partial_{12}^{2} P\left(q_{B}+q_{B k}, q_{A}+q_{A k}\right) q_{B} .
\end{aligned}
$$

It follows from (A.4) that:

- $\Pi_{h}\left(q_{A h}, q_{B h} ; \cdot, \cdot\right)$ is strictly concave in $\left(q_{A h}, q_{B h}\right)$; first-order conditions are therefore necessary and sufficient. 
- $\partial_{12}^{2} \Pi_{h}\left(q_{A h}, q_{B h} ; \cdot, \cdot\right) \leq 0$, with a strict inequality when the price of at least one good is positive; hence, goods $A$ and $B$ are internal strategic substitutes.

We first show that all quantities are positive, and then show that first-order conditions characterize a unique equilibrium.

- All equilibrium quantities are positive.

Suppose by contradiction that $q_{B 2}^{\circ}$, say, is zero.

Step 1: $q_{B 1}^{\circ}>0$. Suppose otherwise that $q_{B 1}^{\circ}=0$. By construction, we then have:

$$
q_{A h}^{\circ}=\arg \max _{q_{A h}}\left[P\left(q_{A h}+q_{A k}^{\circ}, 0\right)-c\right] q_{A h} .
$$

Note that $q_{A 1}^{\circ}=0$ would imply $p_{A}^{\circ}=P\left(q_{A 2}^{\circ}, 0\right) \leq c$, and thus $q_{A 2}^{\circ}=0$ as well; ${ }^{4}$ but this would therefore require $P(0,0) \leq c$, contradicting the viability condition (A.2). Thus, we can assume that $q_{A 1}^{\circ}$ is positive, and thus satisfies firm 1's first-order condition which, using

$$
\pi_{1}=\left[P\left(q_{A 1}+q_{A 2}^{\circ}, q_{B 1}\right)-c\right] q_{A 1}+\left[P\left(q_{B 1}, q_{A 1}+q_{A 2}^{\circ}\right)-c\right] q_{B 1},
$$

and $q_{B 1}^{\circ}=0$, is given by:

$$
\left.\frac{\partial \pi_{1}}{\partial q_{A 1}}\right|_{\left(q_{i h}\right)=\left(q_{i h}^{\circ}\right)}=P\left(Q_{A}^{\circ}, 0\right)-c+\partial_{1} P\left(Q_{A}^{\circ}, 0\right) q_{A 1}^{\circ}=0 .
$$

But then, a small increase in $q_{B 1}$ would increase firm 1's profit:

$$
\begin{aligned}
\left.\frac{\partial \pi_{1}}{\partial_{q_{B}}}\right|_{\left(q_{i h}\right)=\left(q_{i h}^{\circ}\right)} & =P\left(0, Q_{A}^{\circ}\right)-c+\partial_{2} P\left(Q_{A}^{\circ}, 0\right) q_{A 1}^{\circ} \\
& >P\left(Q_{A}^{\circ}, 0\right)-c+\partial_{1} P\left(Q_{A}^{\circ}, 0\right) q_{A 1}^{\circ}=0,
\end{aligned}
$$

where the inequality stems from (A.1) $\left(\partial_{2} P>\partial_{1} P\right.$, which also implies $P(0, Q)>P(Q, 0)$ for any $Q>0)$.

Step 2: $q_{A 2}^{\circ}>q_{A 1}^{\circ}$. From Step 1, $q_{B 1}^{\circ}$ is positive and therefore satisfies the first-order condition

$$
\left.\frac{\partial \pi_{1}}{\partial_{q_{B 1}}}\right|_{\left(q_{i h}\right)=\left(q_{i h}^{\circ}\right)}=P\left(Q_{B}^{\circ}, Q_{A}^{\circ}\right)-c+\partial_{1} P\left(Q_{B}^{\circ}, Q_{A}^{\circ}\right) q_{B 1}^{\circ}+\partial_{2} P\left(Q_{A}^{\circ}, Q_{B}^{\circ}\right) q_{A 1}^{\circ}=0 .
$$

From (A.1), $\partial_{1} P \leq 0$ and $\partial_{2} P \leq 0$ and thus $P\left(Q_{B}^{\circ}, Q_{A}^{\circ}\right) \geq c>0$; but (A.1) then implies $\partial_{1} P\left(Q_{B}^{\circ}, Q_{A}^{\circ}\right)<0$, which in turn yields $P\left(Q_{B}^{\circ}, Q_{A}^{\circ}\right)>c$.

${ }^{4}$ Otherwise, a slight reduction in $q_{A 2}$ would increase $R_{2}$ 's profit $\pi_{2}^{\circ}=\left(p_{A}^{\circ}-c\right) q_{A 2}^{\circ}$, since then

$$
\left.\frac{\partial \pi_{2}}{\partial q_{A 2}}\right|_{\left(q_{i h}\right)=\left(q_{i h}^{\circ}\right)}=p_{A}^{\circ}-c+q_{A 2}^{\circ} \partial_{1} P\left(q_{A 2}^{\circ}, 0\right)<p_{A}^{\circ}-c \leq 0 .
$$


Using

$$
\pi_{2}=\left[P\left(q_{A 1}^{\circ}+q_{A 2}, q_{B 1}^{\circ}+q_{B 2}\right)-c\right] q_{A 2}+\left[P\left(q_{B 1}^{\circ}+q_{B 2}, q_{A 1}^{\circ}+q_{A 2}\right)-c\right] q_{B 2},
$$

the first-order condition for $q_{B 2}^{\circ}=0$ yields:

$$
\left.\frac{\partial \pi_{2}}{\partial_{q_{B 2}}}\right|_{\left(q_{i h}\right)=\left(q_{i h}^{\circ}\right)}=P\left(Q_{B}^{\circ}, Q_{A}^{\circ}\right)-c+\partial_{2} P\left(Q_{A}^{\circ}, Q_{B}^{\circ}\right) q_{A 2}^{\circ} \leq 0 .
$$

Using (A.1) and $P\left(Q_{B}^{\circ}, Q_{A}^{\circ}\right)>c$, it follows that $q_{A 2}^{\circ}$ is positive and thus satisfies the first-order condition

$$
\left.\frac{\partial \pi_{2}}{\partial_{q_{A 2}}}\right|_{\left(q_{i h}\right)=\left(q_{i h}^{\circ}\right)}=P\left(Q_{A}^{\circ}, Q_{B}^{\circ}\right)-c+\partial_{1} P\left(Q_{A}^{\circ}, Q_{B}^{\circ}\right) q_{A 2}^{\circ}=0
$$

implying $P\left(Q_{A}^{\circ}, Q_{B}^{\circ}\right)>c$.

Subtracting (2) from (3) yields:

$$
\partial_{2} P\left(Q_{A}^{\circ}, Q_{B}^{\circ}\right)\left(q_{A 2}^{\circ}-q_{A 1}^{\circ}\right) \leq \partial_{1} P\left(Q_{B}^{\circ}, Q_{A}^{\circ}\right) q_{B 1}^{\circ},
$$

where $\partial_{1} P_{A}<0$ and $\partial_{2} P_{B}<0$ (from (A.1), as both prices are positive), and $q_{B 1}^{\circ}>0$ (from Step 1); therefore, $q_{A 2}^{\circ}>q_{A 1}^{\circ}$.

Step 3: $q_{A 1}^{\circ}>0$. Suppose otherwise that $q_{A 1}^{\circ}=0$. In that case, $Q_{A}^{\circ}=q_{A 2}^{\circ}$ and $Q_{B}^{\circ}=q_{B 1}^{\circ}$ satisfy $Q_{B}^{\circ}=\hat{\chi}\left(Q_{A}^{\circ}\right)$ and $Q_{A}^{\circ}=\hat{\chi}\left(Q_{B}^{\circ}\right)$, where the best response function

$$
\hat{\chi}(Q) \equiv \arg \max _{\hat{Q}}\{[P(\hat{Q}, Q)-c] \hat{Q}\}
$$

is characterized by the first-order condition:

$$
P(\hat{\chi}(Q), Q)-c+\partial_{1} P(\hat{\chi}(Q), Q) \hat{\chi}(Q)=0
$$

Assumption (A.3) ensures that this response function satisfies

$$
-1<\hat{\chi}^{\prime}(Q)<0
$$

Therefore, we must have $Q_{A}^{\circ}=Q_{B}^{\circ}=\hat{Q}^{\circ}$, where $\hat{Q}^{\circ}$ is such that $\hat{Q}^{\circ}=\hat{\chi}\left(\hat{Q}^{\circ}\right)$, and thus satisfies:

$$
P\left(\hat{Q}^{\circ}, \hat{Q}^{\circ}\right)-c+\partial_{1} P\left(\hat{Q}^{\circ}, \hat{Q}^{\circ}\right) \hat{Q}^{\circ}=0 .
$$

But then, each firm would want to sell the other good as well:

$$
\left.\frac{\partial \pi_{1}}{\partial_{q_{A 1}}}\right|_{\left(q_{i h}\right)=\left(q_{i h}^{\circ}\right)}=\left.\frac{\partial \pi_{2}}{\partial_{q_{B 2}}}\right|_{\left(q_{i h}\right)=\left(q_{i h}^{\circ}\right)}=P\left(\hat{Q}^{\circ}, \hat{Q}^{\circ}\right)-c+\partial_{2} P\left(\hat{Q}^{\circ}, \hat{Q}^{\circ}\right) \hat{Q}^{\circ}>0
$$


as $P\left(\hat{Q}^{\circ}, \hat{Q}^{\circ}\right)>c$ from above, and thus $\partial_{1} P\left(\hat{Q}^{\circ}, \hat{Q}^{\circ}\right)<\partial_{2} P\left(\hat{Q}^{\circ}, \hat{Q}^{\circ}\right)<0$ from (A.1). Hence, $q_{A 1}^{\circ}>0$.

Step 4. It follows from the previous steps that $q_{A 2}^{\circ}, q_{A 1}^{\circ}$ and $q_{B 1}^{\circ}$ must all be positive, and thus satisfy the first-order conditions:

$$
\begin{aligned}
& \left.\frac{\partial \pi_{1}}{\partial_{q_{A 1}}}\right|_{\left(q_{i h}\right)=\left(q_{i h}^{\circ}\right)}=P\left(Q_{A}^{\circ}, Q_{B}^{\circ}\right)-c+\partial_{1} P\left(Q_{A}^{\circ}, Q_{B}^{\circ}\right) q_{A 1}^{\circ}+\partial_{2} P\left(Q_{B}^{\circ}, Q_{A}^{\circ}\right) q_{B 1}^{\circ}=0, \\
& \left.\frac{\partial \pi_{1}}{\partial_{q_{B 1}}}\right|_{\left(q_{i h}\right)=\left(q_{i h}^{\circ}\right)}=P\left(Q_{B}^{\circ}, Q_{A}^{\circ}\right)-c+\partial_{1} P\left(Q_{B}^{\circ}, Q_{A}^{\circ}\right) q_{B 1}^{\circ}+\partial_{2} P\left(Q_{A}^{\circ}, Q_{B}^{\circ}\right) q_{A 1}^{\circ}=0, \\
& \left.\frac{\partial \pi_{2}}{\partial_{q_{A 2}}}\right|_{\left(q_{i h}\right)=\left(q_{i h}^{\circ}\right)}=P\left(Q_{A}^{\circ}, Q_{B}^{\circ}\right)-c+\partial_{1} P\left(Q_{A}^{\circ}, Q_{B}^{\circ}\right) q_{A 2}^{\circ}=0,
\end{aligned}
$$

whereas the first-order condition for $q_{B 2}^{\circ}=0$ yields:

$$
\left.\frac{\partial \pi_{2}}{\partial_{q_{B 2}}}\right|_{\left(q_{i h}\right)=\left(q_{i h}^{\circ}\right)}=P\left(Q_{B}^{\circ}, Q_{A}^{\circ}\right)-c+\partial_{2} P\left(Q_{A}^{\circ}, Q_{B}^{\circ}\right) q_{A 2}^{\circ} \leq 0 .
$$

Subtracting (6) from (4) and (5) from (7) yields:

$$
\begin{aligned}
& -\partial_{1} P\left(Q_{A}^{\circ}, Q_{B}^{\circ}\right)\left(q_{A 2}^{\circ}-q_{A 1}^{\circ}\right)=-\partial_{2} P\left(Q_{B}^{\circ}, Q_{A}^{\circ}\right) q_{B 1}^{\circ}, \\
& -\partial_{2} P\left(Q_{A}^{\circ}, Q_{B}^{\circ}\right)\left(q_{A 2}^{\circ}-q_{A 1}^{\circ}\right) \geq-\partial_{1} P\left(Q_{B}^{\circ}, Q_{A}^{\circ}\right) q_{B 1}^{\circ} .
\end{aligned}
$$

The first condition yields $q_{A 2}^{\circ}>q_{A 1}^{\circ}$, and thus the two conditions can be rewritten as:

$$
\frac{-\partial_{1} P\left(Q_{A}^{\circ}, Q_{B}^{\circ}\right)}{-\partial_{2} P\left(Q_{B}^{\circ}, Q_{A}^{\circ}\right)}=\frac{q_{B 1}^{\circ}}{q_{A 2}^{\circ}-q_{A 1}^{\circ}} \leq \frac{-\partial_{2} P\left(Q_{A}^{\circ}, Q_{B}^{\circ}\right)}{-\partial_{1} P\left(Q_{B}^{\circ}, Q_{A}^{\circ}\right)} .
$$

This, in turn, implies

$$
\partial_{1} P\left(Q_{A}^{\circ}, Q_{B}^{\circ}\right) \partial_{1} P\left(Q_{B}^{\circ}, Q_{A}^{\circ}\right) \leq \partial_{2} P\left(Q_{B}^{\circ}, Q_{A}^{\circ}\right) \partial_{2} P\left(Q_{A}^{\circ}, Q_{B}^{\circ}\right),
$$

a contradiction as $\partial_{1} P<\partial_{2} P<0$ from (A.1). Hence, there is no equilibrium in which $q_{B 2}^{\circ}=0$.

- The equilibrium exists, is unique and symmetric.

It follows from the above analysis that all equilibrium quantities are positive and thus satisfy the first-order conditions. Adding the conditions for good $A$, namely:

$$
\begin{aligned}
& \left.\frac{\partial \pi_{1}}{\partial_{q_{A 1}}}\right|_{\left(q_{i h}\right)=\left(q_{i h}^{\circ}\right)}=P\left(Q_{A}^{\circ}, Q_{B}^{\circ}\right)-c+\partial_{1} P\left(Q_{A}^{\circ}, Q_{B}^{\circ}\right) q_{A 1}^{\circ}+\partial_{2} P\left(Q_{B}^{\circ}, Q_{A}^{\circ}\right) q_{B 1}^{\circ}=0, \\
& \left.\frac{\partial \pi_{2}}{\partial_{q_{A 2}}}\right|_{\left(q_{i h}\right)=\left(q_{i h}^{\circ}\right)}=P\left(Q_{A}^{\circ}, Q_{B}^{\circ}\right)-c+\partial_{1} P\left(Q_{A}^{\circ}, Q_{B}^{\circ}\right) q_{A 2}^{\circ}+\partial_{2} P\left(Q_{B}^{\circ}, Q_{A}^{\circ}\right) q_{B 2}^{\circ}=0,
\end{aligned}
$$

implies that

$$
P\left(Q_{A}^{\circ}, Q_{B}^{\circ}\right) \geq c>0
$$


and $\left(Q_{A}^{\circ}, Q_{B}^{\circ}\right)$ satisfy $\phi\left(Q_{A}^{\circ}, Q_{B}^{\circ}\right)=0$, where

$$
\phi\left(Q_{A}, Q_{B}\right) \equiv 2\left[P\left(Q_{A}, Q_{B}\right)-c\right]+\partial_{1} P\left(Q_{A}, Q_{B}\right) Q_{A}+\partial_{2} P\left(Q_{B}, Q_{A}\right) Q_{B} .
$$

The derivatives of $\phi$ are given by:

$$
\begin{aligned}
& \partial_{1} \phi\left(Q_{A}, Q_{B}\right)=3 \partial_{1} P\left(Q_{A}, Q_{B}\right)+\partial_{11} P\left(Q_{A}, Q_{B}\right) Q_{A}+\partial_{22} P\left(Q_{B}, Q_{A}\right) Q_{B}, \\
& \partial_{2} \phi\left(Q_{A}, Q_{B}\right)=2 \partial_{2} P\left(Q_{A}, Q_{B}\right)+\partial_{2} P\left(Q_{B}, Q_{A}\right)+\partial_{12}^{2} P\left(Q_{A}, Q_{B}\right) Q_{A}+\partial_{21} P\left(Q_{B}, Q_{A}\right) Q_{B} .
\end{aligned}
$$

Assumptions $(\mathbf{A . 1})^{5}$ and (A.4) ensure that, as long as $P\left(Q_{A}, Q_{B}\right)>0$ :

$$
\partial_{1} \phi\left(Q_{A}, Q_{B}\right)<\partial_{2} \phi\left(Q_{A}, Q_{B}\right)<0 \text {. }
$$

In addition, Assumption (A.2) implies that $\phi(0,0)>0$ and Assumptions (A.1) and (A.2) jointly imply that, for any $Q_{B} \geq 0, \phi\left(Q_{A}, Q_{B}\right)<0$ for $Q_{A}$ sufficiently large. Therefore, for any $Q_{B}$ satisfying $\phi\left(0, Q_{B}\right)>0$, the condition $\phi\left(Q_{A}, Q_{B}\right)=0$ defines a unique "aggregate fitting-in" function, $Q_{A}=\tilde{\chi}\left(Q_{B}\right)$, which has moreover a slope strictly comprised between -1 and 0.

The above analysis shows that the equilibrium quantities satisfy $Q_{A}^{\circ}=\tilde{\chi}\left(Q_{B}^{\circ}\right)$. Likewise, adding the first-order conditions for good $B$ yields $P\left(Q_{B}^{\circ}, Q_{A}^{\circ}\right) \geq c>0$ and $Q_{B}^{\circ}=\tilde{\chi}\left(Q_{A}^{\circ}\right)$. As equilibrium quantities must be positive, it follows that there exists a unique equilibrium, which is symmetric and such that $Q_{A}^{\circ}=Q_{B}^{\circ}=Q^{\circ}=\tilde{\chi}\left(Q^{\circ}\right)>0$.

Finally, the first-order conditions for the quantity chosen by firm $h \in\{1,2\}$ yield:

$$
\begin{aligned}
& -\partial_{1} P\left(Q^{\circ}, Q^{\circ}\right) q_{A h}^{\circ}-\partial_{2} P\left(Q^{\circ}, Q^{\circ}\right) q_{B h}^{\circ}=P\left(Q^{\circ}, Q^{\circ}\right)-c, \\
& -\partial_{1} P\left(Q^{\circ}, Q^{\circ}\right) q_{B h}^{\circ}-\partial_{2} P\left(Q^{\circ}, Q^{\circ}\right) q_{A h}^{\circ}=P\left(Q^{\circ}, Q^{\circ}\right)-c .
\end{aligned}
$$

Furthermore, $Q^{\circ}$ satisfies $P\left(Q_{A}^{\circ}, Q_{B}^{\circ}\right) \geq c>0$ and $\phi\left(Q^{\circ}, Q^{\circ}\right)=0$, and thus:

$$
P\left(Q^{\circ}, Q^{\circ}\right)=c-\frac{\partial_{1} P\left(Q^{\circ}, Q^{\circ}\right) Q^{\circ}+\partial_{2} P\left(Q_{B}, Q_{A}\right) Q_{B}}{2}>c .
$$

Therefore, the individual quantities are also uniquely defined and symmetric:

$$
q_{A h}^{\circ}=q_{B h}^{\circ}=q^{\circ} \equiv-\frac{P\left(Q^{\circ}, Q^{\circ}\right)-c}{\partial_{1} P\left(Q^{\circ}, Q^{\circ}\right)+\partial_{2} P\left(Q^{\circ}, Q^{\circ}\right)}>0 .
$$

\footnotetext{
${ }^{5}$ Using demand symmetry (see footnote 1), (A.1) implies $\partial_{1} P\left(Q_{A}, Q_{B}\right)<\partial_{2} P\left(Q_{A}, Q_{B}\right)=\partial_{2} P\left(Q_{B}, Q_{A}\right)$.
} 


\section{A.3 Asymmetric Cournot Duopoly}

In this section, we consider the setting in which one firm can still produce both goods, whereas the other firm can only produce good $A$. To ensure the existence and uniqueness of an equilibrium in this setting, we introduce the following additional assumption:

(A.5) For any $\left(Q_{i}, Q_{j}\right) \geq 0$ such that $P\left(Q_{i}, Q_{j}\right)>0$, and for any $q_{j} \in\left[0, Q_{j}\right]$, we have

$$
\begin{aligned}
& 2 \partial_{1} P\left(Q_{i}, Q_{j}\right)+\partial_{11}^{2} P\left(Q_{i}, Q_{j}\right) Q_{i}+\partial_{22}^{2} P\left(Q_{j}, Q_{i}\right)\left(Q_{j}-q_{j}\right) \\
& +\partial_{2} P\left(Q_{j}, Q_{i}\right) \frac{\partial_{2} P\left(Q_{j}, Q_{i}\right)+\partial_{12}^{2} P\left(Q_{j}, Q_{i}\right) q_{j}}{\partial_{1} P\left(Q_{j}, Q_{i}\right)} \\
< & \partial_{2} P\left(Q_{i}, Q_{j}\right)+\partial_{12}^{2} P\left(Q_{i}, Q_{j}\right) Q_{i}+\partial_{12}^{2} P\left(Q_{j}, Q_{i}\right)\left(Q_{j}-q_{j}\right) \\
& +\partial_{2} P\left(Q_{j}, Q_{i}\right)\left(1+\frac{\partial_{1} P\left(Q_{j}, Q_{i}\right)+\partial_{11}^{2} P\left(Q_{j}, Q_{i}\right) q_{j}}{\partial_{1} P\left(Q_{j}, Q_{i}\right)}\right) \\
< & 0 .
\end{aligned}
$$

In the case of linear demand, (A.5) boils down to $2 \partial_{1} P+\left(\partial_{2} P\right)^{2} / \partial_{1} P<3 \partial_{2} P<0$, and is thus implied by (A.1). ${ }^{6}$ Together with the previous assumptions, it ensures that the asymmetric Cournot duopoly satisfies Property (P.1):

Proposition 12 Consider the asymmetric Cournot duopoly in which firm 1, say, produces both goods $A$ and $B$ at constant unit cost $c$, whereas firm 2 only produces good $B$, at the same unit cost c. Under Assumptions (A.1)-(A.5), the two goods are internal strategic substitutes for firm 1 , and there exists a unique equilibrium. The equilibrium quantities $\left(q_{A 1}^{*}, q_{B 1}^{*}, q_{B 2}^{*}\right)$ are moreover positive and uniquely characterized by the first-order conditions:

$$
\begin{aligned}
P\left(Q_{A}^{*}, Q_{B}^{*}\right)-c+\partial_{1} P\left(Q_{A}^{*}, Q_{B}^{*}\right) q_{A 1}^{*}+\partial_{2} P\left(Q_{A}^{*}, Q_{B}^{*}\right) q_{B 1}^{*} & =0, \\
P\left(Q_{B}^{*}, Q_{A}^{*}\right)-c+\partial_{1} P\left(Q_{B}^{*}, Q_{A}^{*}\right) q_{B 1}^{*}+\partial_{2} P\left(Q_{B}^{*}, Q_{A}^{*}\right) q_{A 1}^{*} & =0, \\
P\left(Q_{B}^{*}, Q_{A}^{*}\right)-c+\partial_{1} P\left(Q_{B}^{*}, Q_{A}^{*}\right) q_{B 2}^{*} & =0 .
\end{aligned}
$$

Proof. The equilibrium quantities are solutions to:

$$
\left(q_{A 1}^{*}, q_{B 1}^{*}\right) \in \arg \max _{q_{A 1}, q_{B 1}}\left[P\left(q_{A 1}, q_{B 1}+q_{B 2}^{*}\right)-c\right] q_{A 1}+\left[P\left(q_{B 1}+q_{B 2}^{*}, q_{A 1}\right)-c\right] q_{B 1},
$$

${ }^{6}$ That $2 \partial_{1} P+\left(\partial_{2} P\right)^{2} / \partial_{1} P<0$ and $3 \partial_{2} P<0$ derives directly from (A.2). Furthermore:

$$
\left|2 \partial_{1} P+\frac{\left(\partial_{2} P\right)^{2}}{\partial_{1} P}\right|>3\left|\partial_{2} P\right| \Longleftrightarrow 3\left|\frac{\partial_{2} P}{\partial_{1} P}\right|<2+\left(\frac{\partial_{2} P}{\partial_{1} P}\right)^{2},
$$

where the last inequality holds for $\partial_{2} P / \partial_{1} P \in(0,1)$. 
and

$$
q_{B 2}^{*} \in \arg \max _{q_{B 2}}\left[P\left(q_{B 1}^{*}+q_{B 2}, q_{A 1}^{*}\right)-c\right] q_{B 2} .
$$

The profits of the two firms are again strictly concave from (A.4), and the first order conditions are given by:

$$
\begin{aligned}
& P\left(Q_{A}^{*}, Q_{B}^{*}\right)-c+\partial_{1} P\left(Q_{A}^{*}, Q_{B}^{*}\right) q_{A 1}^{*}+\partial_{2} P\left(Q_{A}^{*}, Q_{B}^{*}\right) q_{B 1}^{*} \leq 0, \leq 0 \\
& P\left(Q_{B}^{*}, Q_{A}^{*}\right)-c+\partial_{1} P\left(Q_{B}^{*}, Q_{A}^{*}\right) q_{B 1}^{*}+\partial_{2} P\left(Q_{B}^{*}, Q_{A}^{*}\right) q_{A 1}^{*} \leq 0 \\
& P\left(Q_{B}^{*}, Q_{A}^{*}\right)-c+\partial_{1} P\left(Q_{B}^{*}, Q_{A}^{*}\right) q_{B 2}^{*} \leq 0,
\end{aligned}
$$

where the first-order condition of quantity $q_{i k}^{*}$ holds with equality if $q_{i k}^{*}>0$, and where $Q_{A}^{*} \equiv q_{A 1}^{*}$ and $Q_{B}^{*} \equiv q_{B 1}^{*}+q_{B 2}^{*}$.

We first show that all quantities are positive, and then show that first-order conditions characterize a unique equilibrium.

- All equilibrium quantities are positive.

Step 1: $P\left(Q_{B}^{*}, Q_{A}^{*}\right)>c$. To see this, suppose otherwise that $P\left(Q_{B}^{*}, Q_{A}^{*}\right) \leq c$. Then, the first-order condition of $q_{B 2}^{*}$ implies that $q_{B 2}^{*}=0$. Moreover, we must also have $q_{B 1}^{*}=0$; if not, firm 1 could profitably deviate by reducing $q_{B 1}$. Hence, $Q_{B}^{*}=0 \leq Q_{A}^{*}$. By (A.1), we thus have $P\left(Q_{A}^{*}, Q_{B}^{*}\right) \leq c$, implying that $q_{A 1}^{*}=0$ (otherwise, firm 1 could profitably deviate by reducing $\left.q_{A 1}\right)$. Hence, $P(0,0) \leq c$, contradicting (A.2).

Step 2: $q_{B 2}^{*}>0$. As $P\left(Q_{B}^{*}, Q_{A}^{*}\right)>c$, firm 2 could otherwise profitably deviate by choosing $q_{B 2}>0$ small enough such that $P\left(Q_{B}^{*}+q_{B 2}, Q_{A}^{*}\right)>c$.

Step 3: $P\left(Q_{A}^{*}, Q_{B}^{*}\right)>c$. To see this, suppose otherwise that $P\left(Q_{A}^{*}, Q_{B}^{*}\right) \leq c$. It follows that $q_{A 1}^{*}=0$. (If not, firm 1 could profitably deviate by setting $q_{A 1}=0$; if $q_{B 1}^{*}=0$, firm 1 could combine this deviation with some $q_{B 1}>0$ sufficiently small such that $P\left(Q_{B}^{*}+q_{B 1}, Q_{A}^{*}-q_{A 1}^{*}\right)>c$. $)$ Hence, $Q_{A}^{*}=0 \leq Q_{B}^{*}$. By $(\mathbf{A} . \mathbf{1})$, we thus have $P\left(Q_{A}^{*}, Q_{B}^{*}\right) \geq P\left(Q_{B}^{*}, Q_{A}^{*}\right)>c$, a contradiction.

Step 4: $q_{A 1}^{*}>0$. Suppose otherwise that $q_{A 1}^{*}=0$. We have:

$$
\begin{aligned}
0 & \geq P\left(Q_{A}^{*}, Q_{B}^{*}\right)-c+\partial_{2} P\left(Q_{A}^{*}, Q_{B}^{*}\right) q_{B 1}^{*} \\
& >P\left(Q_{B}^{*}, Q_{A}^{*}\right)-c+\partial_{1} P\left(Q_{B}^{*}, Q_{A}^{*}\right) q_{B 1}^{*},
\end{aligned}
$$

where the first inequality follows from $q_{A 1}^{*}=0$, and the second by $(\mathbf{A . 1})$ and $Q_{B}^{*}>Q_{A}^{*}$. It follows that $q_{B 1}^{*}=0$. But as $P\left(Q_{B}^{*}, Q_{A}^{*}\right)>c$, firm 1 could then profitably deviate by setting $q_{B 1}>0$ sufficiently small such that $P\left(Q_{B}^{*}+q_{B 1}, Q_{A}^{*}\right)>c$.

Step 5: $q_{B 1}^{*}>0$. Suppose otherwise that $q_{B 1}^{*}=0$. The induced outcome thus coincides with the equilibrium outcome in a duopoly in which firm 1 sells only good $A$ and firm 2 sells only 
good B. Under Assumption (A.3), this implies that $Q_{A}^{*}=Q_{B}^{*}$, as shown in Proposition 10. We thus have:

$$
P\left(Q_{B}^{*}, Q_{A}^{*}\right)-c+\partial_{2} P\left(Q_{B}^{*}, Q_{A}^{*}\right) Q_{A}^{*}>P\left(Q_{A}^{*}, Q_{B}^{*}\right)-c+\partial_{1} P\left(Q_{A}^{*}, Q_{B}^{*}\right) Q_{A}^{*}=0,
$$

where the inequality follows from (A.1) and the equality from the first-order condition of $q_{A 1}^{*}=$ $Q_{A}^{*}$. But then firm 1 could profitably deviate by slightly raising $q_{B 1}$.

- The equilibrium exists and is unique.

All quantities $\left(q_{A 1}^{*}, q_{B 1}^{*}, q_{B 2}^{*}\right)$ are therefore strictly positive, implying that each of the three first-order conditions holds with equality; that is (using $Q_{A}^{*}=q_{A 1}^{*}$ ):

$$
\begin{aligned}
P\left(Q_{A}^{*}, Q_{B}^{*}\right)-c+\partial_{1} P\left(Q_{A}^{*}, Q_{B}^{*}\right) Q_{A}^{*}+\partial_{2} P\left(Q_{B}^{*}, Q_{A}^{*}\right) q_{B 1}^{*}=0, \\
P\left(Q_{B}^{*}, Q_{A}^{*}\right)-c+\partial_{1} P\left(Q_{B}^{*}, Q_{A}^{*}\right) q_{B 1}^{*}+\partial_{2} P\left(Q_{A}^{*}, Q_{B}^{*}\right) Q_{A}^{*}=0, \\
P\left(Q_{B}^{*}, Q_{A}^{*}\right)-c+\partial_{1} P\left(Q_{B}^{*}, Q_{A}^{*}\right) q_{B 2}^{*}=0 .
\end{aligned}
$$

Adding equations (10) and (11), we obtain $\phi\left(Q_{B}^{*}, Q_{A}^{*}\right)=0$, where the function $\phi$ is defined by (8). Therefore, $Q_{B}^{*}=\tilde{\chi}\left(Q_{A}^{*}\right)$.

Equation (10) yields $q_{B 2}^{*}=q_{B 2}\left(Q_{A}^{*}, Q_{B}^{*}\right)$, where:

$$
q_{B 2}\left(Q_{A}, Q_{B}\right) \equiv \min \left\{\max \left\{-\frac{P\left(Q_{B}, Q_{A}\right)-c}{\partial_{1} P\left(Q_{B}, Q_{A}\right)}, 0\right\}, Q_{B}\right\},
$$

which is continuous in $Q_{A}$ and $Q_{B}$, and such that:

$$
\begin{aligned}
& \partial_{1} q_{B 2}\left(Q_{A}, Q_{B}\right)=-\frac{\partial_{2} P\left(Q_{B}, Q_{A}\right)+\partial_{12}^{2} P\left(Q_{B}, Q_{A}\right) q_{B 2}\left(Q_{A}, Q\right)}{\partial_{1} P\left(Q_{B}, Q_{A}\right)} \\
& \partial_{2} q_{B 2}\left(Q_{A}, Q_{B}\right)=-\frac{\partial_{1} P\left(Q_{B}, Q_{A}\right)+\partial_{11}^{2} P\left(Q_{B}, Q_{A}\right) q_{B 2}\left(Q_{A}, Q_{B}\right)}{\partial_{1} P\left(Q_{B}, Q_{A}\right)}
\end{aligned}
$$

whenever $q_{B 2}($.$) lies strictly between 0$ and $Q_{B}$. Plugging this into (9) yields $\phi_{A}\left(Q_{A}^{*}, Q_{B}^{*}\right)=0$, where:

$$
\phi_{A}\left(Q_{A}, Q_{B}\right)=P\left(Q_{A}, Q_{B}\right)-c+\partial_{1} P\left(Q_{A}, Q_{B}\right) Q_{A}+\partial_{2} P\left(Q_{B}, Q_{A}\right)\left(Q_{B}-q_{B 2}\left(Q_{A}, Q_{B}\right)\right) .
$$

Whenever $q_{B 2}($.$) is differentiable (which is almost everywhere), \phi_{A}$ is also differentiable and its derivatives are given by:

$$
\begin{aligned}
\partial_{1} \phi_{A}\left(Q_{A}^{*}, Q_{B}\right)= & 2 \partial_{1} P\left(Q_{A}, Q_{B}\right)+\partial_{11}^{2} P\left(Q_{A}, Q_{B}\right) Q_{A} \\
& +\partial_{22}^{2} P\left(Q_{B}, Q_{A}\right)\left(Q_{B}-q_{B 2}\left(Q_{A}, Q_{B}\right)\right)-\partial_{2} P\left(Q_{B}, Q_{A}\right) \partial_{1} q_{B 2}\left(Q_{A}, Q_{B}\right), \\
\partial_{2} \phi_{A}\left(Q_{A}, Q_{B}\right)= & \partial_{2} P\left(Q_{A}, Q_{B}\right)+\partial_{12}^{2} P\left(Q_{A}, Q_{B}\right) Q_{A} \\
& +\partial_{12}^{2} P\left(Q_{B}, Q_{A}\right)\left(Q_{B}-q_{B 2}\left(Q_{A}, Q_{B}\right)\right)+\partial_{2} P\left(Q_{B}, Q_{A}\right)\left(1-\partial_{2} q_{B 2}\left(Q_{A}, Q_{B}\right)\right) .
\end{aligned}
$$


Assumptions (A.3) (for the case when $\left.q_{B 2}(\cdot)=Q_{B}\right),(\mathbf{A . 4})\left(\right.$ when $\left.q_{B 2}(\cdot)=0\right)$ and $(\mathbf{A . 5})$ (when $\left.0<q_{B 2}(\cdot)<Q_{B}\right)$ ensure that, as long as $P\left(Q_{A}, Q_{B}\right)>0, \partial_{1} \phi_{A}\left(Q_{A}, Q_{B}\right)<\partial_{2} \phi_{A}\left(Q_{A}, Q_{B}\right)<0$. In addition, Assumption (A.2) implies that $\phi_{A}(0,0)>0$ and Assumptions (A.1) and (A.2) jointly imply that, for any $Q_{B} \geq 0, \phi_{A}\left(Q_{A}, Q_{B}\right)<0$ for $Q_{A}$ sufficiently large. Therefore, for any $Q_{B}$ satisfying $\phi_{A}\left(0, Q_{B}\right)>0$, the condition $\phi_{A}\left(Q_{A}, Q_{B}\right)=0$ defines a unique aggregate fitting-in function, $Q_{A}=\tilde{\chi}_{A}\left(Q_{B}\right)$, which has moreover a slope strictly comprised between -1 and 0 .

Summing-up, the above analysis shows that the equilibrium aggregate quantities satisfy $Q_{A}^{*}=\tilde{\chi}_{A}\left(Q_{B}^{*}\right)$ and $Q_{B}^{*}=\tilde{\chi}\left(Q_{A}^{*}\right)$, where $\tilde{\chi}($.$) and \tilde{\chi}_{A}($.$) each has a slope lying between -1$ and 0 . It follows that there exists a unique quantity pair $\left(Q_{A}^{*}, Q_{B}^{*}\right)$ such that $Q_{A}^{*}>0, Q_{B}^{*}>0$, and $Q_{A}^{*}=\tilde{\chi}_{A}\left(Q_{B}^{*}\right)$ and $Q_{B}^{*}=\tilde{\chi}\left(Q_{A}^{*}\right)$. As equilibrium quantities must be positive, these constitute the unique candidate equilibrium aggregate quantities. To conclude the proof, it suffices to show that individual quantities are also positive.

We first note that $\phi_{A}\left(Q_{A}^{*}, Q_{B}^{*}\right)=0$ and $\phi\left(Q_{B}^{*}, Q_{A}^{*}\right)=0$ respectively imply $P\left(Q_{A}^{*}, Q_{B}^{*}\right)>c$ and $P\left(Q_{B}^{*}, Q_{A}^{*}\right)>c$. It follows that $q_{B 2}^{*}=q_{B 2}\left(Q_{A}^{*}, Q_{B}^{*}\right)>0$. Finally, showing that $q_{B 1}^{*}>$ 0 amounts to establishing $q_{B 2}^{*}<Q_{B}^{*}$. Suppose by contradiction that $Q_{B}^{*}=q_{B 2}^{*}$. As $q_{B 2}^{*}=$ $q_{B 2}\left(Q_{A}^{*}, Q_{B}^{*}\right)$, this requires:

$$
P\left(Q_{B}^{*}, Q_{A}^{*}\right)-c+\partial_{1} P\left(Q_{B}^{*}, Q_{A}^{*}\right) Q_{B}^{*} \geq 0 .
$$

Combining this with $Q_{B}^{*}=\tilde{\chi}\left(Q_{A}^{*}\right)$, or $\phi\left(Q_{B}^{*}, Q_{A}^{*}\right)=0$, this yields:

$$
\begin{aligned}
P\left(Q_{B}^{*}, Q_{A}^{*}\right)-c+\partial_{2} P\left(Q_{A}^{*}, Q_{B}^{*}\right) Q_{A}^{*} & \leq 0 \\
& =P\left(Q_{A}^{*}, Q_{B}^{*}\right)-c+\partial_{1} P\left(Q_{A}^{*}, Q_{B}^{*}\right) Q_{A}^{*} \\
& <P\left(Q_{A}^{*}, Q_{B}^{*}\right)-c+\partial_{2} P\left(Q_{A}^{*}, Q_{B}^{*}\right) Q_{A}^{*},
\end{aligned}
$$

where the equality stems from $\phi_{A}\left(Q_{A}^{*}, Q_{B}^{*}\right)=0$ (using $q_{B 2}^{*}=Q_{B}^{*}$ ) and the strict inequality stems from $Q_{A}^{*}>0$ and (A.1). It follows that

$$
P\left(Q_{A}^{*}, Q_{B}^{*}\right)>P\left(Q_{B}^{*}, Q_{A}^{*}\right)
$$

and thus

$$
Q_{A}^{*}<Q_{B}^{*}
$$


But then, we obtain a contradiction to (13), as:

$$
\begin{aligned}
0 & \leq P\left(Q_{B}^{*}, Q_{A}^{*}\right)-c+\partial_{1} P\left(Q_{B}^{*}, Q_{A}^{*}\right) Q_{B}^{*} \\
& <P\left(Q_{B}^{*}, Q_{A}^{*}\right)-c+\partial_{2} P\left(Q_{B}^{*}, Q_{A}^{*}\right) Q_{A}^{*} \\
& =P\left(Q_{B}^{*}, Q_{A}^{*}\right)-c+\partial_{2} P\left(Q_{A}^{*}, Q_{B}^{*}\right) Q_{A}^{*}
\end{aligned}
$$

where the first inequality comes from (12), the strict inequality stems from $Q_{A}^{*}<Q_{B}^{*}$ and (A.1), and the equality derives from $\partial_{2} P\left(Q_{B}, Q_{A}\right)=\partial_{2} P\left(Q_{A}, Q_{B}\right)$.

\section{B Property (P.2)}

We conclude this Online Appendix by showing that the Cournot duopoly games introduced in Section 3 of the paper satisfy Property (P.2) under an additional regularity conditions on demand, namely:

(B.4) For any $\left(Q_{i}, Q_{j}\right) \geq 0$ such that $P\left(Q_{i}, Q_{j}\right)>0$, we have

$$
\begin{array}{r}
\partial_{2} P\left(Q_{i}, Q_{j}\right)\left[\partial_{11}^{2} P\left(Q_{i}, Q_{j}\right) Q_{i}+\partial_{22}^{2} P\left(Q_{j}, Q_{i}\right) Q_{j}\right] \\
\geq \quad \partial_{1} P\left(Q_{i}, Q_{j}\right)\left[\partial_{12}^{2} P\left(Q_{i}, Q_{j}\right) Q_{i}+\partial_{12}^{2} P\left(Q_{j}, Q_{i}\right) Q_{j}\right] .
\end{array}
$$

This condition is satisfied when for instance demand is linear. We have:

Proposition 13 The three Cournot duopoly settings considered in Section A satisfy the following properties:

- Under Assumptions (A.1) - (A.5) and (B.1) - (B.3), the equilibrium industry profits are such that: $\Pi^{* *}>\Pi^{*}>\Pi^{\circ}$.

- Under Assumptions (A.1) - (A.4), the equilibrium aggregate outputs are such that: $Q^{* *}<$ $Q^{\circ}$.

- Under Assumptions (A.1) - (A.5) and (B.1) - (B.2), a monoproduct firm sells less when its rival produces both goods (asymmetric duopoly) than when it produces a single good (differentiated monoproduct-firm duopoly): $q_{B 1}^{*}>q_{B 1}^{* *}$

Proof. To establish the first result, we start by noting that, from Proposition 12, Assumptions (A.1) - (A.5) imply $q_{B 1}^{*}>0$. Therefore:

$$
\Pi^{* *}=\tilde{\Pi}\left(q_{B 1}^{*}, 0\right)>\tilde{\Pi}(0,0)=\Pi^{*},
$$


where the inequality follows from (Г.3), which, from Proposition 15, holds under (B.1) - (B.3). It thus suffices to show that $\Pi^{*}>\Pi^{\circ}$.

Let $\left(\hat{Q}_{A}(\hat{c}), \hat{Q}_{B}(\hat{c})\right)$ denote the equilibrium outputs of the hypothetical duopoly game $\hat{\Gamma}$ introduced in Section 5.3 of the paper, and studied in Section C of this Online Appendix, in which firm 1 can produce both goods at $\operatorname{cost} c$, whereas firm 2 can produce good $A$ at cost $c$ and $\operatorname{good} B$ at cost $\hat{c}>c$. As noted in the proof of Proposition $7,\left(Q^{\circ}, Q^{\circ}\right)=\left(\hat{Q}_{A}(c), \hat{Q}_{B}(c)\right)$ and $\left(Q_{A}^{*}, Q_{B}^{*}\right)=\left(\hat{Q}_{A}\left(\hat{c}^{*}\right), \hat{Q}_{B}\left(\hat{c}^{*}\right)\right)$, where

$$
\hat{c}^{*} \equiv P\left(Q_{A}^{*}, Q_{B}^{*}\right)+\partial_{2} P\left(Q_{B}^{*}, Q_{A}^{*}\right) q_{B 2}^{*} .
$$

is the cost level which leads firm 2 to stop selling good $A$. We can thus interpret the move from $\left(Q^{\circ}, Q^{\circ}\right)$ to $\left(Q_{A}^{*}, Q_{B}^{*}\right)$ as the evolution of the equilibrium $\left(\hat{Q}_{A}(\hat{c}), \hat{Q}_{B}(\hat{c})\right)$ as $\hat{c}$ increases from $c$ to $\hat{c}^{*}$. Furthermore, for $\hat{c}=\hat{c}^{*}, q_{A 2}^{*}=0$ and thus the industry profit in the duopoly game coincides with the "true" industry profit, based on the actual cost $c$ :

$$
\Pi^{*}=\hat{\Pi}\left(\hat{c}^{*}\right),
$$

where

$$
\hat{\Pi}(\hat{c}) \equiv\left(P\left(\hat{Q}_{A}(\hat{c}), \hat{Q}_{B}(\hat{c})\right)-c\right) \hat{Q}_{A}(\hat{c})+\left(P\left(\hat{Q}_{B}(\hat{c}), \hat{Q}_{A}(\hat{c})\right)-c\right) \hat{Q}_{B}(\hat{c})
$$

denotes the equilibrium industry profit, based on true costs, in the duopoly game $\hat{\Gamma}$. As by construction $\Pi^{\circ}=\hat{\Pi}(c)$, to establish $\Pi^{*}>\Pi^{\circ}$, it suffices to show that $\hat{\Pi}(\hat{c})$ increases with $\hat{c}$.

We have:

$$
\begin{aligned}
\hat{\Pi}^{\prime}(\hat{c})= & {\left[P\left(\hat{Q}_{A}, \hat{Q}_{B}\right)-c+\partial_{1} P\left(\hat{Q}_{A}, \hat{Q}_{B}\right) \hat{Q}_{A}+\partial_{2} P\left(\hat{Q}_{B}, \hat{Q}_{A}\right) \hat{Q}_{B}\right] \hat{Q}_{A}^{\prime} } \\
& +\left[P\left(\hat{Q}_{B}, \hat{Q}_{A}\right)-c+\partial_{1} P\left(\hat{Q}_{B}, \hat{Q}_{A}\right) \hat{Q}_{B}+\partial_{2} P\left(\hat{Q}_{A}, \hat{Q}_{B}\right) \hat{Q}_{A}\right] \hat{Q}_{B}^{\prime},
\end{aligned}
$$

which, using the FOCs for $R_{1}$ 's outputs $\hat{q}_{A 1}$ and $\hat{q}_{B 1}$ :

$$
\begin{aligned}
& P\left(\hat{Q}_{B}, \hat{Q}_{A}\right)-c+\partial_{1} P\left(\hat{Q}_{B}, \hat{Q}_{A}\right) \hat{q}_{B 1}+\partial_{2} P\left(\hat{Q}_{A}, \hat{Q}_{B}\right) \hat{q}_{A 1}=0 \\
& P\left(\hat{Q}_{A}, \hat{Q}_{B}\right)-c+\partial_{1} P\left(\hat{Q}_{A}, \hat{Q}_{B}\right) \hat{q}_{A 1}+\partial_{2} P\left(\hat{Q}_{B}, \hat{Q}_{A}\right) \hat{q}_{B 1}=0
\end{aligned}
$$

can be written as:

$$
\begin{aligned}
\hat{\Pi}^{\prime}(\hat{c})= & {\left[\partial_{1} P\left(\hat{Q}_{A}, \hat{Q}_{B}\right) \hat{q}_{A 2}+\partial_{2} P\left(\hat{Q}_{B}, \hat{Q}_{A}\right) \hat{q}_{B 2}\right] \hat{Q}_{A}^{\prime} } \\
& +\left[\partial_{1} P\left(\hat{Q}_{B}, \hat{Q}_{A}\right) \hat{q}_{B 2}+\partial_{2} P\left(\hat{Q}_{A}, \hat{Q}_{B}\right) \hat{q}_{A 2}\right] R^{\prime}\left(\hat{Q}_{A}\right) \hat{Q}_{A}^{\prime} \\
= & \left\{\begin{array}{c}
{\left[\partial_{1} P\left(\hat{Q}_{A}, \hat{Q}_{B}\right)+\partial_{2} P\left(\hat{Q}_{A}, \hat{Q}_{B}\right) R^{\prime}\left(\hat{Q}_{A}\right)\right] \hat{q}_{A 2}} \\
+\left[\partial_{2} P\left(\hat{Q}_{B}, \hat{Q}_{A}\right)+\partial_{1} P\left(\hat{Q}_{B}, \hat{Q}_{A}\right) R^{\prime}\left(\hat{Q}_{A}\right)\right] \hat{q}_{B 2}
\end{array}\right\} \hat{Q}_{A}^{\prime} .
\end{aligned}
$$


The first term within bracket is negative, as $\partial_{1} P\left(\hat{Q}_{A}, \hat{Q}_{B}\right)<\partial_{2} P\left(\hat{Q}_{A}, \hat{Q}_{B}\right)<0$ and $R^{\prime}\left(\hat{Q}_{A}\right)>$ -1 . Under (B.4), the aggregate best-response $Q_{B}=R\left(Q_{A}\right)$ satisfies:

$$
\partial_{2} P\left(Q_{B}, Q_{A}\right)+\partial_{1} P\left(Q_{B}, Q_{A}\right) R^{\prime}\left(Q_{A}\right) \leq 0
$$

Hence, the second term within brackets is non-positive, as $\hat{Q}_{A}^{\prime}<0$. It follows that $\hat{\Pi}^{\prime}(\hat{c})>0$.

We now turn to the second result. Recall that $Q^{* *}=q^{* *}$ and $Q^{\circ}=2 q^{\circ}$ are such that

$$
\begin{aligned}
Q^{* *} & =\arg \max _{Q}\left[P\left(Q, Q^{* *}\right)-c\right] Q, \\
\frac{Q^{\circ}}{2} & =\arg \max _{q}\left[P\left(\frac{Q^{\circ}}{2}+q, Q^{\circ}\right)-c\right] q+\left[P\left(Q^{\circ}, \frac{Q^{\circ}}{2}+q\right)-c\right] \frac{Q^{\circ}}{2} .
\end{aligned}
$$

Hence, we have

$$
\left[P\left(Q^{* *}, Q^{* *}\right)-c\right] Q^{* *} \geq\left[P\left(Q^{\circ}, Q^{* *}\right)-c\right] Q^{\circ}
$$

and

$$
\begin{aligned}
{\left[P\left(Q^{\circ}, Q^{\circ}\right)-c\right] Q^{\circ} } & \geq\left[P\left(Q^{* *}, Q^{\circ}\right)-c\right]\left(Q^{* *}-\frac{Q^{\circ}}{2}\right)+\left[P\left(Q^{\circ}, Q^{* *}\right)-c\right] \frac{Q^{\circ}}{2} \\
& =\left[P\left(Q^{* *}, Q^{\circ}\right)-c\right] Q^{* *}+\left[P\left(Q^{\circ}, Q^{* *}\right)-P\left(Q^{* *}, Q^{\circ}\right)\right] \frac{Q^{\circ}}{2}
\end{aligned}
$$

If $Q^{* *}>Q^{\circ}$, the last term on the RHS is positive from (A.2), implying

$$
\left[P\left(Q^{\circ}, Q^{\circ}\right)-c\right] Q^{\circ} \geq\left[P\left(Q^{* *}, Q^{\circ}\right)-c\right] Q^{* *}
$$

Combining (15) and (16) yields

$$
\left[P\left(Q^{* *}, Q^{* *}\right)-P\left(Q^{* *}, Q^{\circ}\right)\right] Q^{* *} \geq\left[P\left(Q^{\circ}, Q^{* *}\right)-P\left(Q^{\circ}, Q^{\circ}\right)\right] Q^{\circ}
$$

i.e.,

which is equivalent to

$$
\int_{Q^{\circ}}^{Q^{* *}} Q^{* *} \partial_{2} P\left(Q^{* *}, Q\right) d Q \geq \int_{Q^{\circ}}^{Q^{* *}} Q^{\circ} \partial_{2} P\left(Q^{\circ}, Q\right) d Q
$$

$$
\int_{Q^{\circ}}^{Q^{* *}} \int_{Q^{\circ}}^{Q^{* *}}\left[\partial_{2} P(\tilde{Q}, Q)+\tilde{Q} \partial_{12}^{2} P(\tilde{Q}, Q)\right] d \tilde{Q} d Q \geq 0 .
$$

(A.3) implies that the term in brackets is negative, a contradiction. Hence, we must have $Q^{* *} \leq Q^{\circ}$.

Suppose now for contradiction that $Q^{* *}=Q^{\circ}$. The first-order conditions of the above maximization problems (for $q^{* *}=Q^{* *}$ and $q^{\circ}=Q^{\circ} / 2$ ) then yield:

$$
P\left(Q^{\circ}, Q^{\circ}\right)-c=-\left[\partial_{1} P\left(Q^{\circ}, Q^{\circ}\right)+\partial_{2} P\left(Q^{\circ}, Q^{\circ}\right)\right] \frac{Q^{\circ}}{2}=-\partial_{1} P\left(Q^{\circ}, Q^{\circ}\right) Q^{\circ},
$$


implying $\partial_{1} P\left(Q^{\circ}, Q^{\circ}\right)=\partial_{2} P\left(Q^{\circ}, Q^{\circ}\right)$, and thus contradicting (A.2). Hence, we must have $Q^{* *}<Q^{\circ}$.

Finally the last result follows from Proposition 12, which shows that Assumptions (A.1) (A.5) imply $q_{B 1}^{*}>0$, and from Proposition 15 , which shows that $(\boldsymbol{\Gamma} . \mathbf{2})$ holds under $(\mathbf{B . 1})-(\mathbf{B . 2})$; therefore:

$$
q_{B 2}^{*}=\tilde{q}_{B 2}(0,0)>\tilde{q}_{B 2}\left(q_{B 1}^{*}, 0\right)=q_{B 2}^{* *} .
$$

\section{Game $\hat{\Gamma}$}

We consider the hypothetical duopoly game, introduced in Section 5.3 of the paper, and which we will refer to as Game $\hat{\Gamma}$, in which firm 1 , say, produces both goods at cost $c$, whereas the other firm produces one good $-\operatorname{good} A$, say - at cost $\hat{c} \geq c$, and the other good at cost $c$. We provide a condition on demand ensuring that this game satisfies Property $(\mathbf{B})$.

As shown in the text, the first-order conditions allow us to define an aggregate best-response $Q_{A}=\hat{R}\left(Q_{B}, \hat{c}\right)$, implicitly characterized by the aggregate first-order condition

$$
2 P\left(Q_{A}, Q_{B}\right)-c-\hat{c}+\partial_{1} P\left(Q_{A}, Q_{B}\right) Q_{A}+\partial_{2} P\left(Q_{B}, Q_{A}\right) Q_{B}=0,
$$

and another aggregate best-response $Q_{B}=R\left(Q_{A}\right)$, implicitly characterized by

$$
2 P\left(Q_{B}, Q_{A}\right)-2 c+\partial_{1} P\left(Q_{B}, Q_{A}\right) Q_{B}+\partial_{2} P\left(Q_{A}, Q_{B}\right) Q_{A}=0 .
$$

We have:

Proposition 14 Under Assumptions (A.1) and (A.3):

- (B) For any $Q>0,-1<R^{\prime}(Q) \leq 0$, with a strict inequality when $R(Q)>0,-1<$ $\partial_{1} \hat{R}(Q, \hat{c}) \leq 0$, and $\partial_{2} \hat{R}(Q, \hat{c}) \leq 0$, with strict inequalities when $\hat{R}(Q, \hat{c})>0$.

Proof. Differentiating (17) with respect to $Q_{A}, Q_{B}$ and $\hat{c}$ yields

$$
\hat{\lambda}_{A} d Q_{A}+\hat{\mu}_{A} d Q_{B}=d \hat{c},
$$

where

$$
\begin{aligned}
& \hat{\lambda}_{i}=3 \partial_{1} P\left(\tilde{Q}_{i}, \tilde{Q}_{j}\right)+\tilde{Q}_{i} \partial_{11}^{2} P\left(\tilde{Q}_{i}, \tilde{Q}_{j}\right)+\tilde{Q}_{j} \partial_{22}^{2} P\left(\tilde{Q}_{j}, \tilde{Q}_{i}\right) \\
& \hat{\mu}_{i}=2 \partial_{2} P\left(\tilde{Q}_{i}, \tilde{Q}_{j}\right)+\partial_{2} P\left(\tilde{Q}_{j}, \tilde{Q}_{i}\right)+\tilde{Q}_{i} \partial_{12}^{2} P\left(\tilde{Q}_{i}, \tilde{Q}_{j}\right)+\tilde{Q}_{j} \partial_{12}^{2} P\left(\tilde{Q}_{j}, \tilde{Q}_{i}\right)
\end{aligned}
$$


The aggregate first-order condition (17) implies $P\left(Q_{A}, Q_{B}\right) \geq(c+\hat{c}) / 2>0$. Therefore, (A.1) and (A.3) together imply $\hat{\lambda}_{i}<\hat{\mu}_{i}<0$. We thus have

$$
-1<\partial_{1} \hat{R}\left(Q_{B}, \hat{c}\right)=-\frac{\hat{\mu}_{A}}{\hat{\lambda}_{A}}<0
$$

and

$$
\partial_{2} \hat{R}\left(Q_{B}, \hat{c}\right)=\frac{1}{\hat{\lambda}_{A}}<0
$$

Likewise, differentiating (18) with respect to $Q_{A}$ and $Q_{B}$ yields

$$
\hat{\lambda}_{B} d Q_{B}+\hat{\mu}_{B} d Q_{A}=0
$$

and a similar reasoning leads to

$$
-1<R^{\prime}\left(Q_{A}\right)=-\frac{\hat{\mu}_{B}}{\hat{\lambda}_{B}}<0
$$

\section{Game $\Gamma$}

We now consider game $\Gamma$, in which both firms can produce both goods $A$ and $B$, but the output levels $q_{B 1}$ and $q_{A 2}$ are exogenously fixed. That is, firm 1 only decides how much of good $A$ to sell, $q_{A 1}$, and firm 2 only chooses its quantity $q_{B 2}$ of good $B$; the profit functions of firms 1 and 2 are respectively given by

$$
\hat{\Pi}_{1}\left(q_{A 1}, q_{B 2} ; \hat{q}_{B 1}, \hat{q}_{A 2}\right) \equiv \Pi_{1}\left(q_{A 1}, \hat{q}_{B 1} ; \hat{q}_{A 2}, q_{B 2}\right)
$$

and

$$
\hat{\Pi}_{2}\left(q_{A 1}, q_{B 2} ; \hat{q}_{B 1}, \hat{q}_{A 2}\right) \equiv \Pi_{2}\left(\hat{q}_{A 2}, q_{B 2} ; q_{A 1}, \hat{q}_{B 1}\right) .
$$

In the special case where $\hat{q}_{A 2}=\hat{q}_{B 1}=0$, this game simplifies to the differentiated monoproductfirm Cournot duopoly studied in Subsection A.1, where each good is sold by only one firm.

To ensure that this game yields a well-behaved equilibrium, we introduce the following assumptions:

(B.1) For any $Q_{i}, Q_{j} \geq 0$ and for any $\left(q_{i}, q_{j}\right) \in\left[0, Q_{i}\right] \times\left[0, Q_{j}\right]$,

$$
\begin{aligned}
& 2 \partial_{1} P\left(Q_{i}, Q_{j}\right)+q_{i} \partial_{11}^{2} P\left(Q_{i}, Q_{j}\right)+q_{j} \partial_{22}^{2} P\left(Q_{j}, Q_{i}\right) \\
\leq & \partial_{2} P\left(Q_{i}, Q_{j}\right)+q_{i} \partial_{12}^{2} P\left(Q_{i}, Q_{j}\right)+q_{j} \partial_{12}^{2} P\left(Q_{j}, Q_{i}\right) \\
\leq & 0
\end{aligned}
$$

with strict inequalities when $P\left(Q_{i}, Q_{j}\right)>0$. 
(B.2) For any $Q_{i}, Q_{j} \geq 0$ and for any $q_{i} \in\left[0, Q_{i}\right]$,

$$
\begin{aligned}
& 2 \partial_{1} P\left(Q_{i}, Q_{j}\right)+q_{i} \partial_{11}^{2} P\left(Q_{i}, Q_{j}\right)+Q_{j} \partial_{22}^{2} P\left(Q_{j}, Q_{i}\right) \\
\leq & \partial_{2} P\left(Q_{i}, Q_{j}\right)+\partial_{2} P\left(Q_{j}, Q_{i}\right)+q_{i} \partial_{12}^{2} P\left(Q_{i}, Q_{j}\right)+Q_{j} \partial_{12}^{2} P\left(Q_{j}, Q_{i}\right),
\end{aligned}
$$

with strict inequalities when $P\left(Q_{i}, Q_{j}\right)>0$, and in addition, for any $q_{j} \in\left[0, Q_{j}\right]$,

$$
\begin{array}{r}
\partial_{1} P\left(Q_{i}, Q_{j}\right)\left[\begin{array}{c}
2 \partial_{1} P\left(Q_{j}, Q_{i}\right)-\partial_{2} P\left(Q_{j}, Q_{i}\right) \\
+q_{j}\left(\partial_{11}^{2} P\left(Q_{j}, Q_{i}\right)-\partial_{12}^{2} P\left(Q_{j}, Q_{i}\right)\right) \\
+q_{i}\left(\partial_{22}^{2} P\left(Q_{i}, Q_{j}\right)-\partial_{12}^{2} P\left(Q_{i}, Q_{j}\right)\right)
\end{array}\right] \\
\geq \partial_{2} P\left(Q_{i}, Q_{j}\right)\left[\begin{array}{c}
2 \partial_{1} P\left(Q_{i}, Q_{j}\right)-\partial_{2} P\left(Q_{i}, Q_{j}\right) \\
+\left[Q_{i}-q_{i}\right]\left(\partial_{11}^{2} P\left(Q_{i}, Q_{j}\right)-\partial_{12}^{2} P\left(Q_{i}, Q_{j}\right)\right) \\
+\left[Q_{j}-q_{j}\right]\left(\partial_{22}^{2} P\left(Q_{j}, Q_{i}\right)-\partial_{12}^{2} P\left(Q_{j}, Q_{i}\right)\right)
\end{array}\right],
\end{array}
$$

with strict inequalities when $P\left(Q_{i}, Q_{j}\right)>0$ and $P\left(Q_{j}, Q_{i}\right)>0$.

(B.3) For any $Q_{i}, Q_{j} \geq 0$ and for any $q_{i} \in\left[0, Q_{i}\right]$,

$$
\partial_{1} P\left(Q_{i}, Q_{j}\right)+q_{i} \partial_{11}^{2} P\left(Q_{i}, Q_{j}\right)+Q_{j} \partial_{22}^{2} P\left(Q_{j}, Q_{i}\right)<0 .
$$

with strict inequalities when $P\left(Q_{i}, Q_{j}\right)>0$, and in addition, for any $q_{j} \in\left[0, Q_{j}\right]$,

$$
\begin{array}{ccc}
{\left[\partial_{2} P_{i}+\partial_{2} P_{j}+\left(Q_{i}-q_{i}\right) \partial_{12}^{2} P_{i}+q_{j} \partial_{12}^{2} P_{j}\right] \leq} & {\left[2 \partial_{1} P_{i}+\left(Q_{i}-q_{i}\right) \partial_{11}^{2} P_{i}+q_{j} \partial_{22}^{2} P_{j}\right]} \\
\times\left[\partial_{2} P_{j}+\left(Q_{j}-q_{j}\right) \partial_{12}^{2} P_{j}+q_{i} \partial_{12}^{2} P_{i}\right] & \times\left[\partial_{1} P_{j}+\left(Q_{j}-q_{j}\right) \partial_{11}^{2} P_{j}+q_{i} \partial_{22}^{2} P_{i}\right],
\end{array}
$$

where $P_{i} \equiv P\left(Q_{i}, Q_{j}\right)$ and $P_{j} \equiv P\left(Q_{j}, Q_{i}\right)$, with a strict inequality when $P_{i}>0$ and $P_{j}>0$.

In the case of linear demand (B.1), (B.2) and (B.3) boil down to, respectively, $2 \partial_{1} P<$ $\partial_{2} P<0, \partial_{1} P<\partial_{2} P$ and $\left(\partial_{1} P-\partial_{2} P\right)\left(2 \partial_{1}-\partial_{2} P\right)>0$, and $\left(\partial_{2} P\right)^{2}<\left(\partial_{1} P\right)^{2}$; they are thus all implied by (A.1).

The following Proposition shows that these assumptions ensure that the game $\Gamma$ yields a well-behaved equilibrium:

Proposition 15 We have:

- Under Assumptions (A.1) and (B.1):

(Г.1) Game $\Gamma$ has a unique equilibrium. 
Let $\left(\tilde{q}_{A 1}\left(\hat{q}_{B 1}, \hat{q}_{A 2}\right), \tilde{q}_{B 2}\left(\hat{q}_{B 1}, \hat{q}_{A 2}\right)\right)$ denote the equilibrium quantities and $\tilde{\Pi}_{i}\left(\hat{q}_{B 1}, \hat{q}_{A 2}\right)$ denote firm $i$ 's equilibrium profit.

- Under Assumptions (A.1) and (B.1) - (B.2):

(Г.2) In game $\Gamma$, the aggregate equilibrium profit

$$
\tilde{\Pi}\left(\hat{q}_{B 1}, \hat{q}_{A 2}\right)=\tilde{\Pi}_{1}\left(\hat{q}_{B 1}, \hat{q}_{A 2}\right)+\tilde{\Pi}_{2}\left(\hat{q}_{B 1}, \hat{q}_{A 2}\right)
$$

is uniquely maximized for $\hat{q}_{B 1}=\hat{q}_{A 2}=0$; that is, $\tilde{\Pi}(0,0)>\tilde{\Pi}\left(\hat{q}_{B 1}, \hat{q}_{A 2}\right)$ whenever $\hat{q}_{B 1}+\hat{q}_{A 2}>0$.

- Under Assumptions (A.1) and (B.1) - (B.3):

(Г.3) In game $\Gamma$, the equilibrium quantity $\tilde{q}_{A 1}\left(\hat{q}_{A 2}, \hat{q}_{B 1}\right)\left(\right.$ resp., $\left.\tilde{q}_{B 2}\left(\hat{q}_{B 1}, \hat{q}_{A 2}\right)\right)$ is decreasing in $\hat{q}_{A 2}$ (resp., $\hat{q}_{B 1}$ ), and strictly so as long as it is positive.

Proof. We first consider property (Г.1), and assume that (A.1) and (B.1) hold. The derivative of firm $i$ 's profit, $\hat{\Pi}_{i}$, with respect to $q_{i h}$ is (for $i h \neq j k \in\{A 1, B 2\}$ ):

$\Phi\left(q_{i h} ; q_{j k}, \hat{q}_{j h}, \hat{q}_{i k}\right) \equiv P\left(q_{i h}+\hat{q}_{i k}, \hat{q}_{j h}+q_{j k}\right)-c+q_{i h} \partial_{1} P\left(q_{i h}+\hat{q}_{i k}, \hat{q}_{j h}+q_{j k}\right)+\hat{q}_{j h} \partial_{2} P\left(\hat{q}_{j h}+q_{j k}, q_{i h}+\hat{q}_{i k}\right)$, and is thus such that:

$\frac{d \Phi}{d q_{i h}}\left(q_{i h} ; q_{j k}, \hat{q}_{j h}, \hat{q}_{i k}\right)=2 \partial_{1} P\left(q_{i k}+\hat{q}_{i l}, \hat{q}_{j k}+q_{j l}\right)+q_{i k} \partial_{11}^{2} P\left(q_{i k}+\hat{q}_{i l}, \hat{q}_{j k}+q_{j l}\right)+\hat{q}_{j k} \partial_{22}^{2} P\left(\hat{q}_{j k}+q_{j l}, q_{i k}+\hat{q}_{i l}\right)$.

From (A.1), $\Phi\left(q_{i h} ; q_{j k}, \hat{q}_{j h}, \hat{q}_{i k}\right)=0$ implies $P\left(q_{i h}+\hat{q}_{i k}, \hat{q}_{j h}+q_{j k}\right) \geq c(>0)$, and thus, from (B.1), $\frac{d \Phi}{d q_{i h}}\left(q_{i h} ; q_{j k}, \hat{q}_{j h}, \hat{q}_{i k}\right)<0$; it follows that firm $i$ 's best-response

$$
r\left(q_{j k} ; \hat{q}_{B 1}, \hat{q}_{A 2}\right)=\arg \max _{q_{i h}} \hat{\Pi}_{i}\left(q_{A 1}, q_{B 2} ; \hat{q}_{B 1}, \hat{q}_{A 2}\right)
$$

is single-valued. It is moreover positive whenever

$$
P\left(\hat{q}_{i k}, \hat{q}_{j h}+q_{j k}\right)>c-\hat{q}_{j h} \partial_{2} P\left(\hat{q}_{j h}+q_{j k}, \hat{q}_{i k}\right),
$$

in which case it is characterized by the first-order condition $\Phi\left(q_{i h} ; q_{j k}, \hat{q}_{j h}, \hat{q}_{i k}\right)=0$. Differentiating this first-order condition with respect to $q_{i h}$ and $q_{j k}$ then yields:

$$
\frac{d r}{d q_{j k}}\left(q_{j k} ; \hat{q}_{j h}, \hat{q}_{i k}\right)=-\frac{\lambda_{i}}{\mu_{i}}
$$


where

$$
\begin{gathered}
2 \partial_{1} P\left(q_{i h}+\hat{q}_{i k}, \hat{q}_{j h}+q_{j k}\right) \\
\lambda_{i} \equiv+q_{i h} \partial_{11}^{2} P\left(q_{i h}+\hat{q}_{i k}, \hat{q}_{j h}+q_{j k}\right)+\hat{q}_{j h} \partial_{22}^{2} P\left(\hat{q}_{j h}+q_{j k}, q_{i h}+\hat{q}_{i k}\right) \\
\left.\mu_{i} \equiv \quad\right|_{q_{i h}=r\left(q_{j k} ; \hat{q}_{j h}, \hat{q}_{i k}\right)}, \\
+q_{i h} \partial_{12}^{2} P\left(q_{i h}+\hat{q}_{i k}, \hat{q}_{j h}+q_{j k}\right)+\hat{q}_{j h} \partial_{12}^{2} P\left(\hat{q}_{j h}+q_{j k}, q_{i h}+\hat{q}_{i k}\right)
\end{gathered} \mid
$$

As $\lambda_{i}<\mu_{i}<0$ from (B.1), (19) implies

$$
-1<\frac{d r}{d q_{j k}}<0
$$

Hence there exists a unique Nash equilibrium, which is moreover "stable" in the usual sense.

We now turn to property $(\boldsymbol{\Gamma} . \mathbf{2})$, and assume that $(\mathbf{A . 1})$ and $(\mathbf{B . 1})-(\mathbf{B . 2})$ hold. We consider three steps.

- Step 1: An increase in either $\hat{q}_{A 2}$ or $\hat{q}_{B 1}$ leads to a strict increase in the total equilibrium output $\tilde{Q}_{A}+\tilde{Q}_{B}$, where $\tilde{Q}_{i}$ denotes the total equilibrium output of good $i$ in game $\Gamma$.

This is obvious (although in a weak sense, for the "decreasing" part) when $\tilde{q}_{A 1}\left(\hat{q}_{B 1}, \hat{q}_{A 2}\right)=$ $\tilde{q}_{B 2}\left(\hat{q}_{B 1}, \hat{q}_{A 2}\right)=0$, as then $\tilde{Q}_{A}=\hat{q}_{A 2}$ and $\tilde{Q}_{B}=\hat{q}_{B 1}$. Consider now the case where $\tilde{q}_{i h}\left(\hat{q}_{B 1}, \hat{q}_{A 2}\right)>$ 0 whereas $\tilde{q}_{j k}\left(\hat{q}_{B 1}, \hat{q}_{A 2}\right)=0$, for $i h \neq j k \in\{A 1, B 2\}$. We then have $\tilde{Q}_{j}=\hat{q}_{j h}$, and thus $\frac{\partial \tilde{Q}_{j}}{\partial \hat{q}_{i k}}=0, \frac{\partial \tilde{Q}_{j}}{\partial \hat{q}_{j h}}=1$. Turning to $\tilde{Q}_{i}=\tilde{q}_{i h}+\hat{q}_{i k}$, the first-order condition for $\tilde{q}_{i h}$ is:

$$
P\left(\tilde{q}_{i h}+\hat{q}_{i k}, \hat{q}_{j h}\right)-c+\tilde{q}_{i h} \partial_{1} P\left(\tilde{q}_{i h}+\hat{q}_{i k}, \hat{q}_{j h}\right)+\hat{q}_{j h} \partial_{2} P\left(\hat{q}_{j h}, \tilde{q}_{i h}+\hat{q}_{i k}\right)=0,
$$

or:

$$
P\left(\tilde{Q}_{i}, \hat{q}_{j h}\right)-c+\tilde{Q}_{i} \partial_{1} P\left(\tilde{Q}_{i}, \hat{q}_{j h}\right)=\hat{q}_{i k} \partial_{1} P\left(\tilde{Q}_{i}, \hat{q}_{j h}\right)-\hat{q}_{j h} \partial_{2} P\left(\hat{q}_{j h}, \tilde{Q}_{i}\right) .
$$

Differentiating this equation with respect to $\tilde{Q}_{i}$ and $\hat{q}_{j k}$ yields, using $\tilde{Q}_{j}=\hat{q}_{j h}$ :

$$
\tilde{\lambda}_{i} d \tilde{Q}_{i}=\partial_{1} P\left(\tilde{Q}_{i}, \tilde{Q}_{j}\right) d \hat{q}_{i k}-\left[\partial_{2} P\left(\tilde{Q}_{j}, \tilde{Q}_{i}\right)+\tilde{\mu}_{i}\right] d \hat{q}_{j h}
$$

where

$$
\begin{aligned}
& \tilde{\lambda}_{i}=2 \partial_{1} P\left(\tilde{Q}_{i}, \tilde{Q}_{j}\right)+\tilde{q}_{i h} \partial_{11}^{2} P\left(\tilde{Q}_{i}, \tilde{Q}_{j}\right)+\tilde{Q}_{j} \partial_{22}^{2} P\left(\tilde{Q}_{j}, \tilde{Q}_{i}\right), \\
& \tilde{\mu}_{i}=\partial_{2} P\left(\tilde{Q}_{i}, \tilde{Q}_{j}\right)+\tilde{q}_{i h} \partial_{12}^{2} P\left(\tilde{Q}_{i}, \hat{q}_{j h}\right)+\tilde{Q}_{j} \partial_{12}^{2} P\left(\tilde{Q}_{j}, \tilde{Q}_{i}\right) .
\end{aligned}
$$

The first-order condition (20) implies $P_{i}=P\left(\tilde{Q}_{i}, \tilde{Q}_{j}\right) \geq c>0$, and thus Assumptions (A.1) and $(\mathbf{B . 2 a})$ respectively yield $\partial_{1} P\left(\tilde{Q}_{i}, \tilde{Q}_{j}\right)<\partial_{2} P\left(\tilde{Q}_{j}, \tilde{Q}_{i}\right)<0^{7}$ and $\tilde{\lambda}_{i}<\partial_{2} P\left(\tilde{Q}_{j}, \tilde{Q}_{i}\right)+\tilde{\mu}_{i}<$

\footnotetext{
${ }^{7}$ See footnote 5 .
} 
$\tilde{\mu}_{i}<0$; therefore:

$$
\begin{aligned}
\frac{\partial \tilde{Q}_{i}}{\partial \hat{q}_{i k}} & =\frac{\partial_{1} P\left(\tilde{Q}_{i}, \tilde{Q}_{j}\right)}{\tilde{\lambda}_{i}}>0 \\
-1 & <\frac{\partial \tilde{Q}_{i}}{\partial \hat{q}_{j h}}=-\frac{\partial_{2} P\left(\tilde{Q}_{j}, \tilde{Q}_{i}\right)+\tilde{\mu}_{i}}{\tilde{\lambda}_{i}}<0,
\end{aligned}
$$

which yields:

$$
\begin{aligned}
\frac{\partial\left(\tilde{Q}_{A}+\tilde{Q}_{B}\right)}{\partial \hat{q}_{i k}} & =\frac{\partial \tilde{Q}_{i}}{\partial \hat{q}_{i k}}=\frac{\partial_{1} P\left(\tilde{Q}_{i}, \tilde{Q}_{j}\right)}{\tilde{\lambda}_{i}}>0, \\
\frac{\partial\left(\tilde{Q}_{A}+\tilde{Q}_{B}\right)}{\partial \hat{q}_{j h}} & =1+\frac{\partial \tilde{Q}_{i}}{\partial \hat{q}_{j h}}=1-\frac{\partial_{2} P\left(\tilde{Q}_{j}, \tilde{Q}_{i}\right)+\tilde{\mu}_{i}}{\tilde{\lambda}_{i}}>0 .
\end{aligned}
$$

Let us now consider the case where $\tilde{q}_{A 1}, \tilde{q}_{B 2}>0$, and are thus characterized by the first-order conditions:

$P\left(\tilde{q}_{A 1}+\hat{q}_{A 2}, \hat{q}_{B 1}+\tilde{q}_{B 2}\right)-c+\tilde{q}_{A 1} \partial_{1} P\left(\tilde{q}_{A 1}+\hat{q}_{A 2}, \hat{q}_{B 1}+\tilde{q}_{B 2}\right)+\hat{q}_{B 1} \partial_{2} P\left(\hat{q}_{B 1}+\tilde{q}_{B 2}, \tilde{q}_{A 1}+\hat{q}_{A 2}\right)=0$, $P\left(\hat{q}_{B 1}+\tilde{q}_{B 2}, \tilde{q}_{A 1}+\hat{q}_{A 2}\right)-c+\tilde{q}_{B 2} \partial_{1} P\left(\hat{q}_{B 1}+\tilde{q}_{B 2}, \tilde{q}_{A 1}+\hat{q}_{A 2}\right)+\hat{q}_{A 2} \partial_{2} P\left(\tilde{q}_{A 1}+\hat{q}_{A 2}, \hat{q}_{B 1}+\tilde{q}_{B 2}\right)=0$, or, in terms of total equilibrium outputs $\tilde{Q}_{A}=\tilde{q}_{A 1}+\hat{q}_{A 2}$ and $\tilde{Q}_{B}=\hat{q}_{B 1}+\tilde{q}_{B 2}$ :

$$
\begin{aligned}
& P\left(\tilde{Q}_{A}, \tilde{Q}_{B}\right)-c+\tilde{Q}_{A} \partial_{1} P\left(\tilde{Q}_{A}, \tilde{Q}_{B}\right)=\hat{q}_{A 2} \partial_{1} P\left(\tilde{Q}_{A}, \tilde{Q}_{B}\right)-\hat{q}_{B 1} \partial_{2} P\left(\tilde{Q}_{B}, \tilde{Q}_{A}\right) \\
& P\left(\tilde{Q}_{B}, \tilde{Q}_{A}\right)-c+\tilde{Q}_{B} \partial_{1} P\left(\tilde{Q}_{B}, \tilde{Q}_{A}\right)=\hat{q}_{B 1} \partial_{1} P\left(\tilde{Q}_{B}, \tilde{Q}_{A}\right)-\hat{q}_{A 2} \partial_{2} P\left(\tilde{Q}_{A}, \tilde{Q}_{B}\right)
\end{aligned}
$$

which implies $P\left(\tilde{Q}_{i}, \tilde{Q}_{j}\right), P\left(\tilde{Q}_{j}, \tilde{Q}_{i}\right) \geq c>0$. Furthermore, differentiating these equations with respect to $\left(\tilde{Q}_{A}, \tilde{Q}_{B}\right)$ and $\left(\hat{q}_{A 2}, \hat{q}_{B 1}\right)$ yields:

$$
\begin{aligned}
& \tilde{\lambda}_{A} d \tilde{Q}_{A}+\tilde{\mu}_{A} d \tilde{Q}_{B}=\partial_{1} P\left(\tilde{Q}_{A}, \tilde{Q}_{B}\right) d \hat{q}_{A 2}-\partial_{2} P\left(\tilde{Q}_{B}, \tilde{Q}_{A}\right) d \hat{q}_{B 1}, \\
& \tilde{\mu}_{B} d \tilde{Q}_{A}+\tilde{\lambda}_{B} d \tilde{Q}_{B}=-\partial_{2} P\left(\tilde{Q}_{A}, \tilde{Q}_{B}\right) d \hat{q}_{A 2}+\partial_{1} P\left(\tilde{Q}_{B}, \tilde{Q}_{A}\right) d \hat{q}_{B 1},
\end{aligned}
$$

where $\tilde{\lambda}_{i}$ and $\tilde{\mu}_{i}$ are now given by (with $\left.i h \neq j k \in\{A 1, B 1\}\right)$ :

$$
\begin{aligned}
& \tilde{\lambda}_{i}=2 \partial_{1} P\left(\tilde{Q}_{i}, \tilde{Q}_{j}\right)+\tilde{q}_{i h} \partial_{11}^{2} P\left(\tilde{Q}_{i}, \tilde{Q}_{j}\right)+\hat{q}_{j h} \partial_{22}^{2} P\left(\tilde{Q}_{j}, \tilde{Q}_{i}\right), \\
& \tilde{\mu}_{i}=\partial_{2} P\left(\tilde{Q}_{i}, \tilde{Q}_{j}\right)+\tilde{q}_{i h} \partial_{12}^{2} P\left(\tilde{Q}_{i}, \tilde{Q}_{j}\right)+\hat{q}_{j h} \partial_{12}^{2} P\left(\tilde{Q}_{j}, \tilde{Q}_{i}\right) .
\end{aligned}
$$

Under Assumption (B.1), these coefficients satisfy $\tilde{\lambda}_{i}<\tilde{\mu}_{i}$; the determinant $D=\tilde{\lambda}_{A} \tilde{\lambda}_{B}-\tilde{\mu}_{A} \tilde{\mu}_{B}$ is therefore positive, and thus:

$$
\begin{aligned}
\frac{\partial \tilde{Q}_{i}}{\partial \hat{q}_{i k}} & =\frac{\tilde{\lambda}_{j} \partial_{1} P\left(\tilde{Q}_{i}, \tilde{Q}_{j}\right)+\tilde{\mu}_{i} \partial_{2} P\left(\tilde{Q}_{i}, \tilde{Q}_{j}\right)}{D} \\
\frac{\partial \tilde{Q}_{j}}{\partial \hat{q}_{i k}} & =-\frac{\tilde{\mu}_{j} \partial_{1} P\left(\tilde{Q}_{i}, \tilde{Q}_{j}\right)+\tilde{\lambda}_{i} \partial_{2} P\left(\tilde{Q}_{i}, \tilde{Q}_{l}\right)}{D} .
\end{aligned}
$$


Therefore, we have:

$$
\frac{\partial\left(\tilde{Q}_{A}+\tilde{Q}_{B}\right)}{\partial \hat{q}_{i k}}=\frac{\left(\tilde{\lambda}_{j}-\tilde{\mu}_{j}\right) \partial_{1} P\left(\tilde{Q}_{i}, \tilde{Q}_{j}\right)-\left(\tilde{\lambda}_{i}-\tilde{\mu}_{i}\right) \partial_{2} P\left(\tilde{Q}_{i}, \tilde{Q}_{j}\right)}{D} .
$$

Assumption (B.2b) ensures that the numerator, too, is positive, which concludes the proof of this step.

- Step 2: The aggregate profit $\Pi(Q, Q)=2[P(Q, Q)-c] Q$ is strictly decreasing in $Q$ for all $Q \geq Q^{* *}$ such that $P(Q, Q)>0$, where $Q^{* *}$ is the equilibrium quantity of each good in the duopoly with monoproduct firms considered in Section A.1.

This is obvious when $Q$ is so large that $P(Q, Q)=0$. When instead $P(Q, Q)>0$, then the derivative of the aggregate profit with respect to per-good output $Q$ is

$$
\frac{d \Pi(Q, Q)}{d Q}=2\left[P(Q, Q)-c+Q \partial_{1} P(Q, Q)+Q \partial_{2} P(Q, Q)\right]
$$

We have:

$$
\left.\frac{d \Pi(Q, Q)}{d Q}\right|_{Q=Q^{* *}}=2 Q^{* *} \partial_{2} P\left(Q^{* *}, Q^{* *}\right)<0,
$$

where the inequality stems from (A.1). In addition:

$$
\begin{aligned}
\frac{1}{2} \frac{d^{2} \Pi(Q, Q)}{d Q^{2}}= & \frac{d}{d Q}\left[P(Q, Q)-c+Q \partial_{1} P(Q, Q)+Q \partial_{2} P(Q, Q)\right] \\
= & {\left[2 \partial_{1} P(Q, Q)+Q \partial_{11} P(Q, Q)+Q \partial_{22} P(Q, Q)\right] } \\
& +\left[\partial_{2} P(Q, Q)+Q\left(\partial_{12}^{2} P(Q, Q)+\partial_{12}^{2} P(Q, Q)\right)\right]+\partial_{2} P(Q, Q)
\end{aligned}
$$

where the last term is negative from (A.1) and the two terms in brackets are negative from (B.1). Hence $d^{2} \Pi(Q, Q) / d Q^{2}<0$, and thus $d \Pi(Q, Q) / d Q<0$ for $Q \geq Q^{* *}$.

- Step 3. For any fixed level of aggregate output $Q_{A}+Q_{B}=2 Q$, the aggregate profit $\Pi\left(Q_{A}, Q_{B}\right)=\left[P\left(Q_{A}, Q_{B}\right)-c\right] Q_{A}+\left[P\left(Q_{B}, Q_{A}\right)-c\right] Q_{B}$ is maximal for $Q_{A}=Q_{B}=Q$.

Let us fix the total output $Q_{A}+Q_{B}=2 Q$, and consider the impact of a variation in $Q_{i}$ (thus compensated by a mirror variation in $Q_{j}$, for $i \neq j \in\{A, B\}$ ). The aggregate profit being symmetric in $Q_{A}$ and $Q_{B}$, its derivative with respect to $Q_{i}$, holding $Q_{A}+Q_{B}$ fixed, can be expressed as

$$
\left.\frac{d \Pi\left(Q_{A}, Q_{B}\right)}{d Q_{i}}\right|_{Q_{A}+Q_{B}=2 Q}=\Psi\left(Q_{i}, Q_{j}\right)-\Psi\left(Q_{j}, Q_{i}\right)
$$

where

$$
\Psi\left(Q_{i}, Q_{j}\right) \equiv \frac{\partial \Pi\left(Q_{i}, Q_{j}\right)}{\partial Q_{i}}=P\left(Q_{i}, Q_{j}\right)-c+Q_{i} \partial_{1} P\left(Q_{i}, Q_{j}\right)+Q_{j} \partial_{2} P\left(Q_{j}, Q_{i}\right) .
$$


The RHS of (23) is equal to zero when $Q_{A}=Q_{B}=Q$; we now show that it is never positive when $Q_{i}>Q_{j}$. To see this, consider the derivative of $\Psi$ with respect to $Q_{i}$, holding $Q_{A}+Q_{B}$ fixed:

$$
\begin{aligned}
\left.\frac{d \Psi\left(Q_{i}, Q_{j}\right)}{d Q_{i}}\right|_{Q_{A}+Q_{B}=2 Q}= & \frac{\partial \Psi\left(Q_{i}, Q_{j}\right)}{\partial Q_{i}}-\frac{\partial \Psi\left(Q_{i}, Q_{j}\right)}{\partial Q_{j}} \\
= & 2 \partial_{1} P\left(Q_{i}, Q_{j}\right)+Q_{i} \partial_{11}^{2} P\left(Q_{i}, Q_{j}\right)+Q_{j}^{2} \partial_{12}^{2} P\left(Q_{j}, Q_{i}\right) \\
& -\left[\partial_{2} P\left(Q_{i}, Q_{j}\right)+\partial_{2} P\left(Q_{j}, Q_{i}\right)+Q_{i} \partial_{12}^{2} P\left(Q_{i}, Q_{j}\right)+Q_{j} \partial_{12}^{2} P\left(Q_{j}, Q_{i}\right)\right] \\
\leq & 0,
\end{aligned}
$$

where the inequality follows from (B.2a). Hence, if $Q_{i}>Q=\frac{Q_{A}+Q_{B}}{2}>Q_{j}$, then $\Psi\left(Q_{i}, Q_{j}\right) \leq$ $\Psi(Q, Q) \leq \Psi\left(Q_{j}, Q_{i}\right)$, implying that $(23)$ is never positive; it follows that, keeping total output $Q_{A}+Q_{B}=2 Q$ constant, the aggregate profit $\Pi\left(Q_{A}, Q_{B}\right)$ is maximal for $Q_{A}=Q_{B}=Q$.

Steps 2 and 3 together imply that $\Pi\left(Q_{A}, Q_{B}\right)<\Pi\left(Q^{* *}, Q^{* *}\right)$ whenever $Q_{A}+Q_{B}>2 Q^{* *}$; property $(\boldsymbol{\Gamma} .2)$ then follows from step 1.

Finally, we consider property $(\boldsymbol{\Gamma} .3)$, and assume that $($ A.1 $)-($ A.2 $)$ and $($ B.1 $)-($ B.3 $)$ hold.

By symmetry, it suffices to show that, say, $\partial \tilde{Q}_{B} / \partial \hat{q}_{B 1} \leq 1$. This is obvious when $\tilde{q}_{B 2}=0$, as then $\tilde{Q}_{B}=\hat{q}_{B 1}$. Consider now the case where $\tilde{q}_{B 2}>0$ which, as already noted, implies $P\left(\tilde{Q}_{B}, \tilde{Q}_{A}\right) \geq c>0$. If $\tilde{q}_{A 1}=0$, so that $\tilde{Q}_{A}=\hat{q}_{A 2}$, then from $(21)$ :

$$
\frac{\partial \tilde{Q}_{B}}{\partial \hat{q}_{B 1}}=\frac{\partial_{1} P\left(\tilde{Q}_{B}, \tilde{Q}_{A}\right)}{\tilde{\lambda}_{B}} .
$$

Assumptions (A.1) and (B.3a) together imply $\tilde{\lambda}_{B}<\partial_{1} P\left(\tilde{Q}_{B}, \tilde{Q}_{A}\right)<0$, and thus $\partial \tilde{Q}_{B} / \partial \hat{q}_{B 1}<$ 1.

When instead $\tilde{q}_{A 1}>0$, then from $(22)$ :

$$
\frac{\partial \tilde{Q}_{B}}{\partial \hat{q}_{B 1}}=\frac{\tilde{\lambda}_{A} \partial_{1} P\left(\tilde{Q}_{B}, \tilde{Q}_{A}\right)+\tilde{\mu}_{B A} \partial_{2} P\left(\tilde{Q}_{B}, \tilde{Q}_{A}\right)}{D},
$$

where $D>0$ under Assumption (B.1). It follows that this expression is less than one if and only if $D>\tilde{\lambda}_{A} \partial_{1} P\left(\tilde{Q}_{B}, \tilde{Q}_{A}\right)+\tilde{\mu}_{B A} \partial_{2} P\left(\tilde{Q}_{B}, \tilde{Q}_{A}\right)$; this amounts to:

$$
\begin{aligned}
& {\left[\partial_{2} P_{A}+\partial_{2} P_{B}+\left(\tilde{Q}_{A}-\hat{q}_{A 2}\right) \partial_{12}^{2} P_{A}+\hat{q}_{B 1} \partial_{12}^{2} P_{B}\right] } \\
\times & \left.\times \partial_{2} P_{B}+\left(\tilde{Q}_{B}-\hat{q}_{B 1}\right) \partial_{12}^{2} P_{B}+\hat{q}_{A 2} \partial_{12}^{2} P_{A}\right] \\
< & {\left[2 \partial_{1} P_{A}+\left(\tilde{Q}_{A}-\hat{q}_{A 2}\right) \partial_{11}^{2} P_{A}+\hat{q}_{B 1} \partial_{22}^{2} P_{B}\right] } \\
& \times\left[\partial_{1} P_{B}+\left(\tilde{Q}_{B}-\hat{q}_{B 1}\right) \partial_{11}^{2} P_{B}+\hat{q}_{A 2} \partial_{22}^{2} P_{A}\right],
\end{aligned}
$$

which holds under Assumption (B.3b) (for $(i, j)=(A, B)$ and $\left.\left(q_{i}, q_{j}\right)=\left(\hat{q}_{A 2}, \hat{q}_{B 1}\right)\right)$. 